\title{
Grundlegende Untersuchungen zum laminar-turbulenten Umschlag an der konvex gekrümmten Oberseite eines schiebenden Tragflügels
}

\author{
Dissertation \\ zur Erlangung des Doktorgrades \\ der Mathematisch-Naturwissenschaftlichen Fakultäten \\ der Georg-August-Universität Göttingen
}

\author{
vorgelegt von \\ Andreas Pöthke \\ aus \\ Bad Saarow / Pieskow
}

Göttingen 1999 
D 7

Referent: Prof. Dr. H. Eckelmann

Korreferent: Prof. Dr. D. Ronneberger

Tag der mündlichen Prüfung: 


\section{Inhaltsverzeichnis}

1 Einleitung $\quad 1$

1.1 Der Gegenstand der Untersuchungen . . . . . . . . . . . . . 2

1.2 Der Stand der Forschung . . . . . . . . . . . . . . . 3

1.3 Ziel der Arbeit .......................... 7

2 Experimenteller Aufbau $\quad 9$

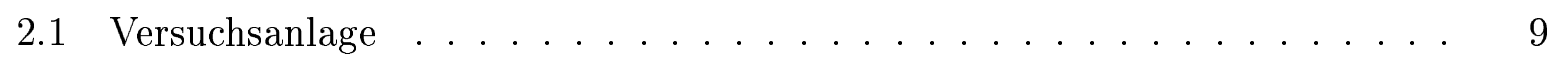

2.1.1 Anströmungsbedingungen . . . . . . . . . . . . 10

2.1.2 Das untersuchte Modell . . . . . . . . . . . 12

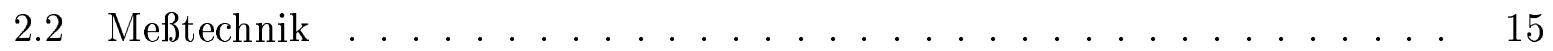

2.2.1 Hitzdrahtmessungen mit V-Sonden in stationären und instationären Geschwindigkeitsfeldern .............. 18

2.2.2 Bestimmung der Wellenzahlvektoren instationärer Instabilitätswellen 19

3 Grundströmungsentwicklung $\quad 23$

3.1 Notationen . . . . . . . . . . . . . . . . . . 23

3.1 Die verwendeten Koordinatensysteme . . . . . . . . . 23

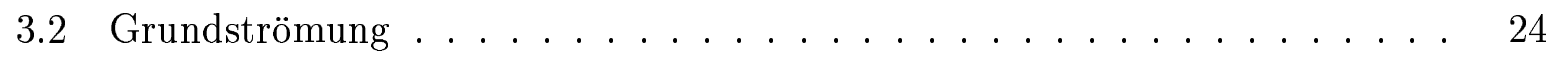

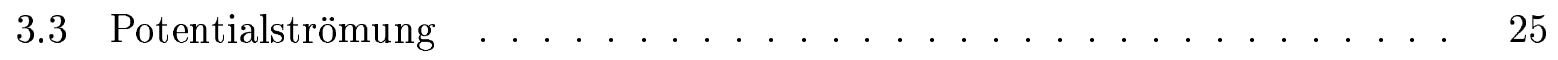

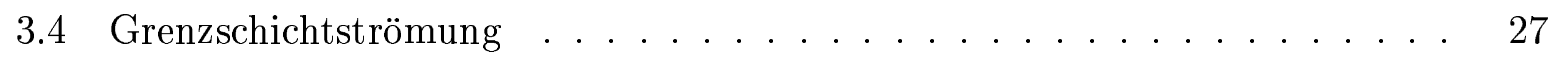

4 Lineare Störungsentwicklung in der Grenzschicht 33

4.1 Die lineare Stabilitätsanalyse . . . . . . . . . . . . . . . 34

4.2 Begrifflichkeiten . . . . . . . . . . . . . . . 37

4.3 Die Entwicklung ortsfester Wirbel . . . . . . . . . . . . . 37 
4.3.1 Identifikation stationärer Störungen . . . . . . . . . . 37

4.3.2 Präparation eines Feldes homogener stationärer Störungen . . . . 39

4.3.3 Stromabentwicklung verschiedener stationärer Störungen . . . . . . 40

4.3.4 Entwicklung einer einzelnen dominanten Störung und der Einfluß der Oberflächenkrümmung . . . . . . . . . . . 45

4.4 Die Entwicklung instationärer Störungen . . . . . . . . . . . . 46

4.4.1 Identifikation instationärer Störungen . . . . . . . . . . . . 46

4.4 .2 Stromabentwicklung instationärer Störungen . . . . . . . . . . . 52

5 Nichtlineare Entwicklung der Grenzschicht $\quad 59$

5.1 Die Grundströmungsverformung . . . . . . . . . . . . . . 60

5.2 Wechselwirkungen stationärer und instationärer Störungen . . . . . . . 63

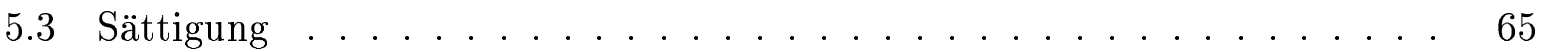

6 Zusammenbruch der laminaren Grenzschichtströmung $\quad 67$

6.1 Identifikation des Umschlags . . . . . . . . . . . . . . 68

6.2 Ablauf des Umschlags . . . . . . . . . . . . . . 69

6.3 Umschlag im Bereich eines negativen Druckgradienten . . . . . . . . 70

6.4 Umschlag im Bereich eines positiven Druckgradienten . . . . . . . . . 76

6.4.1 Hochfrequente Instabilitäten . . . . . . . . . . . 77

7 Zusammenfassung und Ausblick $\quad 85$

$\begin{array}{lr}\text { Literaturverzeichnis } & 89\end{array}$

$\begin{array}{lr}\text { Anhang } & 97\end{array}$

$\begin{array}{ll}\text { A Lineare Stabilitätsanalyse } & 97\end{array}$

$\begin{array}{ll}\text { B Profilkoordinaten HQ26 } & 101\end{array}$

$\begin{array}{ll}\text { C Druckverteilung } & 105\end{array}$

$\begin{array}{ll}\text { Danksagung } & 109\end{array}$ 


\section{Abbildungsverzeichnis}

2.1 Schematische Aufsicht auf den Niedergeschwindigkeitswindkanal Braunschweig 10

2.2 Leistungspektren des leeren Kanals bei $U_{\infty}=21 \mathrm{~m} / \mathrm{s}$, nach König [38] . . 11

2.3 Kohärenzfunktion des leeren Kanals bei $Q_{e}=21 \mathrm{~m} / \mathrm{s}$ über verschiedene spannweitige Entfernungen dy, nach König [38] . . . . . . . . . 11

2.4 Foto der Meßstrecke mit eingebautem Modell und Meßstativen . . . . . . . 13

2.5 Querschnitt des Tragflügelprofils HQ26; $d / c=14,8 \% \ldots \ldots \ldots \ldots$

2.6 Schematische Draufsicht auf das in den Kanal montierte Modell . . . . . 14

2.7 Blockdiagramm der Meßwertaufnahme im Experiment $\ldots \ldots \ldots$

2.8 Prinzipskizze einer Hitzdraht-V-Sonde $\ldots \ldots \ldots \ldots \ldots$

2.9 Meßprinzipskizze zur Bestimmung der Wellenzahlvektoren von Instabilitätswellen . . . . . . . . . . . . . . . . . 20

3.1 Skizze der verwendeten Koordinatensysteme . . . . . . . . . . . . . 23

3.2 Druckverteilung um das untersuchte Tragflügelprofil HQ26, $U_{\infty}=28 \mathrm{~m} / \mathrm{s}$,

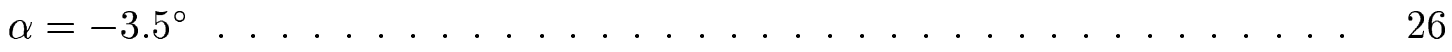

3.3 Lokaler Antrömwinkel am Grenzschichtrand der Profiloberseite . . . . . . . 27

3.4 Entwicklung von $\delta_{99,9}$ in Abhängigkeit der Profiltiefe für $U_{\infty}=28 \mathrm{~m} / \mathrm{s}$ aus Grenzschichtrechnungen . . . . . . . . . . . 28

3.5 Entwicklung von $\delta_{1}$ und $\delta_{2}$ in Abhängigkeit der Profiltiefe für $U_{\infty}=28 \mathrm{~m} / \mathrm{s}$ aus Grenzschichtrechnungen (Linien) und den Experimenten (Symbole),

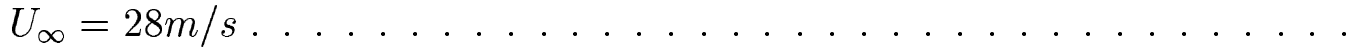

3.6 Vergleich der gemessenen, zeitlich und spannweitig gemittelten Strömung ( $<U_{s}>$ und $\left\langle V_{s}>\right.$ ) (Symbole) mit der aus der Druckverteilung numerisch rekonstruierten Grenzschichtströmung (Linie) für verschiedene Profiltiefenpositionen. $\left(U_{\infty}=28 \mathrm{~m} / \mathrm{s}, \Delta\right.$ : Wandabstandskorrektur.) $\ldots \ldots \ldots$ 
4.1 Isotachen der stationären Geschwindigkeitsfluktuationen $\tilde{U}_{s} / Q_{e}, \tilde{V}_{s} / Q_{e}$. Linienabstand $1 \%$, durchgezogene Linien entsprechen Geschwindigkeiten $\leq 0$, $x / c=0,326, \delta_{99,9}=1,65 \mathrm{~mm} \ldots \ldots \ldots \ldots$

4.2 Ermittlung der Wellenzahlen und der zugehörigen Amplituden stationärer Störungen, links: Spannweitige Variation der mittleren Geschwindigkeit $U_{s}$ in der Grenzschicht bei $z_{0}=0,5 \mathrm{~mm}, x_{c} / c=0,4, Q_{e}=44 \mathrm{~m} / \mathrm{s}$, rechts: Fouriertransformierte, geglättet. . . . . . . . . . . . .

4.3 Beeinflussung der spannweitigen Wellenlänge durch spannweitig periodische Rauhigkeitselemente bei $x / c=0,05, U_{\infty}=28 \mathrm{~m} / \mathrm{s}$, Meßort bei $x / c=0,217$

4.4 Interaktion spannweitig periodischer Rauhigkeitselemente mit Profilinhomogenitäten, $U_{\infty}=28 \mathrm{~m} / \mathrm{s}$, Meßort bei $x / c=0,217 \ldots \ldots$. . . .

4.5 Vergleich der Anregung jedes stationären Wirbels mit der Anregung jeden zweiten Wirbels, $U_{\infty}=28 \mathrm{~m} / \mathrm{s}$, Meßort bei $x / c=0,217 \ldots \ldots$

4.6 Stimulierung verschiedener spannweitiger Wellenlängen für den Meßort $x / c=0,261, U_{\infty}=28 \mathrm{~m} / \mathrm{s} \ldots \ldots \ldots \ldots$

4.7 Entwicklung stationärer Störungen verschiedener spannweitiger Wellenlänge gemäß linearer Instabilitätsanalyse, $U_{\infty}=28 \mathrm{~m} / \mathrm{s}$, Rechnungen v. Hein [46] mit linearem lokalem Verfahren unter Berücksichtigung von Oberflächen-

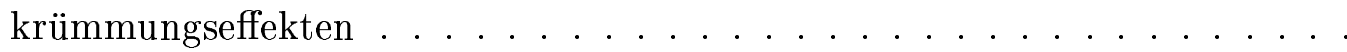

4.8 Experimentell ermittelte Stromabentwicklung der stationären Querströmungswirbel verschiedener spannweitiger Wellenlängen . . . . . . . .

4.9 Vergleich von numerischer und experimentell ermittelter relativer Störungsamplitudenentwicklung für stationäre Störkomponenten $\tilde{U}_{s}$ der Querwellenlänge $\lambda_{y_{c}}=6 \mathrm{~mm}$. Berechnungen von Hein [46] mit linearem NO$\mathrm{LOT} / \mathrm{PSE}[57] \ldots \ldots \ldots \ldots \ldots$

4.10 Betrag des normierten, spannweitig gemittelten Amplitudenspektrums von $u_{s}$ und $v_{s}$ bei $x_{c} / c=0,2166, Q_{e}=34,1 \mathrm{~m} / \mathrm{s}, U_{\infty}=28 \mathrm{~m} / \mathrm{s}$ und $\mathrm{z}=z_{\text {max-crossflow }}$

4.11 Experimentell bestimmter Betrag der Überlagerung aller Störungen zu einer Frequenz von $f=350 \mathrm{~Hz}$ für verschiedene Spannweitenpositionen. $Q_{e}=$ $31 \mathrm{~m} / \mathrm{s}, U_{\infty}=28 \mathrm{~m} / \mathrm{s} \ldots \ldots \ldots \ldots \ldots$

4.12 Schematische Darstellung der zur Kohärenzuntersuchung verwendeten Sonde. Die Abstandpfeile bezeichnen die gleichzeitig meßbaren Abstände. . . . 50

4.13 Kohärenzfunktion von $u_{s}$ für verschiedene spannweitige Abstände. $x / c=0,3 \ldots \ldots \ldots \ldots \ldots$

4.14 Gemessene Wellenlänge und Ausbreitungsrichtung der instationärer Instabilitäten im Vergleich mit Ergebnissen linearer Stabilitätsanalysen. $\left(x / c=0,3, U_{\infty}=28 \mathrm{~m} / \mathrm{s}\right)$ (gestrichelte Linien) ......... 
4.15 Isolinien konstanter relativer Störamplitude $\ln \left(u_{s} / u_{0}\right)$ in Abhängigkeit von Wellenzahl, Profiltiefe und Frequenz. Gestrichelt: Wellenzahl mit größter integraler Anfachung zu vorgegebener Frequenz. Ergebnisse numerischer Instabilitätsanalysen nach linearer lokaler Theorie von Hein [22]. $U_{\infty}=28 \mathrm{~m} / \mathrm{s}$

4.16 Isolinien konstanter Anfachung in der Nähe der neutralen Linie entsprechend lokaler linearer Theorie . . . . . . . . . . . . . . 54

4.17 Isolinien konstanter Anfachung in Abhängigkeit von Wellenzahl, Profiltiefe und Frequenz. Ergebnisse numerischer Instabilitätsanalysen nach linearer lokaler Theorie von Hein $[22] . U_{\infty}=28 \mathrm{~m} / \mathrm{s} \ldots \ldots \ldots$. . . . .

4.18 Experimentell ermittelte Entwicklung der relativen $v_{s}$-Amplitude über der Profiltiefenposition und der Frequenz. $U_{\infty}=28 \mathrm{~m} / \mathrm{s} \ldots \ldots$

4.19 Vergleich von numerischer und experimentell ermittelter relativer Störungsamplitudenentwicklung $u_{s} / u_{(s, 0)}$ für Störkomponenten der Querwellenzahl $\beta=1049 / m$ und den Frequenzen $f=400 H z, f=700 H z . \ldots . . . . \quad 57$

5.1 Entwicklung der $U_{s}$-Hauptströmungsstörung für die Profiltiefen $x / c=$ $0,115,0,220,0,310,0,425 \ldots \ldots \ldots \ldots$

5.2 Isolinien der normierten Querströmungs-RMS-Werte für verschiedene Profiltiefenpositionen $x / c=0,1455,0,261,0,326,0,377,0,411,0,437 \quad$. . .

6.1 Lage der Profiltiefenpositionen der Umschlagsuntersuchungen bezüglich der entdimensionalisierten Druckverteilung der Profiloberseite. . . . . . . .

6.2 Entwicklung des Betrages der normierten Amplitudenspektren von $\left(U_{s}, V_{s}\right)$ während der Transition bei $x / c=0,35, z_{0}=z_{\max C F} \ldots \ldots \ldots$

6.3 Entwicklung des Kreuzspektrums zwischen $U_{1, \text { eff }}$ und $U_{2, e f f},(\Delta s=1 \mathrm{~mm})$ während der Transition bei $x / c=0,35, z_{0}=z_{\max C F} \ldots \ldots \ldots$

6.4 Entwicklung des RMS-Wertes von $U_{s}$ während der Transition bei

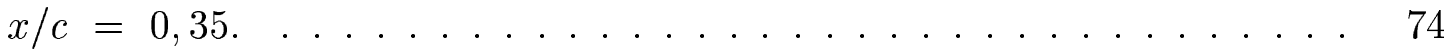

$6.5<\bar{U}_{s}>$ sowie Wendepunktsprofil (Links), Isolinien der stationären spannweitigen Modulation der spannweitig und zeitlich gemittelten Geschwindigkeitsprofile (Mitte) und der korrespondierenden RMS-Wert- Konturen für verschiedene Grenzschichtrandgeschwindigkeiten an der Position $x / c=0,35$ (Rechts). Linienabstand der Konturdiagramme: $1 \% Q_{e}$. . . . . . .

6.6 Geschwindigkeitsverlauf $U_{s}$ und Betrag des Amplitudenspektrums in der Umgebung einer hochfrequenten sekundären Instabilität. . . . . . . . . 77

6.7 Effektive Kühlgeschwindigkeiten der beiden Einzelhitzdrähte in der Umgebung hochfrequenter Geschwindigkeitsfluktuationen. . . . . . . . . . 
6.8 Gestalt der zeitl. gemittelten Grenzschicht und der RMS-Werte bei $x_{c} / c=$ $0,425, U_{\infty}=28 \mathrm{~m} / \mathrm{s}$. Die Kreise bezeichnen das Zentrum der maximalen Häufigkeit hochfrequenter Störungen. . . . . . . . . . . . .

6.9 Zusammenhang von $U_{s}, U_{r m s}$ und der Anzahl hochfrequenter Geschwindigkeitsschwankungen über einen Zeitraum von 2 Sekunden $\left(x_{c} / c=0,425\right.$, $z=0,56 m m) . \ldots \ldots \ldots \ldots$ 


\section{Tabellenverzeichnis}

2.1 Charakteristika des Niedergeschwindigkeitswindkanals Braunschweig . . . 9

2.2 Integrale Werte der Turbulenz des NWB bei geschlossener Meßstrecke normiert auf $U_{\infty}=21 \mathrm{~m} / \mathrm{s} \ldots \ldots \ldots \ldots \ldots . \ldots \ldots 12$

2.3 Geometriedaten des untersuchten Modells . . . . . . . . . . 12

4.1 Parameter der am meisten angefachten Wellen gemäß linearer lokaler Theorie 54

4.2 Parameter der Wellen mit der größten integralen Anfachung gemäß linearer lokaler Theorie . . . . . . . . . . . . . . 56

5.1 Vergleich der gemessenen Sättigungsamplituden zwischen dem DLR Prinzipexperiment „Schiebende Platte“ und dem konvex gekrümmten Tragflügelprofil HQ26. Alle Angaben in Prozent der Außenströmungsgschwindigkeit. Die Werte in Klammern wurden unter Verwendung von periodischen Rauhigkeitselementen gemessen. . . . . . . . . . . . . .

6.1 Maximale rel. Amplituden der stationären spannweitigen Geschwindigkeitsmodulation und der RMS-Werte von $U s$ für verschiedene Grenzschichtrandgeschwindigkeiten. . . . . . . . . . . . . . 72

6.2 Eigenschaften beobachteter hochfrequenter Geschwindigkeitsfluktuationen. $\quad 78$

B.1 Profilkoordinaten Oberseite, $\mathrm{c}=0,65 \mathrm{~m} \ldots \ldots . \ldots . \ldots 2$

B.2 Profilkoordinaten Unterseite, $\mathrm{c}=0,65 \mathrm{~m} \ldots \ldots . \ldots . \ldots$

C.1 Druckverteilung Unterseite . . . . . . . . . . . . 106

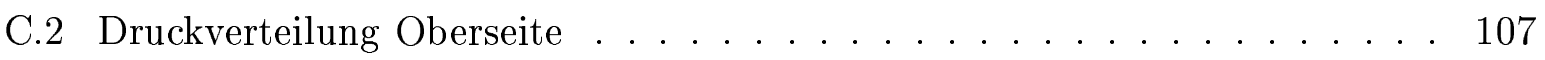




\section{Bezeichnungen}

\section{Lateinische Buchstaben}

$\begin{array}{ll}\text { A } & \text { Kalibrierungskonstante Kingsches Gesetz } \\ \mathrm{B} & \text { Kalibrierungskonstante Kingsches Gesetz } \\ \mathrm{c} & \text { Flügeltiefe } c=0,65 m \\ \text { c.c. } & \text { konjugiert-komplex } \\ c_{g r} & \text { Gruppengeschwindigkeit, } c_{g r}=d \omega / d k \\ c_{p} & \text { Druckbeiwert, } c_{p}=\left(p_{w}-p_{\infty}\right) /\left(\frac{\rho}{2} U_{\infty}^{2}\right) \\ c_{p h} & \text { Phasengeschwindigkeit, } c_{p h}=\omega / k \\ f & \text { Frequenz } \\ \vec{k}=(\alpha, \beta) & \text { Wellenvektor, }|k|=2 \pi / \lambda \\ \mathrm{Ma} & \text { Machzahl } \\ \mathrm{n} & \text { Kalibrierungskonstante Kingsches Gesetz } \\ \mathrm{n}, \mathrm{m}, \mathrm{l}, \mathrm{k} & \text { beliebige ganze Zahlen } \\ p_{\infty} & \text { statischer Druck der Anströmung } \\ p_{w} & \text { Wanddruck } \\ Q & \text { Wärmemenge } \\ Q_{e} & \text { Betrag der lokalen Geschwindigkeit am Grenzschichtrand } \\ U_{\infty} & \text { Anströmgeschwindigkeit } \\ \vec{q}=(u, v, w) & \text { momentaner lokaler Geschwindikeitsvektor } \\ R_{z} & \text { lokale, gemittelte Rauhtiefe nach DIN 4768/1 } \\ \mathrm{t} & \text { Zeit } \\ \mathrm{Tu} & \text { Turbulenzgrad der Anströmung } \\ \vec{U}(x, y, z, t) & \text { Tu=1/U⿻ } \sqrt{1 / 3\left(\overline{u^{2}}+\overline{v^{2}}+\overline{w^{2}}\right)} \\ \overline{\vec{U}}(x, y, z) & \text { Geschwindigkeitsvektor am Ort }(\mathrm{x}, \mathrm{y}, \mathrm{z}) \text { zur Zeit t } \\ =(U, V, W)(x, y, z) & \text { am Ort }(\mathrm{x}, \mathrm{y}, \mathrm{z}) \\ \vec{u} & \text { Schwankungsgrößen der lokalen Geschwindigkeit } \\ =(u, v, w)(x, y, z, t) & \text { am Ort }(\mathrm{x}, \mathrm{y}, \mathrm{z}) \text { zur Zeit t },(u, v, w)(x, y, z)=\vec{U}-(U, V, W) \\ <\vec{U}(x, z)> & \text { in y-Richtung gemitteltes Geschwindigkeitsprofil. } \\ (x, y, z) & \text { Koordinatensystem des Kanals, x:stromab } \\ & \end{array}$




$\begin{array}{ll}\left(x_{c}, y_{c}, z\right) & \text { Koordinatensystem des Models, vorderkantenparallel } \\ x_{c} & \text { Profiltiefenrichtung } \\ y_{c} & \text { Spannweitenrichtung } \\ z_{c} & \text { wandnormale Richtung } \\ \left(x_{s}, y_{s}, z\right) & \text { Stromlinienkoordinatensystem, lokal } \\ & x_{s} \text { Stromabrichtung } \\ \lambda_{y_{(c, s t)}} & \text { spannweitig stimulierte Wellenlänge }\end{array}$

\section{Griechische Buchstaben}

$\begin{array}{ll}\alpha & \text { Anstellwinkel des Modells } \\ \alpha, \beta & \text { komplexe wandparallele Wellenzahlkomponenten } \\ \gamma & \text { Winkel von } \vec{k} \\ \gamma_{I} & \text { Intermittenzfaktor } \\ \delta, \delta_{99,9} & \text { lokale Grenzschichtdicke } \\ \delta_{1} & \text { lokale Verdrängungsdicke } \delta_{1}:=\int_{0}^{\infty}\left(1-U_{s} / U_{s, e}\right) d z \\ \delta_{2} & \text { lokale Impulsverlustdicke } \delta_{2}:=\int_{0}^{\infty} U_{s} / U_{s, e}\left(1-U_{s} / U_{s, e}\right) d z \\ \lambda & \text { Wellenlänge } \\ \nu & \text { kinematische Zähigkeit } \\ \nabla & \text { Differentialoperator } \\ \rho & \text { Dichte der Luft } \\ \phi_{\infty} & \text { geometrischer Schiebewinkel } \\ \phi_{e} & \text { lokaler Anströmwinkel am Grenzschichtrand } \\ \omega & \text { Kreisfrequenz, } \omega=2 \pi f\end{array}$

\section{Formelsymbole}

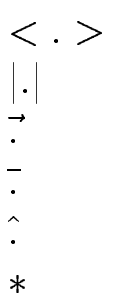

\section{Indizes}

$c$

e spannweitige Mittelung

Absolutbetrag

vekorielle Größe

zeitliche Mittelung

Amplitude

konjugiert komplexe Größe körperorientierts Koordinatensystem

Grenzschichtrand 
mess

num

rms

$s$

sek

$w$

$W P$ experimentell ermittelter Wert

berechneter Wert

Effektivwert (root-mean-square value)

am Stromlinienkoordinatensystem orientierte Größe

sekundär

Wand

Wendepunkt 


\section{Kapitel 1}

\section{Einleitung}

In Natur und Technik unterscheidet man zwei Strömungsformen, die geschichtete, in geordneten Bahnen verlaufende (laminare) und die turbulente Strömung. Beiden Formen lassen sich charakteristische Eigenschaften zuordnen.

Die laminare Strömungsform zeichnet sich durch eine geringe Energiedissipation aus. Die turbulente Strömungsform ist gekennzeichnet durch eine starke Energiedissipation. Kinetische Energie aus der Bewegung des Fluids wird über eine Kaskade von großen zu kleinen Längenskalen umgewandelt in Wärme, mechanische Arbeit oder auch chemisch gebunden. Vermittelt wird diese Umwandlung durch die Zähigkeit. Eine ständige Zufuhr von Energie ist nötig, die Strömung in turbulenter Bewegung zu halten.

Neben dem Reibungswiderstand unterscheiden sich laminare und turbulente Strömungen auch im Ablöseverhalten, dem Wärme- und Stoffaustausch sowie in Vermischungsprozessen. Darüber hinaus können Ergebnisse von Navier- Stokes Rechnungen nicht besser sein als die Vorgabe des Umschlagsortes. Aus diesen Gründen ist das Transitionsproblem von hoher Bedeutung in der Technik. Seit der Veröffentlichung der Beobachtungen von Reynolds [1] im Jahre 1883 ist es Gegenstand zahlreicher wissenschaftlicher Arbeiten in verschiedenen Anwendungsbereichen, insbesondere im Flugzeugbau.

Reibungswiderstand und Ablösung bestimmen neben dem Seitenverhältnis der Tragflügel die aerodynamische Güte von Flugzeugen. Wegen des hohen Treibstoffverbrauchs moderner Verkehrsflugzeuge ${ }^{1}$ wurden während der letzten beiden Jahrzehnte von den großen Verkehrsfluggesellschaften große Anstrengungen unternommen, die Grenzschicht von Tragflügeln, Rumpf und Leitwerken durch besondere Vorkehrungen bei der Auslegung oder durch besondere Maßnahmen wie partielle Grenzschichtabsaugung, Tilgung bzw. Abschwächung von Instabilitäten, die zum Zusammenbruch der laminaren Strömung führen, laminar zu halten. Das verlangt eine detaillierte Kenntnis der wirksamen Instabilitätsmechanismen. Die Bestimmung des Widerstands erfordert die präzisen Voraussage des Umschlagsortes.

\footnotetext{
${ }^{1}$ z.B. Airbus A320 ca. 5 Liter Kerosin pro $100 \mathrm{~km}$ und Passagier
} 
Der Gegenstand der vorliegenden experimentellen Untersuchungen liegt in diesem Bereich. Es sollen die physikalischen Kenntnisse geschaffen werden, die die optimale Auslegung eines schiebenden Flügels bezüglich der Stabilität der laminaren Strömung sowie der Maßnahmen zur künstlichen Grenzschichtstabilisierung ermöglichen.

Bei modernen Verkehrsflugzeugen wird die Tragflügelumströmung bereits im Nasenbereich instabil und gewöhnlich dort schon turbulent. Das bedeutet, daß die Tragflügelumströmung überwiegend turbulent ist. Die Laminarhaltung dieser Strömung birgt daher ein außerordentliches Sparpotential an Treibstoff. So ist es nicht verwunderlich, daß seit mehr als einem Jahrzehnt ein Schwerpunkt bei Entwicklung moderner Großverkehrsflugzeuge in der Nutzung dieses Sparpotentials liegt.

Das besondere Problem, dem sich dabei Entwurfsaerodynamiker gegenübergestellt sehen, ist die genaue Beurteilung der Stabilität bzw. des Instabilitätsverhaltens am schiebenden Flügel. Die gegenwärtig zur Verfügung stehenden Stabilitätsberechnungsverfahren, bzw. die auf ihnen beruhenden Kriterien, den laminar-turbulenten Übergang richtig vorherzusagen, reichen dazu nicht aus.

\subsection{Der Gegenstand der Untersuchungen}

Betrachtet man den Bereich der Tragflügelumströmung, so findet man die Entwicklung der Grenzschicht zahlreichen Einflüssen unterworfen.

An der Anströmungsseite kommt es zur Ausbildung einer Anlegelinie, die die Umströmung in Ober - und Unterseitenströmung teilt. Von ihr ausgehend, dickt sich die Wandgrenzschicht in Flügeltiefenrichtung langsam auf. Das Verdicken der Grenzschicht wird dabei zunächst gehemmt durch eine beschleunigte Strömung außerhalb der Grenzschicht in Folge des negativen Druckgradienten in Tragflügeltiefenrichtung. Im Bereich des Druckminimums und stromab von diesem wird die Wandgrenzschicht sehr schnell dicker. An diesen Stellen kann es leicht zur Ausbildung einer abgelösten Wandgrenzschicht kommen. Dabei bilden sich Rückstromgebiete über der Tragflügeloberfläche, die zu einem Zusammenbruch des Druckgradienten führen und den Luftwiderstand erhöhen.

Die sich ausbildende Wandgrenzschicht über einem unendlich langen, schiebend angeordneten Tragflügel ist dreidimensional: Wir betrachten im folgenden die Stromlinie am Grenzschichtrand. Der Schiebewinkel bewirkt im körperorientierten Bezugssystem eine für alle Profiltiefenpositionen konstante spannweitige Geschwindigkeitskomponente. Der Druckgradient in Profiltiefenrichtung ändert dagegen seine zugehörige Geschwindigkeitskomponente. Daraus resultiert eine Stromlinienkrümmung am Rande der Grenzschicht. Die lokale Beschleunigung eines Fluidelements richtet sich nach dem aktuellen Kräftegleichgewicht aus allen beteiligten Druckkräften und den Zentrifugalkräften als Resultat der Stromlinienkrümmung. Da die Grenzschicht in erster Näherung für Drucke transparent ist, die Zentrifugalkraft sich aber mit der Geschwindigkeit ändert, sind Zentrifugal- und Druckkräfte innerhalb der Grenzschicht nicht mehr im Gleichgewicht. Es bildet sich eine 
Ausgleichsströmung quer zur reibungsfreien Strömung außerhalb der Grenzschicht. Diese Ausgleichsströmung ändert die Strömungsrichtung im Inneren der Grenzschicht und beeinflußt deren Stabilitätseigenschaften. Es existieren Raumrichtungen, in denen die auf sie projizierten Geschwindigkeitsprofile Wendepunkte aufweisen. Nach Rayleigh (1880)[2] ist ein Wendepunkt in Geschwindigkeitsprofilen eine notwendige Bedingung für Instabilität und für Grenzschichtströmungen nach Tollmien (1935)[3] auch eine hinreichende Bedingung für Instabilität - vorbehaltlich einer Korrektur infolge des Zähigkeitseinflusses.

\subsection{Der Stand der Forschung}

Gray (1952)[4][5] bemerkte bei Freiflug- und Laborversuchen erstmals, daß die Grenzschicht schiebender Tragflügel viel früher turbulent wurde, als die der korrespondierenden nichtschiebenden Tragflügel. Das war zur damaligen Zeit eine große Überraschung, da bis dahin der Beschleunigung von Grenzschichtströmungen eine stabilisierende Wirkung nachgesagt wurde. Anstrichbilder zeigten stromab orientierte äquidistante Streifen im Vorfeld des Zusammenbruchs der laminaren Wandgrenzschichtströmung, die als Folge von stationären Wirbeln, einer Instabilitätsform dreidimensionaler Grenzschichten, interpretiert wurden. Aufgrund dieser Beobachtungen entwickelten Gregory et al. (1955) [6] ihre experimentellen und theoretischen Untersuchungen zur Stabilität und Transition der Strömung oberhalb einer rotierenden Scheibe (ebenfalls Wendepunktinstabilität als Folge einer Querströmungskomponente) und arbeiteten deren charakteristische Eigenschaften heraus.

Dreißig Jahre später, Mitte der achtziger Jahre, rückte die Querströmungsinstabilität durch Experimente von Poll (1985)[7] am RAE an einem schiebenden Zylinder, Arnal et al. (1984)[8] sowie Michel et al. (1985)[9] an einem schiebenden Tragflügel und Saric und Yeates (1985)[10] an einer schiebenden Platte mit aufgeprägtem Druckgradient wieder ins Blickfeld der Strömungsforschung. Alle bestätigten die Aussagen Stuarts aus [6] auch für die Querströmungsinstabilität in der beschleunigten Region der Umströmung schiebender Tragflügel.

Gegenwärtig laufen weltweit vier große Forschungsprogramme, die sich mit den Grundlagen zur Transition an schiebend angeordneten Tragflügeln beschäftigen:

- Saric et al. an der Arizona State University (ASU) in Tempe, USA, am schiebenden Tragflügel,

- Kachanov et al. am Institut für theoretische und angewandte Mechanik (ITAM) in Novosibirsk, Rußland, an der schiebenden Platte mit aufgeprägtem Druckgradient,

- Itoh et al. am National Aerospace Laboratory (NAL) in Tokio, Japan, am schiebenden Zylinder,

- Bippes et al. am Deutschen Zentrum für Luft- und Raumfahrt (DLR) in Göttingen, an Modellen mit ebenen, konvex und/oder konkav gewölbten Oberflächen. 
Eine ausführliche Zusammenfassung des Wissenstandes bis zum Jahr 1994 findet man bei Saric[11] und für die Zeit danach bei Bippes [37].

Grenzschichten mit Querströmung sind instabil gegen Störungen in Form ebener Wellen. Im Grenzfall der Frequenz Null bilden sich ortsfeste, in Stromlinienrichtung orientierte Wirbel wie die von Gray beobachteten. Für von Null verschiedene Frequenzen zeigt sich die Grenzschichtströmung gegen diejenigen Störungen besonders instabil, deren Wellenvektor in eine Richtung zeigt, in der das auf diese Richtung projizierte Geschwindigkeitsprofil einen Wendepunkt aufweist ${ }^{2}$. Eine detaillierte Abhandlung zum Problem linearer Stabilität in Grenzschichten findet man bei Mack (1984)[12].

Die Entwicklung von Störungen durchläuft zunächst einen linearen Bereich, in dem sich die vorhandenen Störungen, ob stationär oder instationär, unabhängig voneinander, also nebeneinander her, entwickeln.

In der Identifikation erwarteter Störungen in diesem Bereich bezüglich Wellenlänge, Phasenrichtung und Frequenz herrscht seitens der linearen Theorie gute Übereinstimmung mit den Experimenten (siehe u.a. [13],[15]). Das Wachstum der Störamplituden wird von ihr jedoch nicht zuverlässig richtig wiedergegeben (siehe z.B. Bippes (1991)[16]). Darüber hinaus wurde gezeigt[34], daß instationäre Störungen in der Anströmung (Turbulenzgrad) laufende Wellen hervorrufen, während die stationären Wirbel im wesentlichen durch Oberflächenrauhigkeiten angeregt werden.

Ein theoretischer Ansatz zur besseren Beschreibung des linearen Bereichs kam mit der Einführung der parabolisierten Stabilitätsgleichungen (PSE) durch Herbert und Bertolotti (1987) $[17]^{3}$. Inzwischen wird auch der nichtlineare Verlauf erfaßt.

Sie berücksichtigen nichtlokale Effekte (Grenzschichtaufdickung) sowie die Stromlinienkrümmung und zeigen sich damit der klassischen lokalen Stabilitätsanalyse insbesondere hinsichtlich der korrekten Vorhersage des Störungswachstums im linearen und vor allem im nichtlinearen Bereich überlegen (vergl. Lerche und Bippes (1995)[19], Haynes (1996)[20], Reibert und Saric (1997)[23]). Im Gegensatz zu den lokalen Stabilitätsgleichungen müssen neben den Rand- auch die Anfangsbedingungen vorgegeben werden. Damit kann die Größe der Amplituden bzw. deren Wachstum quantitativ richtig bestimmt werden.

Erreicht ein Teil des vorhandenen Störspektrums eine bestimmte Größe, beginnen die Störungen miteinander zu interagieren. Ab diesem Punkt sind die Voraussetzungen linearer Theorie nicht mehr erfüllt. Einerseits beginnen die stationären Störungen die vorhandene Grundströmung zu deformieren und damit den die Instabilität treibenden Mechanismus zu verändern, andererseits kommt es zu Wechselwirkungen zwischen stationären und instationären Störungen, die sich in Form von spannweitigen Modulationen der Amplituden der instationären Störungen manifestieren. Meßbare Auswirkungen dieser Prozesse sind Sättigungseffekte im Amplitudenwachstum, die Generierung neuer Störspektralanteile sowie die spannweitige Modulation der Amplituden instationärer Störungen.

\footnotetext{
${ }^{2}$ Diese Profile werden in der Literatur kurz „Richtungsprofile“ genannt.

${ }^{3}$ Eine beispielhafte detailierte Darstellung des PSE - Verfahrens für die Blasius-Grenzschicht und einen Vergleich mit DNS Rechnungen findet man bei Bertolotti et al.[18]).
} 
Zur Beschreibung der Wechselwirkungen stationärer und instationärer Störungen benutzte Reed (1989)[24] eine sekundäre Theorie, die das Auftreten von superharmonischen stationären Wirbeln vorhersagt, deren Anfachung die der primären stationären Wirbel um das Dreifache übertrifft. Fischer und Dallmann (1991)[25] beschrieben die spannweitigen Amplitudenmodulationen laufender Wellen mittels sekundärer Stabilitätsanalyse einer durch stationäre Wirbel deformierten Grundströmung. Sie fanden das Auftreten einer sekundären Instabilität bereits für sehr kleine Amplituden der primären stationären Wirbel. Die zugehörigen instationären Störungen besitzen Frequenzen, die nur geringfügig höher sind als die primär angefachten, und entziehen sich damit der Möglichkeit eines direkten experimentellen Nachweises.

Theoretische Annäherungen zur Beschreibung von nichtlinearen Entwicklungen wurden einerseits durch DNS-Rechnungen (Meier und Kleiser (1991)[26], Wagner (1992)[27], Wintergerste und Kleiser (1996)[28] sowie Müller et al. (1995)[29]) andererseits durch nichtlineare PSE-Rechnungen (Malik et al. (1994)[30], Bertolotti (1995)[31] und Haynes (1996)[20]) versuchten und zeigten qualitative Übereinstimmungen zu den experimentellen Befunden für die Abhängigkeiten von den Anfangsbedingungen. Quantitative Vergleiche offenbarten die wichtige Rolle der Anfangsbedingungen, die im wesentlichen durch Restturbulenzen der ankommenden Strömungen und Oberflächenrauhigkeiten modifiziert werden: In einer Umgebung mit vergleichsweise hohem Turbulenzgrad wurde die Anfachung und Sättigung der stationären Störungen nur dann richtig wiedergegeben, wenn neben der Rauhigkeit auch das anfängliche instationäre Störungsniveau in der Rechnung berücksichtigt wurde (Bertolotti[31]). Für niedrigturbulente Umgebungen wurde die Entwicklung stationärer Störungen auch unter Vernachlässigung instationärer Moden korrekt beschrieben (Haynes[20]).

Bei bisherigen Betrachtungen wurde die Wirkung der Oberflächenkrümmung nur am Rande behandelt. Erste Betrachtungen (Collier\&Malik[21]) sagten einen deutlich dämpfenden Effekt konvexer Krümmung voraus. Sie basierten jedoch auf einer mathematisch nicht konsistenten Näherung. Später wurden verbesserte Modelle eingeführt (Hein[22]), die allerdings u.a. immer noch Vernachlässigungen der metrischen Terme normal zur Wand enthalten, die nur gerechtfertigt sind, solange $\delta / R<O(1 / R e)$ gilt. ( $\delta$ bezeichnet die Grenzschichtdicke und $R$ den Krümmungsradius.) Diese Modelle offenbaren ebenso einen dämpfenden Effekt der konvexen Oberflächenkrümmung, der allerdings durch die oftmals mit der Krümmung verbundene Destabilisierung durch die Nichtparallelität der Strömung abgeschwächt wird. Beide Effekte haben formal die gleiche Größenordnung $O(1 / R e)$. Gerade im vorderen Bereich des Tragflügels, der für die Laminartechnologie und den laminarturbulenten Übergang von besonderer Bedeutung ist, werden die Grenzen der Anwendbarkeit der Verfahren erreicht und überschritten. Inwieweit daher die modernen PSE Verfahren den praktischen Anforderungen genügen, muß das Experiment zeigen.

Ein weiteres Problem für die Anwendung der PSE-Verfahren beim Entwurf von Laminarflügeln und für die Voraussage der Transition besteht im starken Anwachsen der Grenzschicht im Nasenbereich und in der mangelnden Kenntnis des Stabilitätsproblems der Anlegelinienströmung, sowohl das physikalische Verständnis betreffend, als auch die 
theoretische Formulierung. Daher sind experimentelle Untersuchungen des engeren Nasenbereichs, wo Anlegelinieninstabilität und Querströmungsinstabilität bei starker Nichtparallelität und starker konvexer Oberflächenkrümmung zusammenkommen, sehr schwer zu interpretieren. Solange keine zuverlässigen Stabilitätsberechnungsverfahren zur Verfügung stehen, ist es für den Entwurfsaerodynamiker sehr schwer, die Stabilität der Strömung zu optimieren bzw. über Notwendigkeit und Art zusätzlicher Maßnahmen zur Laminarhaltung der Tragflügelströmung zu entscheiden. Das führte zu der hier gestellten Aufgabe, den Effekt konvexer Oberflächenkrümmung auf Querströmungsinstabilität unbeeinflußt von der Anlegeinstabilität zu untersuchen und insbesondere Daten für die Modellierung von Stabilitätsberechnungsverfahren und die Validierung bestehender Verfahren zu liefern.

Die nächste Stufe des Transitionsprozesses stellt der Zusammenbruch der laminaren Grenzschichtströmung dar. In der durch stationäre Wirbel stark verformten Grenzschicht wurden bei Experimenten unmittelbar vor dem Zusammenbruch hochfrequente Störungen gefunden, deren Frequenzen um eine Größenordnung höher waren als die den primären Instabilitäten zugeordneten Störungen (Poll[7], Kohama et al.[33], Deyhle et al.[34], Nishioka et al.[35] für zweidimensionale Kanalströmung).

Diese Befunde bildeten die Grundlage für eine sekundäre Stabilitätsanalyse einer durch primäre stationäre Wirbel stark verformten Grenzschicht durch Fischer et al.[36] und Malik et al.[30]. Beide fanden eine starke sekundäre Instabilität gegen laufende Wellen mit Frequenzen, die um eine Größenordnung über denen der primären Instabilität lagen, in der Umgebung eines sich auf grund nichlinearer Störentwicklung ausbildenden zweiten Wendepunktes im oberen Drittel der Grenzschicht.

Lerche[14] bestätigte in seinem Experiment mit künstlich angeregten primären stationären und instationären Wellen diese Resultate und konnte darüber hinaus zeigen, daß nicht das stationäre, sondern das momentane Grenzschichtprofil über die Ausbildung eines Wendepunktes zuerst sekundär instabil wird. Durch die hohe Anfachung der sekundären Instabilität kommt es bereits kurz nach ihrem Auftreten zum Zusammenbruch der laminaren Grenzschichtströmung, wodurch sich diese Instabilität hervorragend für die Transitionsvorhersage eignen würde.

Gegenwärtig gibt es noch keine experimentell ermittelten Anfachungen, Ausbreitungsrichtungen und Wellenlängen für diese Instabilitätswellen. Auch sind die Auflösungen der DNSbzw. PSE-Rechnungen zu grob, um diese sekundären Instabilitäten zu erfassen. Ob für die Einleitung der Endphase der Transition diese hochfrequente sekundäre Instabilität der einzige Mechanismus ist, oder ob neben diesem Umschlagsmechanismus noch weitere existieren, ist gegenwärtig Gegenstand der Forschung.

Eine Übersicht über den gegenwärtigen Erkenntnisstand zur Querströmungsinstabilität inklusive einer Vielzahl von Vergleichen unterschiedlicher experimenteller und numerischer Resultate sowie einem umfangreichen Literaturverzeichnis wurde von Bippes in [37] erstellt. 


\subsection{Ziel der Arbeit}

Ziel der Arbeit ist es, durch Schaffung einer breiten experimentellen Datenbasis, an einem grundlegenden Experiment unter den Rechnungen möglichst gut zugänglichen Strömungsbedingungen, die Entwicklung von Instabilitätsberechnungsverfahren zu unterstützen und deren Ergebnisse zu validieren, um die Möglichkeit der Transitionsvorhersage zu verbessern. Ein besonderes Augenmerk liegt dabei auf dem Einfluß der konvexen Oberflächenkrümmung. 


\section{Kapitel 2}

\section{Experimenteller Aufbau}

\section{$2.1 \quad$ Versuchsanlage}

Alle Experimente wurden im Niedergeschwindigkeitswindkanal Braunschweig (NWB), einem Kanal aus dem Verbund der Deutsch - Niederländischen Windkanäle (DNW), durchgeführt. Bei ihm handelt es sich um einen kontinuierlich arbeitenden Windkanal mit einem Meßstreckenquerschnitt von 3,25 m x 2,8 m (vgl. Abbildung 2.1 auf Seite 10). Seine wichtigsten Daten sind in Tabelle 2.1 zusamengefaßt, wobei $R e_{\text {Kanal }}$ die mit der Wurzel aus dem Kanalquerschnitt gebildete, sogenannte Kanalreynoldszahl ist.

\begin{tabular}{|c|c|}
\hline Querschitt & $3,25 \mathrm{~m} \times 2,8 \mathrm{~m}$ \\
\hline Typ & offen, geschlossen \\
\hline Länge & $6,2 \mathrm{~m}$ \\
\hline Geschwindigkeit & $90 \mathrm{~m} / \mathrm{s}$ \\
\hline$R e_{\text {Kanal }}$ & $1,8 \cdot 10^{6}$ \\
\hline Kontraktionsverhältnis & 5,6 \\
\hline Antriebsleistung & $1,4 \cdot 10^{6} \mathrm{MW}$ \\
\hline Turbulenzgrad & $T u_{\text {Ges }}<0,08 \%$ \\
\hline
\end{tabular}

Tabelle 2.1: Charakteristika des Niedergeschwindigkeitswindkanals Braunschweig 


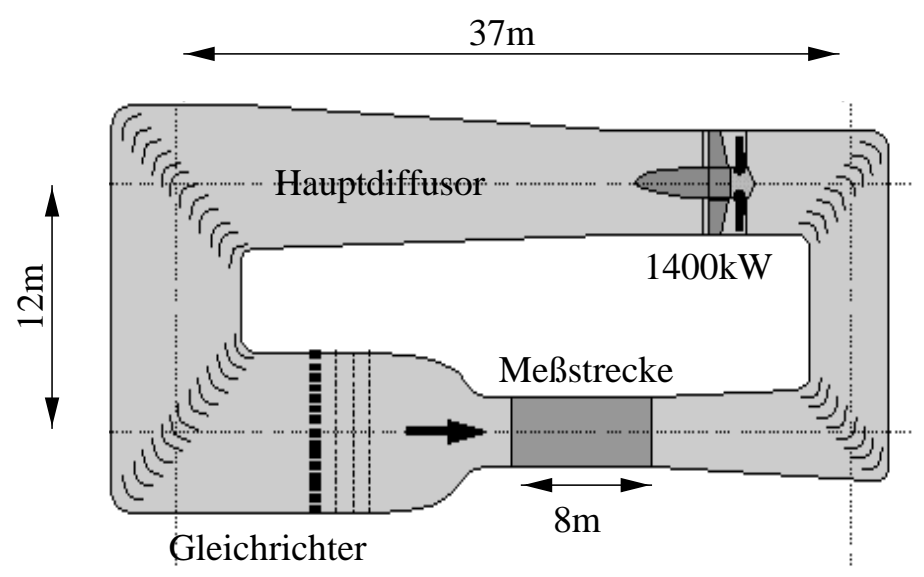

Abbildung 2.1: Schematische Aufsicht auf den Niedergeschwindigkeitswindkanal Braunschweig

\subsubsection{Anströmungsbedingungen}

Wie bereits in der Einleitung erwähnt, hängt die Anregung von Störungen, deren nichtlineare Entwicklung sowie die Transitionslage vom Störgehalt der Umgebung ab. In Windkanalversuchen stellen Anströmungsgeschwindigkeits- und Druckschwankungen die wesentliche Anregungsquelle instationärer Störungen dar. Da die Rezeptivität, das heißt das Einkoppeln der Störungen in die Grenzschicht und die Umwandlung in Instabilitäten für beide instationäre Störungsformen verschieden ist, ist die genaue Kenntnis dieser Umgebungsbedingungen von hoher Bedeutung für die Interpretation der Ergebnisse und den Vergleich mit der Theorie.

Bei der Beurteilung der Kanalturbulenz ist es zweckmäßig, zwischen der kurzskalig kohärenten Wirbelstärke und dem langskalig kohärenten akustischem Anteil der Kanalturbulenz zu unterscheiden. Der Gesamtturbulenzgrad des leeren Kanals

$$
T u_{G e s}=1 / U_{\infty} \sqrt{1 / 3\left(\overline{u^{2}}+\overline{v^{2}}+\overline{w^{2}}\right)}
$$

ist mit $T u_{G e s}<0,08 \%$ angegeben. Detaillierte Untersuchen zum Turbulenzgrad des leeren Kanals von König [38] bestätigen das. Die am leeren Kanal mit geschlossener Meßstrecke durchgeführten Untersuchungen erbrachten folgende Ergebnisse:

Die kanalinduzierten Störungen liegen im wesentlichen im niederfrequenten Bereich. Ein sich typischerweise ergebendes Paar von Störspektren ist in Abbildung 2.2 auf Seite 11 dargestellt.

Im Bereich kleiner Frequenzen $(f<20 H z)$ dominieren Schwankungen in Hauptströmungsrichtung. Diese Schwankungen besitzen über große Entfernungen kohärente Spektralanteile bei der Gebläsedrehzahl und einigen ganzzahligen Vielfachen bis hin zur Schaufelfrequenz und sind damit dem akustischen Teil der Kanalturbulenz zuzuordnen. 

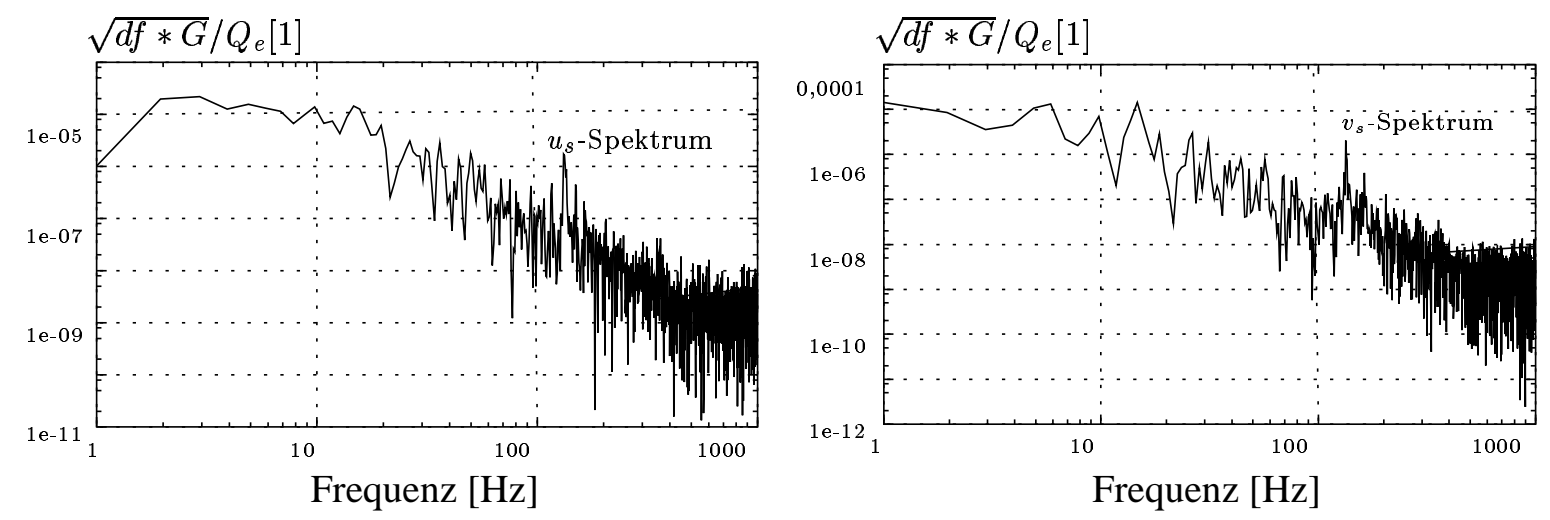

Abbildung 2.2: Leistungspektren des leeren Kanals bei $U_{\infty}=21 \mathrm{~m} / \mathrm{s}$, nach König [38]

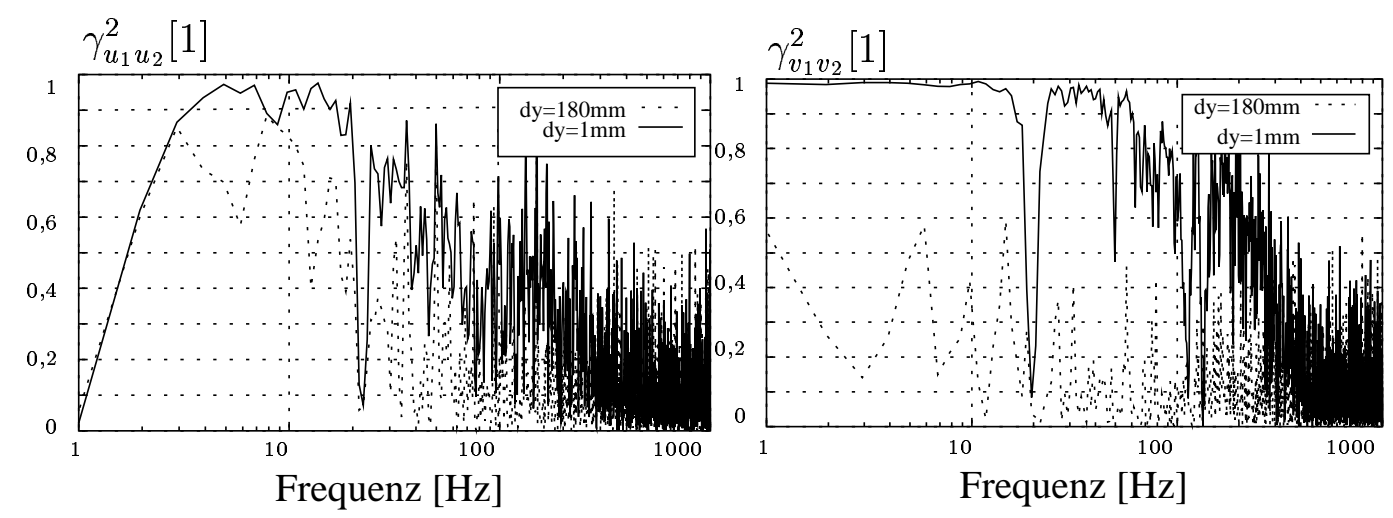

Abbildung 2.3: Kohärenzfunktion des leeren Kanals bei $Q_{e}=21 \mathrm{~m} / \mathrm{s}$ über verschiedene spannweitige Entfernungen dy, nach König [38]

Ursache dafür sind Druckschwankungen, hervorgerufen durch Wechselwirkungen von Kanaleinbauten wie Umlenkbleche und Siebe mit dem Gebläse in Form von Strömungsablösungen an den Schaufeln und die akustische Eigenresonanz des Kanals bei den Frequenzen $f_{n}$

$$
\frac{c}{f_{n}}=n \lambda ; \quad c: \text { Schallgeschwindigkeit }, \lambda=98 m, n=1,2,3, \ldots
$$

Oberhalb dieser Frequenzen dominieren sowohl in Hauptströmungsrichtung als auch in Querströmungsrichtung lediglich kleinskalig kohärente Geschwindigkeitsfluktuationen bis etwa $400 \mathrm{~Hz}$.

Die Tabelle 2.2 faßt die Ergebnisse zusammen. Die Untersuchungen wurden bei einer Geschwindigkeit von $U_{\infty}=21 \mathrm{~m} / \mathrm{s}$, einem Frequenzbereich von 1-2000 Hz und bei geschloßener Meßstrecke durchgeführt. Der korrelierte Anteil wurde über die spannweitige Entfernung von $d y=0,08 m$ bestimmt. Die statistischen Eigenschaften der Schwankungsgrößen in Querrichtung, $v^{\prime}$ und $w^{\prime}$, wurden als identisch angenommen. 


\begin{tabular}{|c|c|c|c|}
\hline norm. Gesamtturbulenz & $u^{\prime}=0,0006$ & $v^{\prime}=0,0010$ & $T u=0,00084$ \\
\hline norm. langskalig korrelierter Anteil & $u_{a}^{\prime}=0,0003$ & $v_{a}^{\prime}=0,0004$ & $T u_{a}=0,0004$ \\
\hline norm. Wirbelstärkeschwankungen & $u_{v}^{\prime}=0,0005$ & $v_{v}^{\prime}=0,0009$ & $T u_{v}=0,0008$ \\
\hline
\end{tabular}

Tabelle 2.2: Integrale Werte der Turbulenz des NWB bei geschlossener Meßstrecke normiert auf $U_{\infty}=21 \mathrm{~m} / \mathrm{s}$

\begin{tabular}{|c|c|}
\hline Spannweite & $\mathrm{L}=1,5 \mathrm{~m}$ \\
\hline Tiefe & $\mathrm{c}=0,65 \mathrm{~m}$ \\
\hline Dickenverhältnis & $14,8 \%$ \\
\hline Schiebewinkel & $\Phi=45^{\circ}$ \\
\hline Anstellwinkel & $\alpha=-3^{\circ}$ bis $-4^{\circ}$ \\
\hline mittl. Rauhtiefenwert & $\begin{array}{r}R_{z}=25 \ldots 30 \mu \mathrm{m} \text { im Nasenbereich } \\
R_{z}=20 \mu \mathrm{m} \text { Oberseite }\end{array}$ \\
\hline
\end{tabular}

Tabelle 2.3: Geometriedaten des untersuchten Modells

\subsubsection{Das untersuchte Modell}

Das für die Untersuchungen ausgewählte Modell ist ein Tragflügel in Hohlprofilbauweise. Es besitzt ein Dickenverhältnis von $14,8 \%$, das bei $x / c=0,342$ erreicht wird. Das Profil (HQ26) weist eine besonders starke Krümmung auf der Oberseite auf, wie man den Abbildungen 2.4 und 2.5 entnehmen kann.

Das Modell wurde mit einem Schiebewinkel von $45^{\circ}$ mittels profilierter Flansche vertikal in die Mitte der Meßstrecke eingebaut, um ein Maximum der Querströmung zu erhalten. Die Einbauflansche ermöglichen ein stufenloses Justieren des Anstellwinkels im Bereich von $\pm 5^{\circ}$. Für die Untersuchungen wurden jedoch nur Anstellwinkel von $\alpha=-3^{\circ}$ bis $-4^{\circ}$ gewählt, weil sich dadurch der Bereich mit negativem Druckgradient $d p / d x<0$, in welchem die Querströmungsinstabilität den Transitionsprozeß bestimmt, von der Nase bis zu einer Profiltiefe von $x / c=0,4$ entlang der besonders stark konvex gekrümmten Oberseite ausdehnte. Ein positiver Nebeneffekt dieses negativen Anstellwinkels war das fast vollständige Fehlen einer Auftriebskomponente, die ein anströmungsgeschwindigkeitsabhängiges Durchbiegen des Modells zu Folge gehabt hätte. Trotzdem mußte man das Modell an zwei Spannweitenpostionen mit der Meßstreckenwand verspannen, um Biegeschwingungen zu unterdrücken (siehe Abbildung 2.4).

Um für alle im Experiment verwendeten Reynoldszahlen eine Strömungsablösung an 


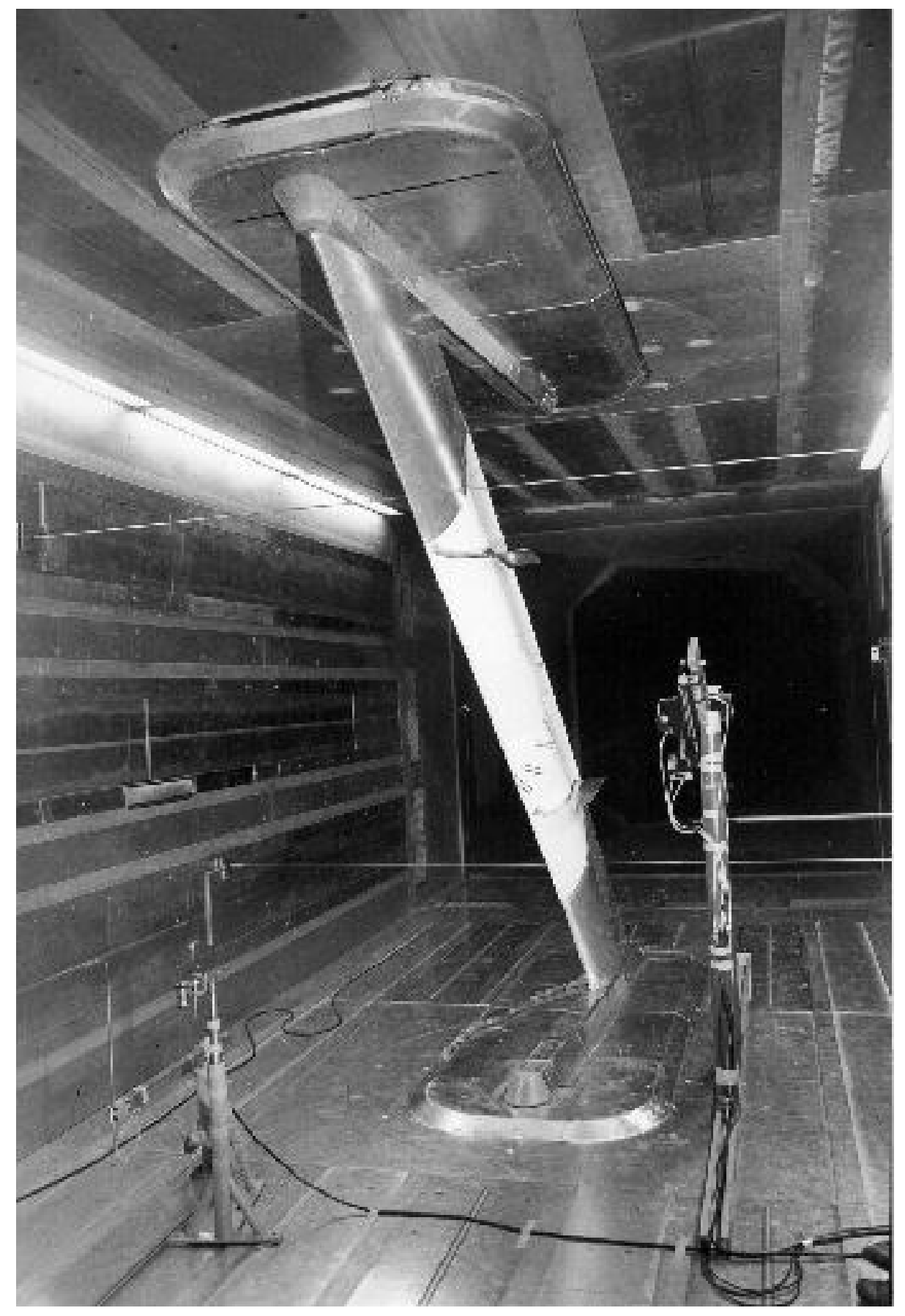

Abbildung 2.4: Foto der Meßstrecke mit eingebautem Modell und Meßstativen 


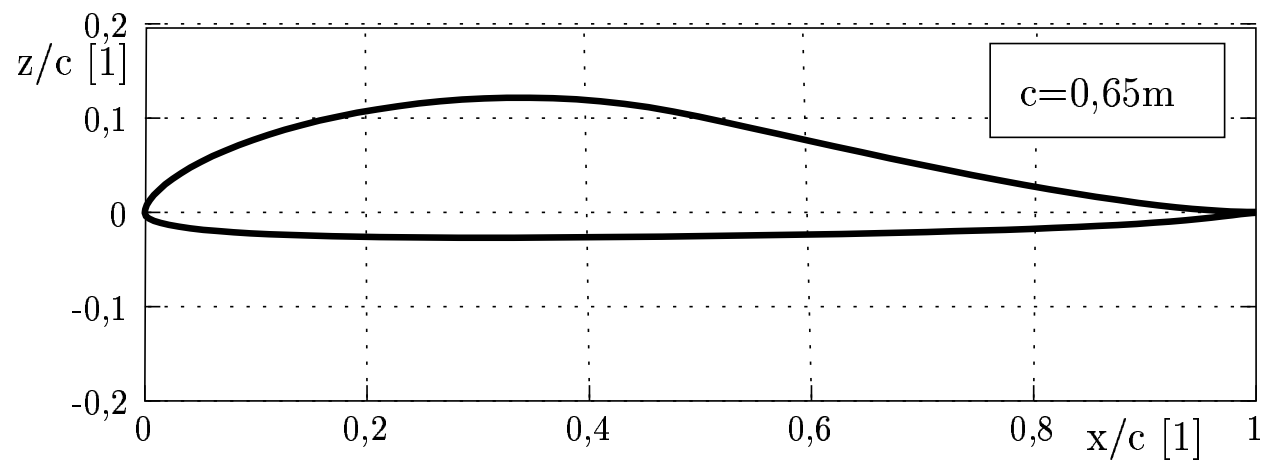

Abbildung 2.5: Querschnitt des Tragflügelprofils HQ26; $d / c=14,8 \%$

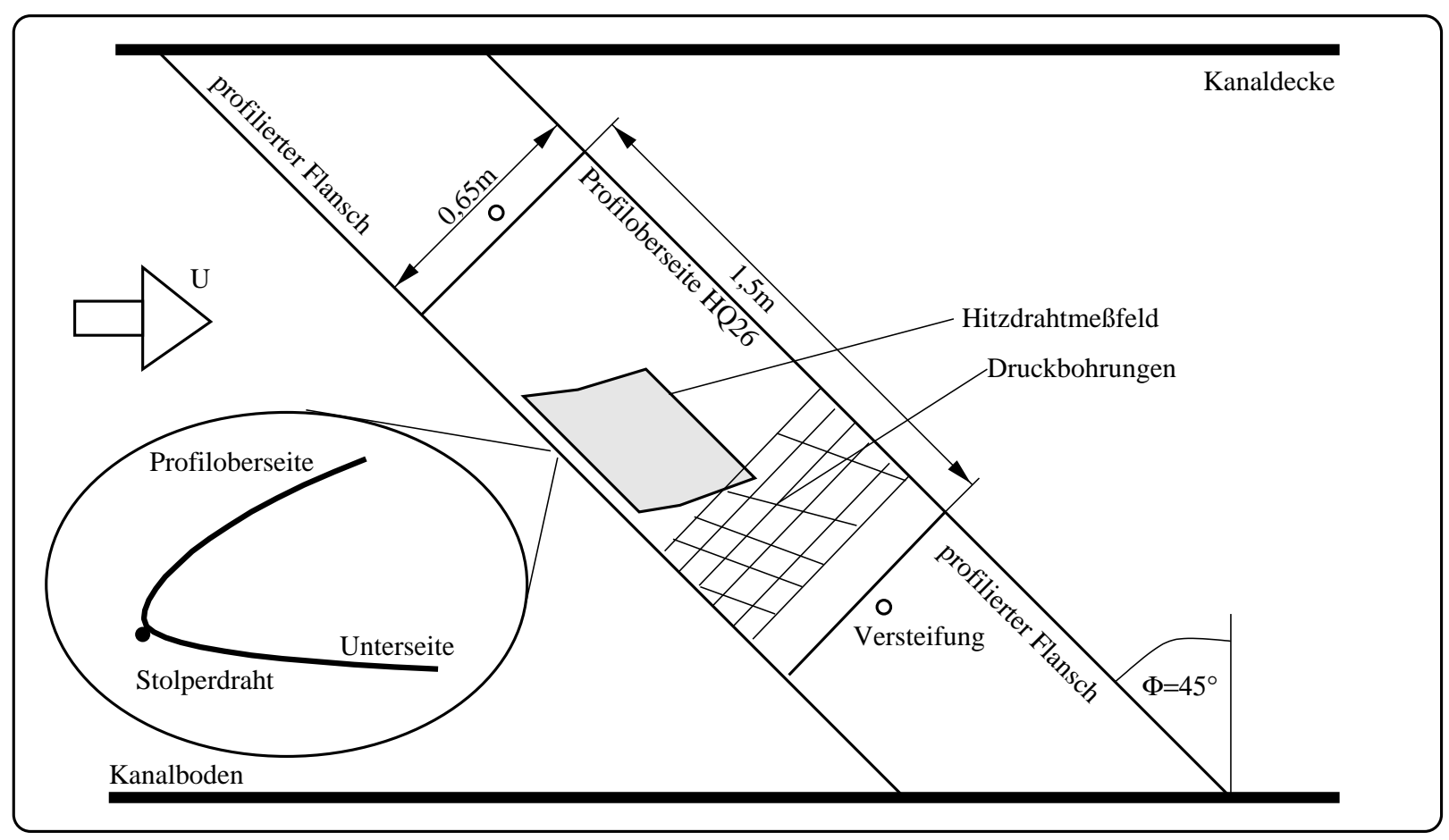

Abbildung 2.6: Schematische Draufsicht auf das in den Kanal montierte Modell 
der Unterseite infolge der durch die starke Krümmung im Vorderkantenbereich entstehende starke Saugspitze zu vermeiden, wurde ein Stolperdraht parallel zur Modellvorderkante angebracht, der die Grenzschicht an der Profilunterseite frühzeitig turbulent werden ließ und so die Ausbildung einer abgelösten Scherschicht bzw. einer lokalen Ablöseblase verhinderte. Diese Ablösungen könnten einerseits die Umströmung instationär beeinflussen und damit Biegeschwingungen des Modells anregen oder die Staulinie oszilieren lassen ${ }^{1}$. Andererseits würde Ablösung die dimensionslose Druckverteilung Reynoldszahl-abhängig machen und damit die Grenzschicht-Ähnlichkeit für verschiedene Anströmungsgeschwindigkeiten zerstören.

Der mittlere Rauhtiefenwert der polierten Modelloberfläche betrug im stark gekrümmten Vorderkantenbereich $R_{z}=25 \ldots 30 \mu \mathrm{m}$ und $R_{z}=20 \mu \mathrm{m}$ in Bereichen schwacher Krümmung. Dieser Wert ist insbesondere für die Anregung stationärer Instabilitätsmoden ein wichtiges Maß. Er ermöglicht den Vergleich mit Ergebnissen anderer Experimente und die Bestimmung der Anfangsamplituden für PSE Rechnungen.

Das Modell ist mit insgesamt 71 Miniaturdruckanbohrungen versehen, davon 41 an der Oberseite und 30 an der Unterseite. Die Druckanbohrungen liegen spannweitig in 6 Ebenen, um Stromabwechselwirkungen der Druckbohrungen zu verhindern (zu erkennen an den Schnittpunkten der Gitterlinien in Abbildung 2.6). Mit ihnen ist es möglich, die Druckverteilung über dem Flügel zu bestimmen und die Grundströmung zu beurteilen.

Am Modell wurden in dem Zeitraum meiner Untersuchungen (November 1995 bis Juni 1997) verschiedene Einbauten im Bereich der Vorderkante vorgenommen. Diese Einbauten änderten die Oberflächenbeschaffenheit im Bereich neutraler Stabilität. Dadurch sind direkte Vergleiche von Meßergebnissen aus den verschiedenen Meßkampagnen beeinträchtigt.

\subsection{Meßtechnik}

Einen schematischen Überblick über die Meßkette gibt Abbildung 2.7. Zur Meßwerterfassung an den Druckanbohrungen auf dem Profil wurde ein Meßstellenumschalter (SCANIVALVE) benutzt, der den Druck der einzelnen Druckbohrungen der Reihe nach auf eine Druckmeßdose SETRA führte. Diese wandelte die Druckdifferenz zum Umgebungsdruck in einen Spannungwert, der über einen A/D-Wandler digitalisiert und auf einem PC abgespeichert wurde. Neben diesem System wurden seitens der Kanalbetreiber auch Spannungsmonitore für die Meßstreckentemperatur, die Kanalgeschwindigkeit anhand des Vorkammerdrucks und eine weitere Druckdose für Eichzwecke zur Verfügung gestellt. Die Meßwerte durch eine Mehrkanal-DAP A/D-Wandlerkarte digitalisiert und als Mittelwerte zusammen mit den zugehörigen Hitzdrahtmessungen aufgenommen.

Zur Detektion des Umschlages in Abhängigkeit von Anströmgeschwindigkeit und Anstellwinkel wurden Infrarotaufnahmen des angeströmten Profil gemacht. Bei dieser Meßtechnik nutzt man die stärkere Erwärmung oder auch den stärkeren Wärmetransport von

\footnotetext{
${ }^{1}$ Beides würde die Grundströmung instationär deformieren bzw. die Instabilitätsbedingungen verändern.
} 


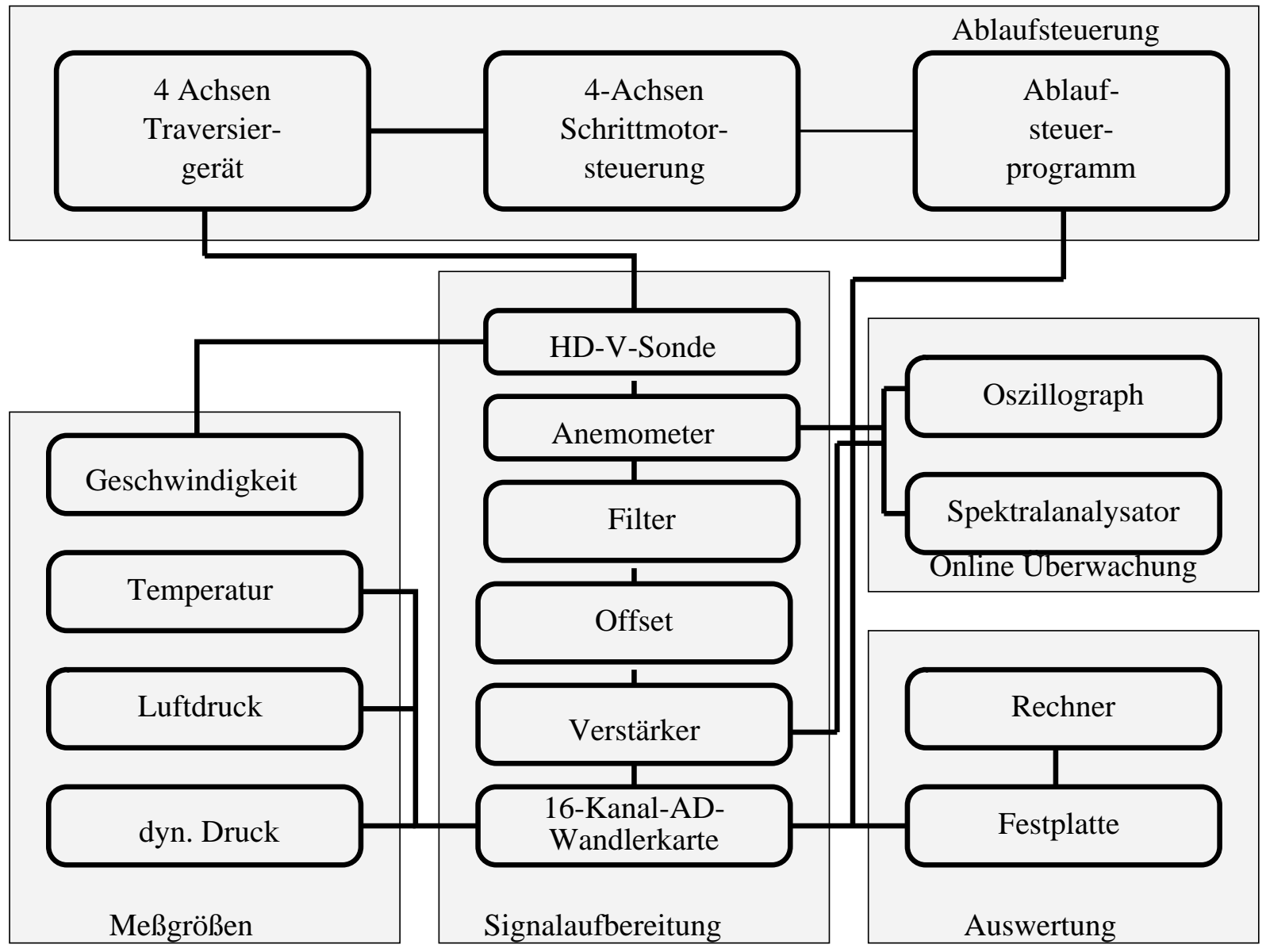

Abbildung 2.7: Blockdiagramm der Meßwertaufnahme im Experiment

turbulenten im Vergleich zu laminaren Wandgrenzschichten aus. Heizt man beispielsweise das Modell gegenueber dem Kanalmedium auf, so wird es an den turbulent überströmten Stellen schneller auskühlen als an den laminar überströmten, und das Bild dieser Stellen wird, bis die Temperaturunterschiede abgeklungen sind, entsprechend dunkler. Die Temperaturdifferenzauflösung der verwendeten Kamera lag bei etwa 0,1K, womit sich dieses Verfahren hauptsächlich für große Geschwindigkeiten eignet. Mit entsprechend optimiertem Signal-Rausch-Verhälnis war aber auch in dem von mir untersuchten Geschwindigkeitsbereich eine Aussage zur Transitionslage möglich.

Zur Messung instationärer Geschwindigkeiten im sub - und supersonischen Bereich verwendet man häufig die Hitzdrahtmeßtechnik ${ }^{2}$. Dabei wird anhand der Wärmeabführung an einem elektrisch überhitzten dünnen Draht auf den Massenfluß des umgebenden strömenden Fluids geschlossen.

\footnotetext{
${ }^{2}$ Tiefergehende Details zu dieser Meßtechnik sowie zu zahlreichen anderen in der Strömungsphysik eingesetzten Meßverfahren findet man in Eckelmann (1997) [39].
} 
Analog zum Kingschen Gesetz

$$
\dot{Q}=\left[A+B\left(\rho u_{\perp}\right)^{1 / n}\right] \Delta T
$$

mit

$\dot{Q}: \quad$ abgeführte Wärmemenge pro Zeiteinheit

$A, B, n$ : durch Kalibrierung zu bestimmende Konstanten

$\Delta T: \quad$ Temperaturdifferenz zwischen Hitzdraht und Umgebungsmedium

$u_{\perp}$ : Geschwindigkeitskomponente normal zur Hitzdrahtoberfläche,

gelingt es, in inkompressiblen Strömungen ( $\rho=$ const.) aus der dem Hitzdraht zugeführten Wärme und bei Kenntnis der Sonden- und Fluidtemperatur auf die Anströmungsgeschwindigkeitskomponente normal zur Hitzdrahtoberfläche zu schließen. Kombiniert man zwei gemeinsam eine Ebene aufspannende Hitzdrähte, so kann man die Projektion des Anströmungsgeschwindigkeitsvektors in diese Ebene messen, unter der Voraussetzung eines räumlich über die geometrische Abmessungen der beiden Hitzdrähte quasikonstanten Geschwindigkeitsvektors.

Zur Ermittlung der abgeführten Wärme hält man mittels schneller Widerstandsmeßbrücken die Temperaturdifferenz $\Delta T=$ const. und mißt die dazu nötige Heizspannung (Konstanttemperaturanemometrie). Dazu wurden die Meßbrücken 55M10 der Firma DISA verwendet. Sie gestatten (lt. Handbuch) bei sorgfältigem Abgleich, die Frequenzen bis $f=100 k H z$ aufzulösen. Für die Strömungssituation im vorliegenden Experiment genügte allerdings schon eine Grenzfrequenz von $f=12 k H z$, so daß die Grenzauflösung nicht überprüft werden mußte.

Die Ausgangsspannungen dieser Meßbrücken wurden verstärkt und mit einem Offset versehen, um den festen Eingangsspannungsbereich der Digitalisiereinheit $( \pm 5 \mathrm{~V})$ bestmöglich auszusteuern. Als A/D-Wandler wurde eine 16 Kanal - 12 bit - Wandlerkarte (4096 Quantisierungsstufen) verwendet. Diese Karte verfügt über einen Onboard - Speicher von 64kB pro Kanal und gestattete somit, je nach gewählter Abtastfrequenz, Meßzeiten von $T \geq 2 s$. Die verwendete zeitliche Auflösung wurde den Erfordernissen aus dem Abtasttheorem angepaßt. Sie lagen im Experiment zwischen $4000 \mathrm{~Hz}$ und $12000 \mathrm{~Hz}$.

Ein Tiefpaßfiltern vor der Digitalisierung war für die Mehrzahl der Untersuchungen nicht nötig, da die Signalkomponenten oberhalb der halben gewählten Samplingfrequenz auf Grund ihrer kleinen Amplitude nicht mehr durch den A/D-Wandler aufgelöst wurden. Lediglich zur Untersuchung des Zusammenbruchs der laminaren Strömung wurden Filter verwendet. Das Abschätzen der Amplituden der Signalanteile oberhalb der halben Abtastfrequenz erfolgte mit Hilfe eines digitalen Spektralanalysators der Firma Advantest, Modell $\mathrm{R} 9211 \mathrm{~A} / \mathrm{E}$.

Bei den verwendeten Hitzdrahtsonden handelte es sich um spezielle V-Miniatursonden (siehe Abbildung 2.8). Mit ihnen kann man die Projektion des momentanen Anströmungsvektors in die durch die Hitzdrähte aufgespannte Ebene in einem Winkelbereich von $\pm 45^{\circ}$ bezüglich der Sondensymmetrieachse bestimmen. Der verwendete Hitzdrahtdurchmesser 

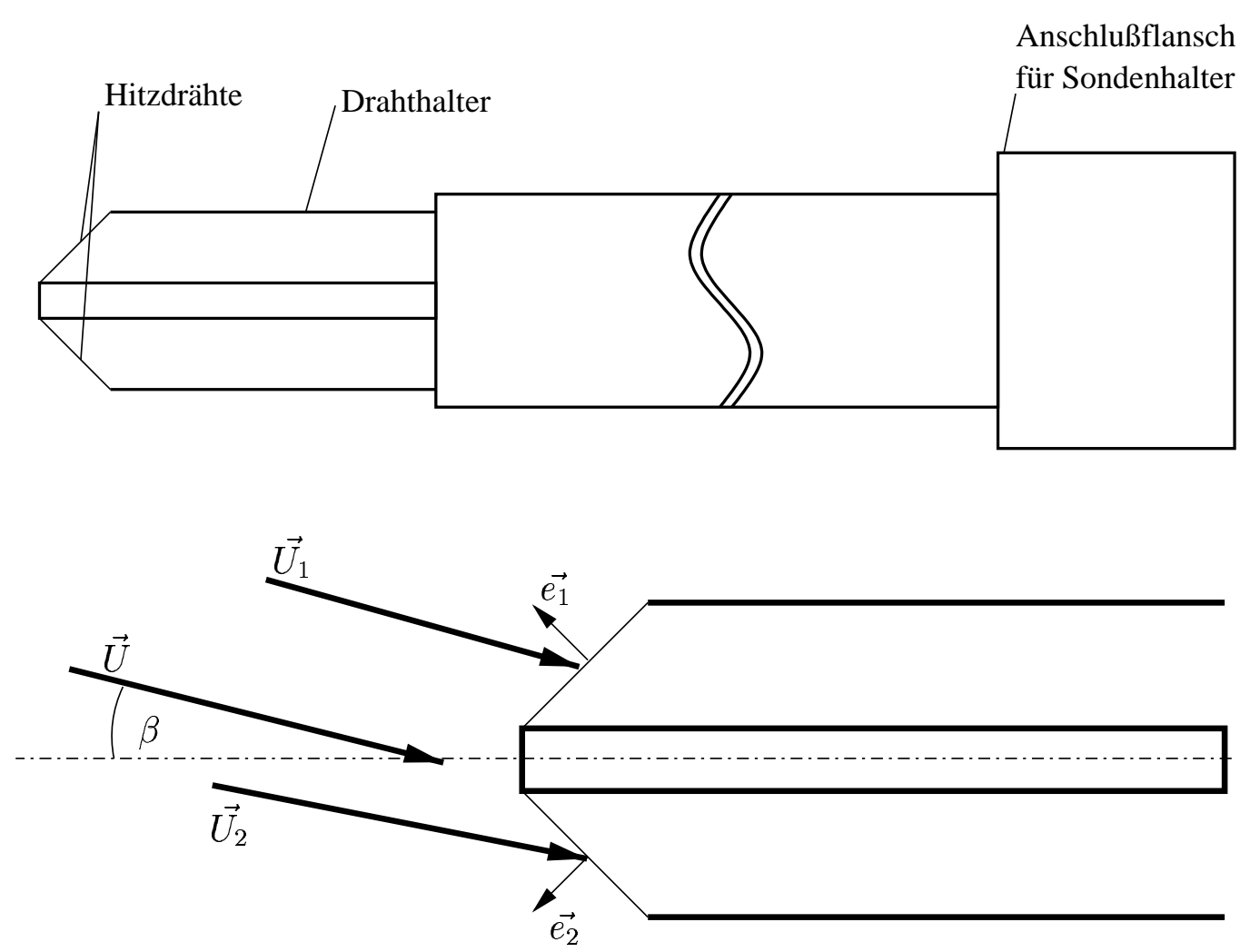

Abbildung 2.8: Prinzipskizze einer Hitzdraht-V-Sonde

betrug $5 \mu m$, und der Abstand Drahtmitte-Drahtmitte der beiden Hitzdrähte lag bei ca. einem Millimeter. Die kleinen Abmessungen waren nötig, um die erwarteten räumlich kleinen Strukturen auflösen zu können.

\subsubsection{Hitzdrahtmessungen mit V-Sonden in stationären und in- stationären Geschwindigkeitsfeldern}

Das Meßsignal einer Zwei-Draht-Hitzdraht-Grenzschichtsonde besteht aus zwei Spannungen. Aus ihnen kann man in Verbindung mit einer Geschwindigkeits- und Winkelkalibrierung und unter Annahme lokaler Parallelströmung die zwei Komponenten der lokalen Geschwindigkeit in der durch die zwei Einzelhitzdrähte aufgespanneten Ebene messen ${ }^{3}$. Wählt man die Ebene z.B. parallel zur Modeloberfläche, dann lassen sich aus solchen Messungen die Geschwindigkeitskomponenten $u$ und $v$ berechnen, also die beiden Komponenten, aus denen sich das verwundene Querströmungsprofil zusammensetzt. Dabei setzt man eine quasikonstante Geschwindigkeit über der Sondengeometrie voraus. Diese Näherung gilt

\footnotetext{
${ }^{3}$ Rosemann gibt in [40] eine allgemeine Einführung zum Problem der Messungen mit Mehrfachhitzdrahtsonden
} 
mit Sicherheit in der freien Strömung. In der Grenzschicht mit ihren starken wandnormalen Geschwindigkeitsgradienten gilt sie, wenn überhaupt, nur für Ebenen parallel zur Oberfläche. Das erfordert große Sorgfalt beim wandparallelen Justieren der Hitzdrahtsonde.

Besitzt die Grenzschicht zusätzlich zum wandnormalen Geschwidigkeitsgradienten noch einen signifikanten spannweitigen Geschwindigkeitsgradient, so kann man die Näherung einer über die Sonde räumlich quasikonstanten Geschwindigkeit nur noch für die zeitlichen Mittelwerte aufrechterhalten, indem man zwei Messungen durchführt, bei denen man die Sonde so positioniert, daß die einzelnen Hitzdrähte nacheinander an den selben Ort im Geschwindigkeitsfeld gebracht werden (Zweipunktmessung).

In den Auswertungen zum vorliegenden Experiment geht es häufig um instationäre Störungen mit hoher räumlicher Kohärenz, wie z.B. laufende Instabilitätswellen. Um diese von den ebenso vorhandenen, nur kurzskalig kohärenten Geschwindigkeitsfluktuationen zu trennen, wie sie als Folge turbulenter Spots in den Meßsignalen enthalten sein können, habe ich ein Verfahren zur Berechnung von Frequenzspektren benutzt, bei dem zunächst für beide Einzelhitzdrähte eine Geschwindigkeitskalibrierung durchgeführt wird. Bildet man von den resultierenden effektiven Kühlgeschwindigkeiten der Einzelhitzdrähte ein Kreuzspektrum (incl. Hammingfensterung mit Overlap-Add), so erhält man in den spektralen Amplituden ein Maß für die im Signal enthaltenen langskalig kohärenten Geschwindigkeitsfluktuationen. Da diese Amplituden im Wesentlichen durch Instabilitätswellen hervorgerufen werden, hat man damit eine Größe extrahiert, die sich gut zum Vergleich mit den Ergebnissen von Instabilitätsanalysen eignet, in denen per Definition nur die Entwicklung großskalig vorhandener Störungen (eben Instabilitätswellen) betrachtet wird.

\subsubsection{Bestimmung der Wellenzahlvektoren instationärer Instabi- litätswellen}

Im Falle instationärer Messungen können sich zwei Probleme ergeben. Erstens muß auch hier sichergestellt werden, daß die Sonde klein gegen die Struktur der zu messenden Störung ist. Im Falle einer instationären Störung in Form einer ebenen Welle und einer spannweitigen Ausdehnung der Sonde von $1 \mathrm{~mm}$ bedeutet das:

$$
\lambda_{\|} / 4<<1 m m \text {. }
$$

( $\lambda_{\|}$ist die auf die spannweitige Richtung der Sonde projezierte Wellenlänge.) Zweitens kann kann es vorkommen, daß die Amplitude der instationären Störung mit der Amplitude der stationären Störung moduliert ist. In einem solchen Fall muß man sicherstellen, daß auch für die stationäre Wellenlänge die Bedingung 2.4 gilt.

Kann man diese Bedingung nicht ausreichend erfüllen, bietet sich zur Bestimmung der Charakteristika der instationären Strömungsanteile ein anderes Verfahren an.

Ausgehend von der Annahme, daß die beiden einzelnen Hitzdrähte einer Grenzschicht-VSonde sich nicht beeinflussen, kann man sie als zwei dicht nebeneinander stehende Eindrahtsonden auffassen. Für die gegebenen Normalenvektoren $\vec{e}$ der einzelnen Hitzdrähte 


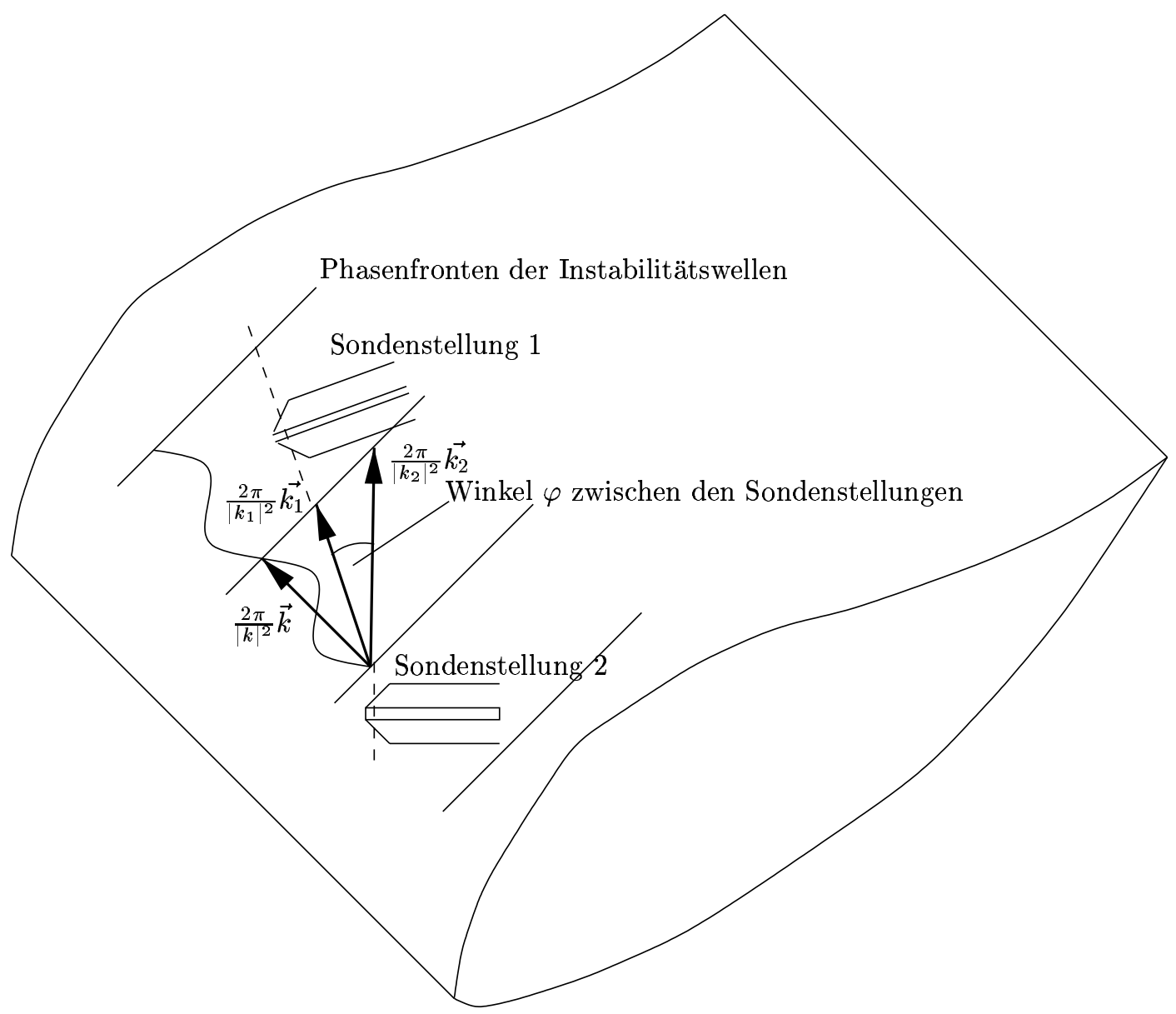

Abbildung 2.9: Meßprinzipskizze zur Bestimmung der Wellenzahlvektoren von Instabilitätswellen 
mißt man in erster Näherung die effektiven Kühlgeschwindigkeiten

$$
\begin{aligned}
& U_{\text {eff } 1}=\overrightarrow{e_{1}} \cdot \overrightarrow{U_{1}} \\
& U_{e f f 2}=\overrightarrow{e_{2}} \cdot \overrightarrow{U_{2}}
\end{aligned}
$$

Damit kann man aus den experimentell bestimmten Phasendifferenzen zwischen $U_{\text {eff } 1}$ und $U_{e f f 2}$ unter Annahme zeitlicher harmonischer Störungen in Form ebener Wellen auf die Phasendifferenzen der die beiden Hitzdrähte kühlenden lokalen Anströmungsvektoren $\vec{U}_{1}$ und $\vec{U}_{2}$ schließen.

Für die Hitzdraht-V-Sonden mit Drahtschiebewinkeln von $\beta= \pm 45^{\circ}$ gilt für den Zusammenhang der Phasendifferenzen von $U_{\text {eff }}$ und $\vec{U}_{n}$

$$
\varphi\left(U_{e f f 1}, U_{e f f 2}\right)=-\pi / 2+\varphi\left(\vec{U}_{1}, \vec{U}_{2}\right)
$$

für

$$
\begin{array}{r}
\vec{U}=\left(\bar{U}+u \cdot e^{i \omega t}\right) \overrightarrow{e_{x_{s}}}+v \cdot e^{i \omega t} \vec{e}_{y_{s}} \\
v=u \cdot e^{-i \pi / 2}
\end{array}
$$

Gilt $v \ll u$ so erhält man als Zusammenhang

$$
\varphi\left(U_{e f f 1}, U_{e f f 2}\right)=\varphi\left(\vec{U}_{1}, \vec{U}_{2}\right)
$$

und für $u \ll v$

$$
\varphi\left(U_{e f f 1}, U_{e f f 2}\right)=\pi+\varphi\left(\vec{U}_{1}, \vec{U}_{2}\right) .
$$

Dreht man die Hitzdrahtsonde zur Anströmung, so ändert sich die Phasenverschiebung zwischen den Signalen der Einzelhitzdrähte. Aus der Differenz der beiden gemessenen Phasendifferenzen kann man die Ausrichtung der Phasenfronten der instationären Störungen sowie deren Wellenlänge ermitteln (siehe Abbildung 2.9) ${ }^{4}$. Die Richtung der Vektoren $\overrightarrow{k_{1}}$ und $\overrightarrow{k_{2}}$ ist durch die Stellung der Sonden vorgegeben, die Länge berechnet sich aus

$$
\lambda=\frac{2 \pi}{\left|k_{n}\right|}=\Delta s \frac{2 \pi}{\Delta \varphi}
$$

$\Delta \varphi$ : gemessene Phasendifferenz zwischen den beiden Hitzdrähten einer V-Sonde. $\Delta s$ : Abstand Drahtmitte $\leftrightarrow$ Drahtmitte der beiden Hitzdrähte einer V-Sonde. Damit ergibt sich für die Wellenlänge der Instabilitätswellen

$$
\lambda=\frac{2 \pi}{\left|k_{1}^{2}\right|} \overrightarrow{k_{1}} \cdot \frac{2 \pi}{\left|k_{2}^{2}\right|} \overrightarrow{k_{2}}
$$

${ }^{4}$ Dabei hat man allerdings sicherzustellen, daß für jede Sondenausrichtung eine bekannte Anzahl von projizierten Wellenlängen zwischen den beiden Einzelhitzdrähten liegt. Darüber verschafft man sich Klarheit, indem man die Sonde zunächst in kleinen Winkeln zur Anströmung dreht und die ermittelte Phasendifferenz über den Sondenwinkel aufträgt: Aus dem Anstieg läßt sich nun die Anzahl dazwischenliegender Maxima/Minima ermitteln. 
und für den Winkel des Wellenvektors bzgl. Sondenposition 2:

$$
\varphi_{\left(\overrightarrow{k_{2}}, \vec{k}\right)}=\arcsin \left(\frac{\left|\overrightarrow{k_{2}}\right|}{|\vec{k}|}\right)
$$

Zur Detektion der Phasendifferenz verwendet man das Kreuzspektrum der geschwindigkeitskalibrierten Hitzdrahtspannungen. Für Fensterfunktion und Vorschub kann man Standardverfahren verwenden, da keine große Dynamik im Frequenzverlauf vorliegt (hier wurden Hamming Fenster verwendet und das Fenster jeweils ein Drittel weitergeschoben). Eine ungenügende Anzahl von Mittelungen erkennt man an relativ großen Streuungen der Phasendifferenzen über der Frequenz. Durch die Mittelung der Einzelspektren werden die in Grenzschichten vorhandenen statistischen Signalanteile unterdrückt, und es akkumulieren sich systematisch auftretende, zwischen beiden Hitzdrähten kohärente Ereignisse. Das Maß der Kohährenz kann man sich anhand der Kohärenzfunktion

$$
\gamma^{2}:=\frac{\left(S_{A}^{*} \cdot S_{B}\right)\left(S_{B}^{*} \cdot S_{A}\right)}{\left(S_{A}^{*} \cdot S_{A}\right)\left(S_{B}^{*} \cdot S_{B}\right)}
$$

mit

$$
S_{X}(f)=\mathcal{F}\left\{s_{X}(t)\right\}
$$

ansehen. $s_{X}(t)$ sei das Geschwindigkeitssignal von Hitzdraht $X$. Sie gibt den Bruchteil korrelierter Leistung im Signal an.

Die Grundlage der hier geschilderten Auswerteverfahren von Messungen mit Grenzschichthitzdrahtsonden ist die anströmungsrichtungsabhängige Kühlung der Sonden über das Skalarprodukt von Hitzdrahtnormalen und Anströmung. Dieses gilt allerdings nicht mehr für sehr große Anströmungswinkel. Man muß also einen Kompromiß eingehen, z.B. zwischen der Wahl großer Sondenwinkeldifferenzen und kleiner Hitzdrahtschiebewinkel. In den hier durchgeführten Messungen wurden Sondenwinkel von $\beta \approx \pm 12^{\circ}$ gewählt. 


\section{Kapitel 3}

\section{Grundströmungsentwicklung}

\subsection{Notationen}

\subsubsection{Die verwendeten Koordinatensysteme}

Die beiden wichtigen Koordinatensysteme sind in Abbildung 3.1 dargestellt.

Das modellfeste Koordinatensystem wird mit dem Index c bezeichnet. $y_{c}$ zeigt parallel zur Vorderkante, $x_{c}$ entlang der Profilsehne von der Vorderkante nach hinten. $z_{c}$ zeigt aus der

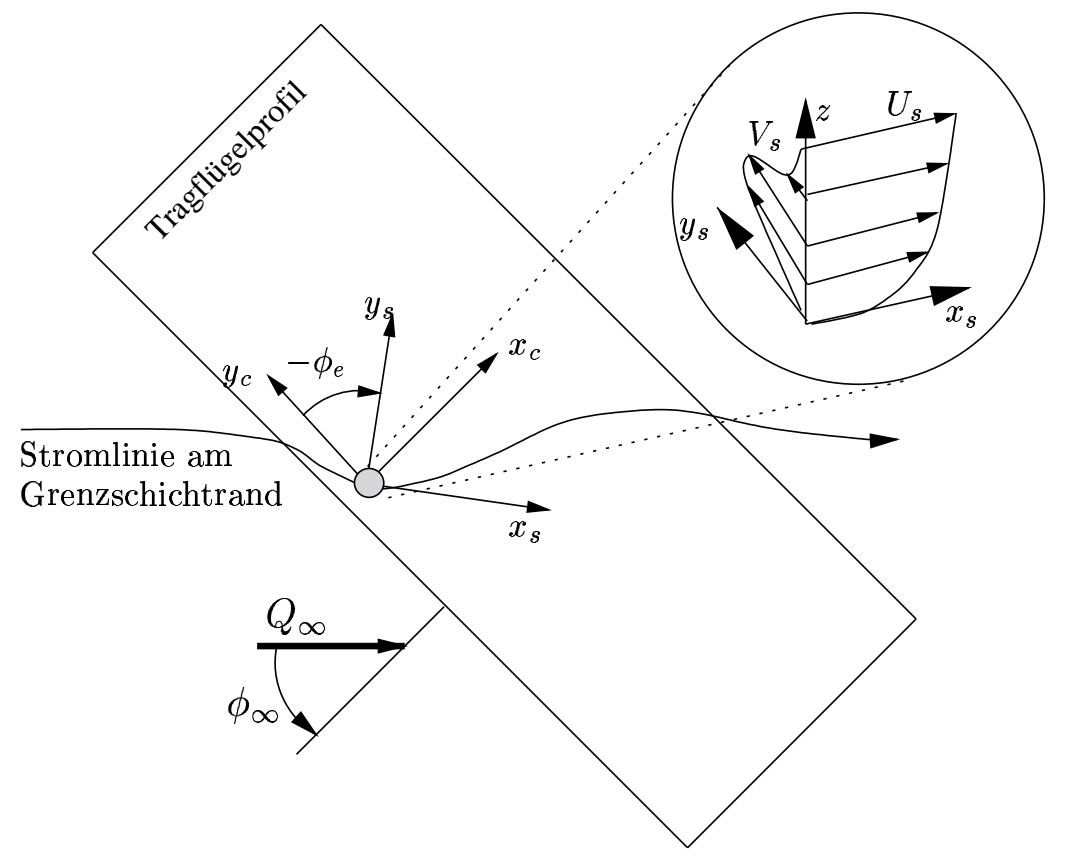

Abbildung 3.1: Skizze der verwendeten Koordinatensysteme 
Modellebene senkrecht nach oben und gibt somit den Wandabstand an. Streng genommen ist es damit kein orthogonales Koordinatensystem, da $z$ nicht exakt rechtwinklig auf der $x_{c}-y_{c}$-Ebene steht. Praktisch macht sich dieser Unterschied allerdings nur in unmittelbarer Nähe der Vorderkante bemerkbar und ist deshalb hier vernachlässigbar.

Das Stromlinienkoordinatensystem ist mit dem Index s bezeichnet. $x_{s}$ zeigt entlang der Stromline am Grenzschichtrand, $y_{s}$ verläuft orthogonal zu $x_{s}$ und parallel zur Oberfläche. $z_{s}$ zeigt analog zu $z_{c}$ normal aus der Modelloberfläche heraus. Der Vorteil dieses Koordinatensystems besteht darin, daß außerhalb der Grenzschicht die Querkomponente der Geschwindigkeit verschwindet. Man beachte, daß der Winkel $\varphi$, der die Koordinatensysteme ineinander transformiert, eine Funktion von $x_{c}$ ist. Desweiteren wird der Index von $\mathrm{z}$ meist weggelassen. Fehlt jeglicher Index, so ist das modellfeste Koordinatensystem gemeint.

\section{Die verwendeten Symbole}

$$
\begin{array}{ll}
\vec{U}(x, y, z, t) & \text { Geschwindigkeitsvektor } \\
& \text { am Ort }(\mathrm{x}, \mathrm{y}, \mathrm{z}) \text { zur Zeit t } \\
\overrightarrow{\vec{U}}(x, y, z)=(U, V, W)(x, y, z) & \text { zeitlich gemittelte Geschwindigkeitskomponenten } \\
& \text { am Ort }(\mathrm{x}, \mathrm{y}, \mathrm{z}) \\
(u, v, w)(x, y, z, t) & \text { Schwankungsgrößen der lokalen Geschwindigkeit } \\
& \text { am Ort (x,y,z) zur Zeit t, } \\
& (u, v, w)(x, y, z)=\vec{U}-(U, V, W) \\
& \text { in y-Richtung gemittelte Geschwindigkeit (entspricht } \\
& \text { im Rahmen der linearen Näherung der ungestörten } \\
& \text { laminaren Grenzschichtströmung) } \\
& \text { Anströmgeschwindigkeit } \\
U_{\infty} & \text { Schiebewinkel des Tragflügelprofils } \\
\phi_{\infty} &
\end{array}
$$

Der Index e bezeichnet den Wert der entsprechenden Größe am Grenzschichtrand.

\section{$3.2 \quad$ Grundströmung}

Unter der Grundströmung versteht man die sich ausbildende zeitlich gemittelte Grenzschichtströmung und die sich an sie anschließende Potentialströmung unter Vernachlässigung der Auswirkungen sich entwickelnder Instabilitäten. Die genaue Rekonstruktion der vorhandenen Grundströmung im Experiment ist von entscheidender Bedeutung für den 
Vergleich von Ergebnissen numerischer Stabilitätsanalysen mit experimentellen Resultaten, da die sich entwickelnden Instabilitäten der Grundströmung überlagert sind. Deren Größe läßt sich dabei nur als Differenz aus Gesamtströmung und Grundströmung bestimmen.

Für numerische Stabilitätsanalysen wird in einem ersten Schritt zunächst aus den Druckverteilungen um ein Profil die Potentialströmung rekonstruiert. Aus ihr wird dann in einem zweiten Schritt die Grenzschicht berechnet und für diese schließlich eine (primäre) Stabilitätsanalyse durchgeführt.

Im Falle der Strömung um einen unendlich schiebenden Tragflügel handelt es sich um eine quasi zweidimensionale Grundströmung

$$
\overline{\vec{U}}\left(x_{c}, y_{c}, z_{c}\right)=\overline{\vec{U}}\left(x_{c}, z_{c}\right)
$$

da in Folge des Fehlens jeglicher spannweitiger Gradienten für vorgegebenes $x_{c}$ und beliebiges $y_{c}$ identische zeitlich gemittelte Grenzschichtprofile vorliegen.

\subsection{Potentialströmung}

Die Potentialströmung um das Profil stellt, zusammen mit der Haftbedingung auf der Modelloberfläche, gewissermaßen die Randbedingung für die Grenzschicht dar. Sie prägt der Strömung eine Druckverteilung auf, die in erster Näherung in der gesamten Grenzschicht wirkt und die an der Tragflügelwand gemessen werden kann.

Wird diese Druckverteilung mit dem Staudruck normiert, erhält man bei anliegender Strömung einen nahezu anströmungsgeschwindigkeitsunabhängigen Verlauf. Dieser normierte Druck wird in der Literatur als Druckbeiwert $c_{p}$ bezeichnet.

$$
c_{p}=\left(p_{w}-p_{\infty}\right) /\left(\frac{\rho}{2} U_{\infty}^{2}\right)
$$

Mit Kenntnis der Druckbeiwertverteilung ist es im Rahmen der Prandtlschen Grenzschichtannahmen möglich, die Potentialströmung am Grenzschichtrand zu rekonstruieren. Unter der Annahme einer quasi zweidimensionalen Potentialströmung und der Wahl des körperorientierten Koordinatensystems $\left(x_{c}, y_{c}, z_{c}\right)$, dessen x-Koordinate in Profiltiefenrichtung, die y-Koordinate parallel zur Vorderkante und die z-Koordinate normal zur Modelloberfläche in Richtung Außenströmung ausgerichtet ist, gilt:

- Vorderkantenparallel existieren keine Gradienten

$$
V_{c, e}=U_{\infty} \sin \left(\phi_{\infty}\right)=\text { const. }
$$

- In x-Richtung gilt nach Bernoulli

$$
U_{c, e}=U_{\infty} \cos \left(\phi_{\infty}\right) \sqrt{1-c_{p}}
$$




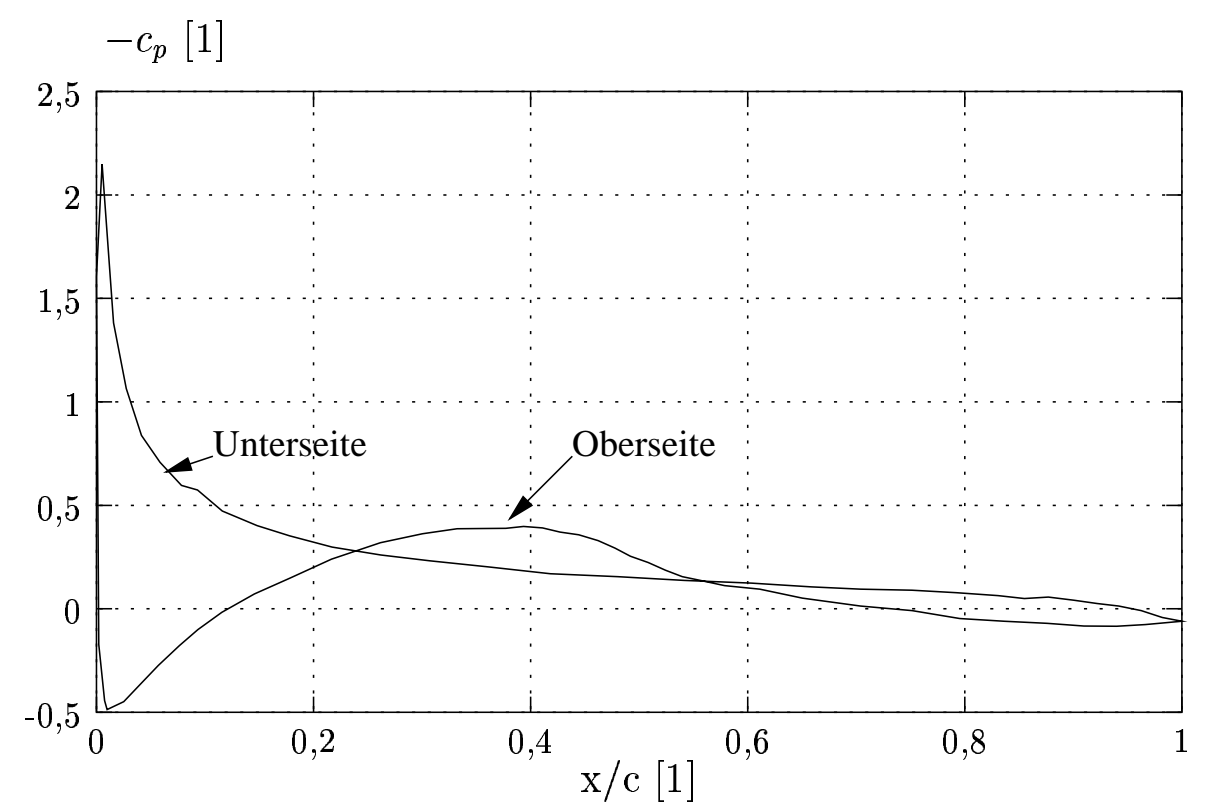

Abbildung 3.2: Druckverteilung um das untersuchte Tragflügelprofil HQ26, $U_{\infty}=28 \mathrm{~m} / \mathrm{s}$, $\alpha=-3.5^{\circ}$

Für den lokalen Winkel der Stromlinie am Grenzschichtrand ergibt sich somit

$$
\phi_{e}\left(x_{c}\right)=\arctan \left(\frac{\tan \phi_{\infty}}{\sqrt{1-c_{p}\left(x_{c}\right)}}\right) .
$$

Die Änderung der Strömungsrichtung mit $c_{p}\left(x_{c}\right)$ führt zu gekrümmten Stromlinien (siehe Abbildung 3.1 auf Seite 23).

Eine exemplarische Druckverteilung über dem untersuchten Tragflügelprofil ist in Abbildung 3.2 dargestellt. Man erkennt in ihr den über einen weiten $x_{c} / c$-Bereich $(0,01 \leq$ $\left.x_{c} / c \leq 0,35\right)$ negativen Druckgradienten der Strömung entlang der Profiloberseite. Es ist somit gelungen, eine Profilumströmung zu realisieren, die über einen weiten Bereich beschleunigt ist und eine Querströmungskomponente aufweist. Wie in Kapitel 4 gezeigt wird, ist unter diesen Bedingungen die Querströmungsinstabilität die dominierende Instabilität.

Die aus Abbildung 3.2 berechneten lokalen Strömungswinkel am Grenzschichtrand sind in Abbildung 3.3 dargestellt. Auf einen Vergleich der experimentell bestimmten Strömungswinkel am Grenzschichtrand und aus den Druckverteilung berechneten Winkeln in Abbildung 3.3 verzichte ich an dieser Stelle. Die experimemtell ermittelten Werte weisen einen hohen zufälligen Fehler auf, da es am Modell keine ausreichend genaue Möglichkeit gab, den Sondenwinkel gegen die Kanalachse zu justieren. Diese Ungenauigkeit hat im weiteren aber keine Auswirkung, da alle Darstellungen stets im Stromlinienkoordinatensystem erfolgen. Der Vergleich der ermittelten Grenzschichtrandgeschwindigkeiten ist der Abbildung 3.6 auf Seite $31 \mathrm{zu}$ entnehmen. Es zeigt sich, daß eine zufriedenstellende Übereinstim- 


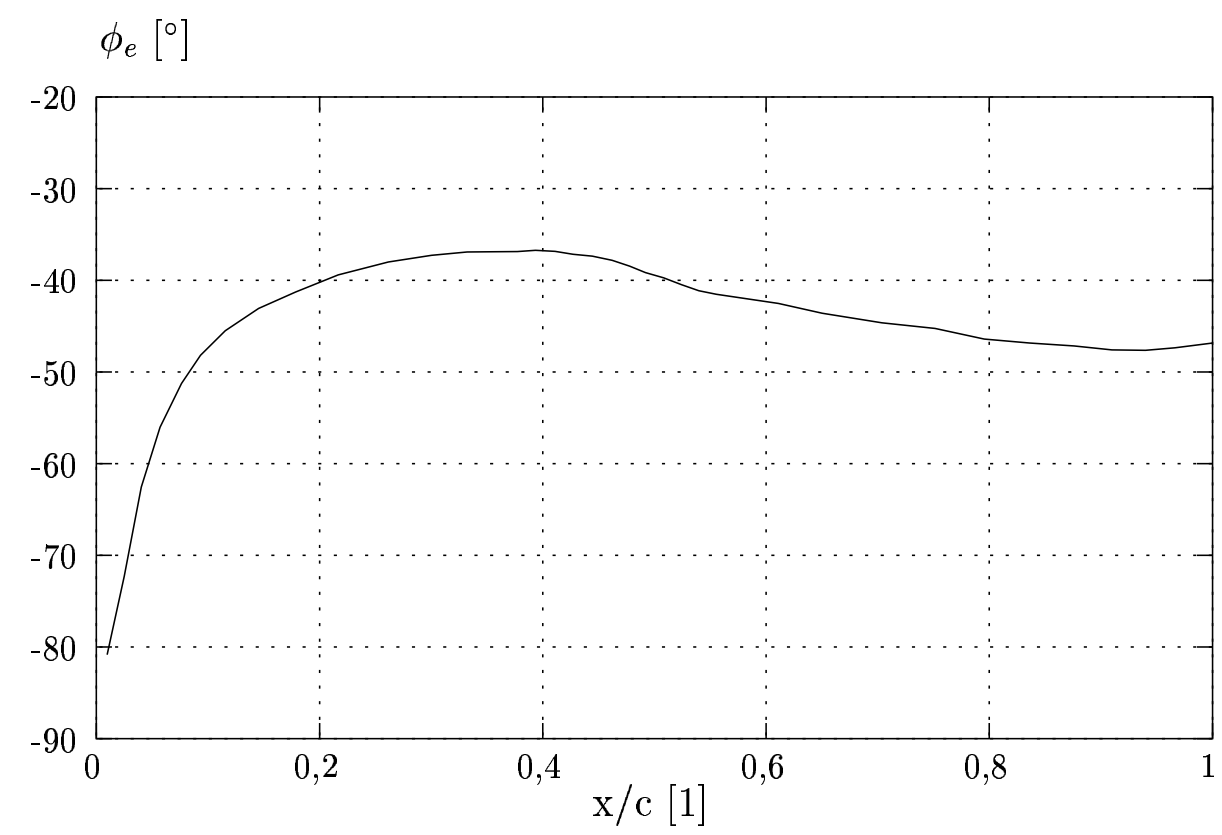

Abbildung 3.3: Lokaler Antrömwinkel am Grenzschichtrand der Profiloberseite

mung erreicht wurde. Daß Druckverteilung und Hitzdrahtmeßwerte an unterschiedlichen spannweitigen Positionen aufgenommen wurden und trotzdem eine gute Übereinstimmung ergaben, läßt auf eine über einen langen spannweitigen Bereich zweidimensionale Potentialströmung schließen und realisiert damit in guter Näherung einen unendlich schiebenden Flügel. Leider wurde diese Strömung durch den Einbau der Sondentraversiereinrichtung in einem Abstand von einem Meter von der Modelloberseite gestört. Insbesondere das Verschiebegrät hat durch seine große Versperrung eine Änderung der Potentialströmung um das Modell zur Folge. Diese Änderung besitzt ihr Maximum auf der potentialströmungsnormalen Projektionsfläche der Traversiereinrichtung auf dem Modell, was man durch Vorbeifahren des Verschiebegeräts an den Modelloberflächendruckbohrungen leicht verifizieren konnte. Glücklicherweise liegt der Ort der Hitzdrahtmessungen sowohl oberhalb als auch vor der Projektionsfläche der Traversiereinrichtung, so daß eine signifikante Störung der Grundstömung in diesem Bereich ausgeschlossen werden kann. Zur Aufnahme einer Druckverteilung mußte das Traversiergerät allerdings stets in seine unterste Position gefahren werden, da es in der Hitzdrahtmeßposition genau über den Druckanbohrungen stand.

\subsection{Grenzschichtströmung}

Die sich über dem Profil ausbildende Grenzschichtströmung ist dreidimensional (siehe Skizze des Geschwindigkeitsprofils im Stromlinienkoordinatensystem in Abbildung 3.1). Sie kommt durch die Änderung der Querströmung, ausgelöst vom Ungleichgewicht zwischen 


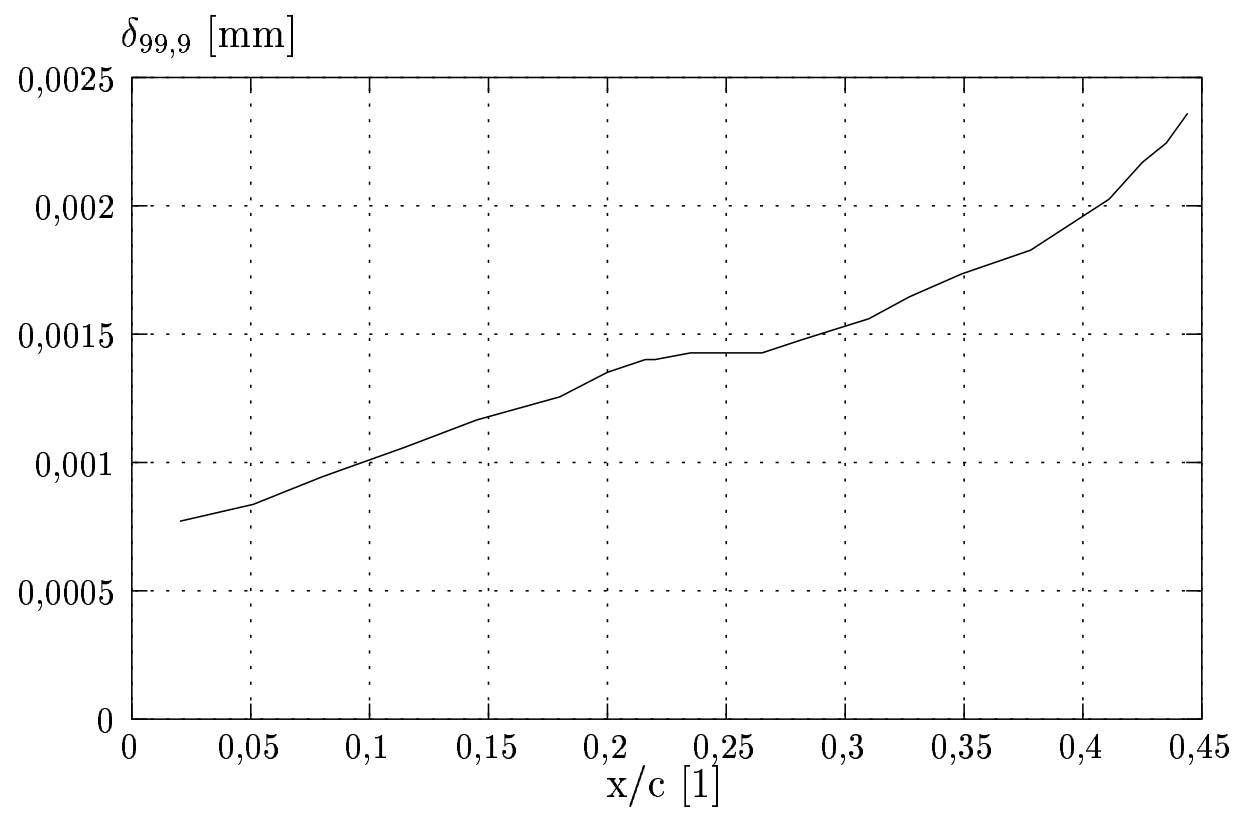

Abbildung 3.4: Entwicklung von $\delta_{99,9}$ in Abhängigkeit der Profiltiefe für $U_{\infty}=28 \mathrm{~m} / \mathrm{s}$ aus Grenzschichtrechnungen

Druck- und Zentrifugalkräften in der Grenzschicht, zustande: Der zur Vorderkante orthogonale Druckgradient und die Konstanz der Druckes in Spannweitenrichtung (siehe Gleichungen 3.3, 3.4) krümmen der Stromlinie am Grenzschichtrand. Das dort herrschende Gleichgewicht aus Druck- und Zentrifugalkräften prägt der Grenzschicht seinen Druck auf. In der Grenzschicht ist durch die geringere Geschwindigkeit dort auch die Zentrifugalkraft geringer. Das bisherige Kräftegleichgewicht ist gestört, und es kommt zur Ausbildung einer Sekundärströmung normal zur Stromlinie am Grenzschrichtrand. Diese Sekundärströmung nennt man Querströmung. Sie macht die Grenzschichtströmung auch bei unendlich schiebenden Bedingungen quasi dreidimensional.

Die Grenzschicht bleibt zunächst, bedingt durch den negativen Druckgradient, der die Strömung in Profiloberflächennähe beschleunigt, dünn (siehe Abb. 3.4).

Ab etwa 35\% Prozent Profiltiefe ändert der Druckgradient $d p / d x_{c}$ das Vorzeichen. Die Grenzschicht wird schnell dicker, und die Querströmung beginnt, die Richtung zu ändern (vgl. unterste Darstellung von $\left\langle V_{s}>\right.$ in Abbildung 3.6 auf Seite 31).

Ab etwa halber Profiltiefe kann es zu Grenzschichtablösung kommen, sofern die Grenzschicht an dieser Profiltiefe noch laminar ist. In diesem Fall ist der Impulsverlust innerhalb der Grenzschicht so stark, daß der Druckgradient in Strömungsrichtung nicht mehr überwunden werden kann. Es kommt zur Ausbildung einer Rückströmung. Ist die Grenzschicht an dieser Stelle jedoch bereits turbulent, so verzögert der größere Impulsaustausch der turbulenten Grenzschicht mit der Außenströmung dieses Ablösen und kann es unter Umständen ganz verhindern. 


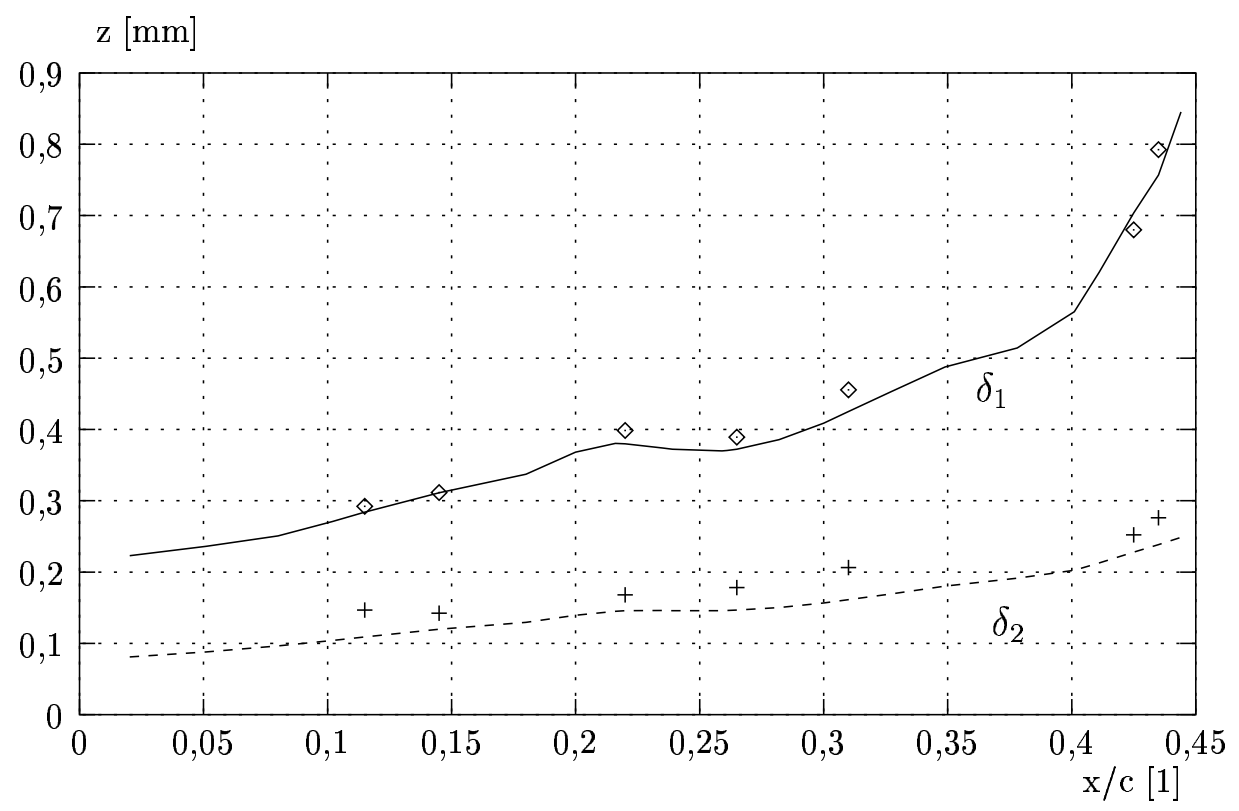

Abbildung 3.5: Entwicklung von $\delta_{1}$ und $\delta_{2}$ in Abhängigkeit der Profiltiefe für $U_{\infty}=28 \mathrm{~m} / \mathrm{s}$ aus Grenzschichtrechnungen (Linien) und den Experimenten (Symbole), $U_{\infty}=28 \mathrm{~m} / \mathrm{s}$

$\mathrm{Zu}$ den Messungen im Rahmen der hier vorgestellten Untersuchungen sollten begleitende numerische Berechnungen wie zum Beispiel Instabilitätsanalysen, durchgeführt werden. Deren Grundlage bildet eine möglichst exakte Reproduktion der Grundströmungssituation. Auf einen Vergleich zwischen experimentell ermittelten $\delta_{99,9 \%}$-Dicken und den aus den Grenzschichtrechnungen stammenden wurde verzichtet, da sie aus Gründen der Meßgenauigkeit experimentell nur ungenau bestimmt werden kann.

Für einen Vergleich besser geeignet ist die Betrachtung der Verdrängungsdicke $\delta_{1}$ bzw. der Impulsverlustdicke $\delta_{2}$, da sie als integrale Größen genauer aus den Messungen bestimmt werden können:

$$
\begin{array}{r}
\delta_{1}:=\int_{0}^{\infty}\left(1-\frac{U_{s}}{U_{(s, e)}}\right) d z \\
\delta_{2}:=\int_{0}^{\infty} \frac{U_{s}}{U_{(s, e)}}\left(1-\frac{U_{s}}{U_{(s, e)}}\right) d z
\end{array}
$$

Sie sind integrale Maße der Grenzschicht und hängen somit schwächer von dem asymptotisch in die Potentialströmung übergehenden Ast der Grenzschicht ab. Bei der Definition beider Größen wurde hier nur die $U_{s}$-Komponente der Geschwindigkeit berücksichtigt, da $V_{s}$, laut Definition des Stromlinienkoordinatensystems, am Rand verschwindet.

Diese Vergleiche sind in Abbildung 3.5 dargestellt. Man kann, zumindest für die Verdrängungsdicke eine sehr gute Übereinstimmung von Rechnung und Experiment feststellen. Die numerische Impulsverlustdicke wird allerdings stets kleiner berechnet, als die experimentell ermittelte. 
Die Grundströmung ist prinzipiell nicht direkt meßbar, da ihr in instabilen Grenzschichten stets Störungen überlagert sind. Im Bereich linearer Approximation kann aber der durch Instabilitäten hervorgerufene Anteil des Störgehalts durch spannweitiges und zeitliches Mitteln eleminiert werden. Dieses Verfahren wurde in Abbildung 3.6 angewandt. Darüber hinaus wurde bei den gemessenen Profilen der durch nicht exakte Justierung enstandene unterschiedliche Abstand der beiden Hitzdrähte zur Modelloberfläche nachträglich korrigiert, da er sonst die gemessene Querströmungskomponente stark beeinflußt. Die Korrektur lag im Bereich von $\pm 0,03 \mathrm{~mm}$.

Gemessene und berechnete Grenzschichtströmungen (siehe Abb. 3.6) zeigen ihre größten Abweichungen in den wandnahen Bereichen der $\left\langle U_{s}>\right.$-Komponente aufgrund der hohen Geschwindigkeitsgradienten an dieser Stelle und der extremen Wandnähe (Wandabstand $<0,2 \mathrm{~mm}$ ). In den übrigen Bereichen wurde eine gute Übereinstimmung von gemessener und berechneter Grundströmung gefunden, sieht man einmal von der größten Profiltiefe ab: Bei $x_{c} / c=0,425$ unterscheiden sich sowohl die $\left\langle U_{s}\right\rangle$ - als auch $\left\langle V_{s}\right\rangle$ - Komponente. Das $\left\langle U_{s}\right\rangle$-Profil ist im unteren Bereich steiler, im oberen flacher. $\left\langle V_{s}\right\rangle$ ist fülliger und nimmt sein Extremum bei einem kleineren Wandabstand an. An dieser Profiltiefenposition ist die lineare Approximation nicht mehr zulässig. Die instabilitätsbedingten Störungen treten in Wechselwirkung mit der Grundströmung und verändern auch das mittlere Geschwindigkeitsfeld, durch die Ausbildung der in der Literatur als $(0,0)$ - Mode bezeichneten Störung mit der Frequenz 0 und der Wellenzahl 0. 

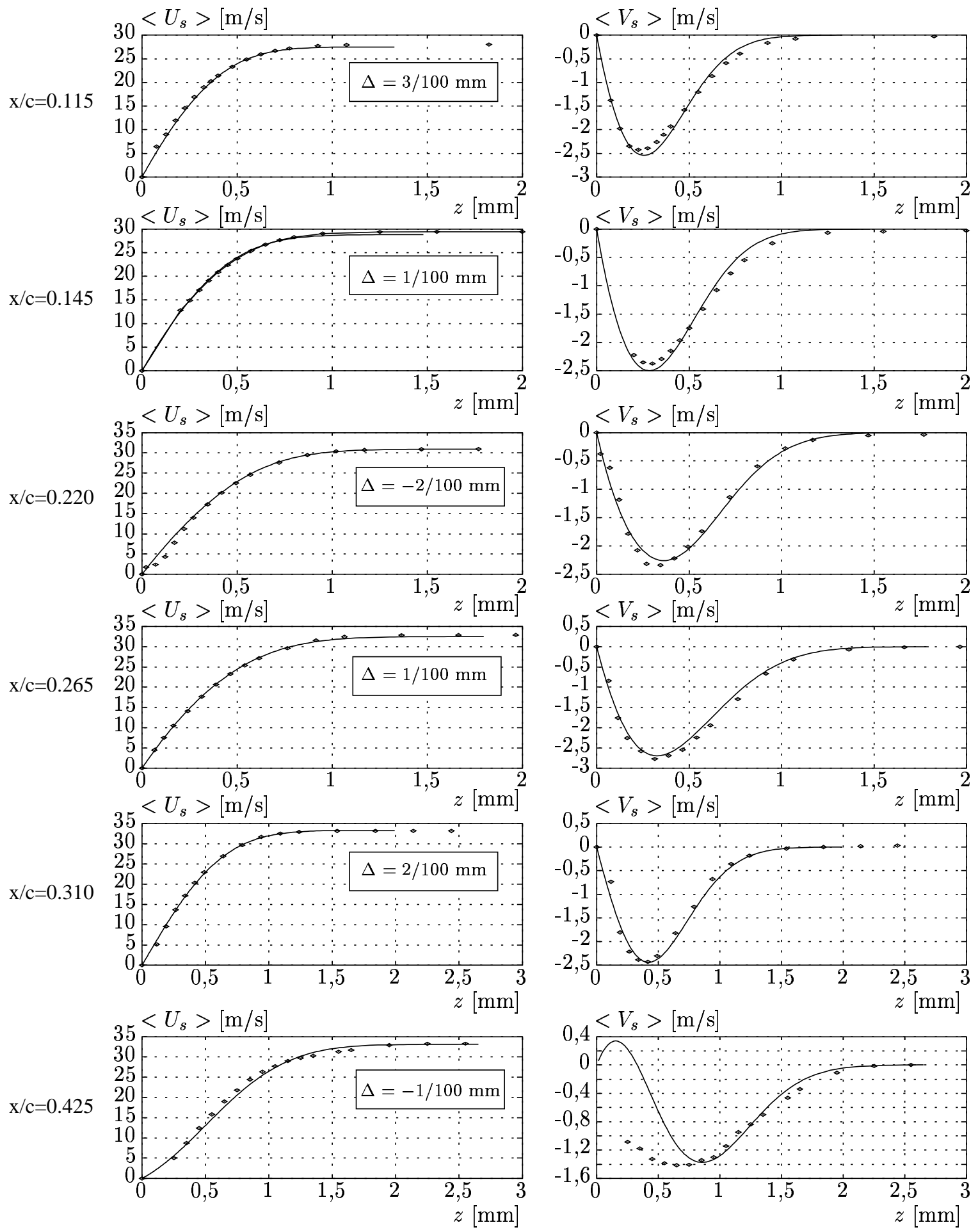

Abbildung 3.6: Vergleich der gemessenen, zeitlich und spannweitig gemittelten Strömung ( $<U_{s}>$ und $<V_{s}>$ ) (Symbole) mit der aus der Druckverteilung numerisch rekonstruierten Grenzschichtströmung (Linie) für verschiedene Profiltiefenpositionen. $\left(U_{\infty}=28 \mathrm{~m} / \mathrm{s}, \Delta\right.$ : Wandabstandskorrektur.) 


\section{Kapitel 4}

\section{Lineare Störungsentwicklung in der Grenzschicht}

Störungen der Grenzschicht werden über Instabilitäten selektiv verstärkt. Dieser Mechanismus ist für kleine Störungen verstanden und numerisch über die Verfahren der Stabilitätsanalyse von Grenzschichten gut handhabbar.

Der Mechanismus, mit dem diese Störungen in aus Oberflächeninhomogenitäten oder Anströmungsgeschwindigkeitsschwankungen die Grenzschicht einkoppeln (Stichwort Rezeptivität), ist gegenwärtig noch weitgehend ungeklärt. Untersuchungen zu diesem Thema gestalten sich schwierig, da die Störungen am Ort des Einkoppelns zu klein sind, um meßtechnisch aufgelöst zu werden. Aus diesem Grund lassen sich die Einkoppelprozesse kaum direkt beobachten. Erst in größerer Entfernung stromab, nachdem die Instabilitätsmechanismen gewissermaßen als Verstärker gewirkt haben, erreichen sie meßbare Größe. Nun kann man mit Kenntnis der Anströmungsstörungen und der Übertragungsfunktion der Grenzschicht auf die beteiligten Prozesse in der Umgebung der neutralen Linie schließen ${ }^{1}$. Infolgedessen beschränken sich die Messungen auf die bereits ausgebildeten Instabilitäten.

Es wurden bereits einige Experimente zur Transitionsbeschreibung beschleunigter dreidimensionaler Grenzschichtströmungen unternommen[37]. Aus Gründen der mathematischen Behandelbarkeit beschränken sich diese auf Strömungen, die innerhalb der Grenzen experimenteller Realisierbarkeit keine Gradienten in Spannweitenrichtung haben und auf Oberflächen vernachläßigbarer Krümmung (Unterseiten von Tragflügeln, ebene Platten mit aufgeprägtem Druckgradient).

\footnotetext{
${ }^{1}$ Unter dem Begriff der neutralen Linie versteht man die geometrischen Orte auf dem Flügelprofil, ab denen Störungen anwachsen. Gemäß linearer Stabilitätstheorie werden Störungen stromauf der neutralen Linie exponentiell gedämpft und ab dieser Linie erstmals ein Teil des möglichen Störspektrums exponentiell angefacht. Bei der zweidimensionalen Potentialströmung um das in guter Näherung unendlich schiebende Flügelprofil verläuft die neutrale Linie parallel zu Vorderkante.
} 


\subsection{Die lineare Stabilitätsanalyse}

Die lineare Stabilitätsanalyse untersucht das Problem, ob eine gegebene Grenzschichtströmung gegen kleine Störungen instabil werden kann. Falls die Störung anwächst, ist die Strömung gegen die betrachtete Störung instabil, anderenfalls stabil. Eine Einführung in das Gebiet geben Betchov, Criminale[41] und Drazin, Reid [42].

Die betrachtete Grundströmung $(\vec{Q}(\vec{x}), P(x))$, die quasi zweidimensionale Grenzschichtströmung, wie sie in Kapitel 3 beschrieben wurde, sei eine Lösung der inkompressiblen, stationären Navier-Stokes-Gleichungen.

Ihr wird eine instationäre Störströmung $(\vec{q}(\vec{x}, t), p(\vec{x}, t))$ überlagert, so daß die gestörte Grundströmung den instationären Navier-Stokes-Gleichungen genügt. Die Differenz der instationären und stationären Navier-Stokes-Gleichungen führt auf die (nichtlinearen) Störungsdifferentialgleichungen

$$
\begin{aligned}
\nabla \vec{q} & =0 \\
\frac{\partial \vec{q}}{\partial t}-\frac{1}{R e} \nabla^{2} \vec{q}+(\vec{q} \nabla) \vec{Q}+(\vec{Q} \nabla) \vec{q}+\nabla p & =(\vec{q} \nabla) \vec{q} .
\end{aligned}
$$

Werden in diesen Gleichungen alle in $\vec{q}$ nichtlinearen Glieder vernachlässigt, so ergeben sich die linearisierten Störungsdifferentialgleichungen, die, zusammen mit der Randbedingung abklingender Störungen an den Rändern der Grenzschicht, ein Anfangs-Randwertproblem darstellen. (Details siehe Anhang „Lineare Störungsentwicklung in der Grenzschicht“ auf Seite 97 sowie in Bieler [43] und in Hein et al. [44]).

Vernachlässigt man lokale und zeitliche Änderungen der Grundströmung in Stromabrichtung (Parallelströmungsannahme)

$$
\vec{Q} \equiv(U, V, 0)
$$

und Spannweitenrichtung (quasi zweidimensionale Strömung) und treten desweiteren keine äußeren Kräfte wie Zentrifugalkräfte infolge von Oberflächenkrümmungen, Corioliskräfte oder Auftriebskräfte auf, so reduziert sich das Lösen des partiellen Störungsdifferentialgleichungssystem auf das Lösen eines Systems gewöhnlicher Differentialgleichungen, dessen Koeffizienten nur noch von der Höhe in der Grenzschicht abhängen.

Macht man für dieses System einen Normalmodenansatz ${ }^{2}$, der wellenartige Störungen beschreibt,

$$
\overrightarrow{q_{0}}(\vec{x}, t) \equiv \hat{q}_{0}(z) \cdot e^{i(\vec{k} \vec{x}-\omega t)}
$$

mit

$$
\vec{k} \equiv(\alpha, \beta),
$$

$\left(\hat{q_{0}}, \vec{k}, \omega \in C\right)$ und setzt diesen in das System gewöhnlicher Differentialgleichungen ein, so erhält man die Orr-Sommerfeld-Squire-Gleichungen und überführt das AnfangsRandwertproblem mit der Haftbedingung auf der Tragflügeloberfläche in ein Eigenwertproblem.

\footnotetext{
${ }^{2}$ Eine Mode, deren Amplitude nur eine wandnormale Abhängigkeit hat und deren Phase sich wandparallel und in der Zeit ändert.
} 
Zusätzlich zum Spektrum diskreter Moden existiert in Grenzschichtströmungen ein kontinuierliches Spektrum, dessen Modenamplituden außerhalb der Grenzschicht nicht abklingen. Diese Moden sind allerdings stets stabil und werden deshalb nicht weiter betrachtet (Grosch und Salwen [45]) und mit einer zusätzlichen Randbedingung

$$
\vec{q}(z \rightarrow \infty) \equiv \overrightarrow{0}
$$

ausgeschlossen.

Unter Vernachlässigung dieses kontinuierlichen Spektrums besteht, bei gegebener Grundströmung und Re-Zahl, die Lösungsmenge der Orr-Sommerfeld-Squire-Gleichungen aus einem diskreten Spektrum endlich vieler Eigenwerte $(\alpha, \beta, \omega)$ mit den zugehörenden Eigenfunktionen. Von den vorhandenen diskreten Moden spielt für das Einsetzen der Instabilität nur die Fundamental-Mode eine Rolle. Diese ist durch die größte räumliche Anfachung $\sigma$ innerhalb des Modenspektrums gekennzeichnet,

$$
\sigma=\operatorname{Re}\left\{\frac{1}{q_{0}} \frac{\partial q_{0}}{\partial x}\right\}
$$

Die Betrachtung des dreidimensionalen Grenzschichtgeschwindigkeitsprofiles am schiebenden Tragflügel (siehe Abbildung 3.1 auf Seite 23) zeigt, daß die Geschwindigkeitskomponente in Querrichtung $\left(y_{s}\right)$ und in den dazu benachbarten Richtungen einen Wendepunkt aufweist. Nach Rayleigh [2] sind Wendepunkte eine notwendige Bedingung und für Grenzschichtströmungen nach Tollmien [3] auch eine hinreichende Bedingung für Instabilität, vorbehaltlich der stabilisierenden Wirkung der Zähigkeit. Darüber hinaus hat Mack [12] gezeigt, daß die Entwicklung der Instabilitätswellen durch die Geschwindigkeitskomponenten in Richtung ihrer Ausbreitung bestimmt wird. Danach führt die Querströmung zu einer Wendepunktinstabilität, der sogenannten Querströmungsinstabilität. Die beschleunigte Strömung wirkt in diesem Falle nicht stabilisierend, im Gegensatz zu Strömungssituationen ohne Wendepunktsprofile, in denen die Grenzschichten durch den Einfluß der Zähigkeit instabil werden können (Tollmien-Schlichting Instabilität). Somit wird der beschleunigte Bereich der Flügelumströmung dominiert durch die Querströmungsinstabilität während der verzögerte Bereich der Umströmung, stromab des Druckminimums auf der Saugseite des Tragflügels, erfahrungsgemäß durch die Tollmien-Schlichting Instabilität bestimmt wird.

Die Lösungen der Orr-Sommerfeld Gleichungen machen für Parallelströmungen eine Aussage über die zu einer vorgegeben Strömungssituation an einem Ort gefährlichsten Störungen und deren Anfachungen an diesem Ort. Für Grenzschichtströmungen, die von $x_{c}$ und $y_{c}$ abhängen, gelten ihre Aussagen somit allenfalls lokal ${ }^{3}$. Die Verbindung zwischen den Lösungen an verschiedenen Orten geht verloren, da die Parallelströmungsannahme die gleichen Lösungen für alle $\left(x_{c}, y_{c}\right)$ impliziert. Außerdem bleiben sowohl die äußeren Störungen als auch die Vorgeschichte der Störungen unberücksichtigt. Vergleiche mit experimentellen Resultaten sind daher nur beschränkt sinnvoll.

\footnotetext{
${ }^{3}$ Theorien, die durch Parallelströmungsannahme die Entwicklung der Grenzschicht und die Stromlinienkrümmung vernachlässigen, bezeichnet man daher als lokale Theorien.
} 
Eine bessere Grundlage für die Stabilitätsanalyse von Grenzschichtströmungen stellen die parabolisierten Stabilitätsgleichungen (PSE, vgl. Herbert, Bertolotti [17]) dar. Auf sie führt das Einsetzen des im Vergleich zu Gleichung (4.4) allgemeineren Störungsansatzes

$$
\vec{q}(\vec{x}, t) \equiv \hat{q}_{0}(\epsilon x, \epsilon y, z) \cdot e^{i \Theta(\vec{x}, t)},
$$

mit $\epsilon=O(1 / R e), \omega=\partial \Theta / \partial t$ und

$$
\vec{k}(\epsilon x, \epsilon y)=(\alpha, \beta)=\nabla \Theta
$$

in die Störungsdifferentialgleichungen (4.1) sowie der Verzicht auf die Parallelströmungsannahme durch die Formulierung der Grundströmung in der Form

$$
\vec{Q} \equiv(U, V, \epsilon W)
$$

Das resultierende Gleichungssystem stellt in Verbindung mit den Anfangsbedingungen

- vorgegebene Werte für $R e, \beta, \omega, \vec{Q}$

- geschätzte Werte für den Eigenwert $\alpha_{0}$ und die Eigenfunktion $q_{0}$ am Ort $\left(x_{0}, y_{0}\right)$

ein Anfangs-Randwertproblem dar, dessen Randwerte sowohl homogen als auch inhomogen vorgegeben werden können.

Dieses Verfahren bietet den Vorteil, daß man durch geeignete Wahl des Ortes $\left(x_{0}, y_{0}\right)$ die Anfangswerte der Störungen in der Rechnung denen eines Experiments angleichen kann, um quantitative Vergleiche durchzuführen. Vor allem aber sind mit dem PSE-Verfahren Schnittstellen vorhanden, die Umwelteinflüsse eines Experiments über ein Rezeptivitätsmodell als inhomogene Randwerte in die Rechnungen einfließen zu lassen. Der möglich gewordene Verzicht auf die Parallelströmungsannahme sowie die Stromabvariabilität der Wellenzahl der betrachteten Störung ermöglichen die Berücksichtigung der sich stromab verändernden Grenzschicht sowie der Entwicklungsgeschichte einer Störung.

Ein weiterer Vorteil dieses Verfahrens besteht in der Möglichkeit der konsistenten Berücksichtigung der Wirkung der Terme der Oberflächenkrümmung auf die Stabilitätseigenschaften. Um die Wirkung der Oberflächenkrümmung von den Effekten der Stromlinienkrümmung zu unterscheiden (die Stromlinienkrümmung findet sich in der Ebene parallel zur Oberfläche, die Oberflächenkrümmung normal dazu), formuliert man das System der Navier - Stokes Gleichungen in ein an die Stromlinie am Grenzschichtrand angepasstes krummliniges orthogonales Koordinatensystem, wie dies beispielsweise in Malik et al. [56] ausgeführt ist. Die sich dabei ergebenden Oberflächenkrümmungsterme sind typischerweise von gleicher Ordnung wie die Terme der Nichtparallelität der Strömung und werden daher in der klassischen Orr-Sommerfeld Theorie konsequenterweise, genau wie die Nichtparallelität, vernachlässigt.

Eine wesentliche Einschränkung erfährt das PSE-Verfahren durch die Beschränkung auf in Stromabrichtung nur langsam veränderliche Grundströmungen. In Bereichen großer 
Nichtparallelität der Strömung, wie im Nasenbereich eines Tragflügels, ist diese Näherung verletzt. Da diese Bereiche den Ausgangspunkt für die weitere Störungsentwicklung liefern, versucht man gegenwärtig, diese Bereiche mit alternativen Modellen zu behandeln. In der vorliegenden Untersuchung wird dieser Bereich nicht betrachtet.

\subsection{Begrifflichkeiten}

Generell ist die Verwendung der Bezeichnung "Mode“ im Zusammenhang mit experimentellen Ergebnissen in dieser Arbeit diskussionswürdig, da in Experimenten unter natürlichen Bedingungen stets eine Überlagerung aller vorhandenen räumlichen und zeitlichen Störkomponenten am Meßort aufgenommen wird,

$$
\vec{u}(\vec{x}, \omega)=\Sigma_{\vec{k}} \hat{u}(\vec{x}, \vec{k}, \omega) \cdot e^{i(\vec{k} \vec{x}-\omega t)} .
$$

Darüber hinaus beschränken sich die detektierten Störungen nicht nur auf ebene Wellen oder Wellenpakete als Resultat der Wirkung von Instabilitäten, sondern beinhalten ebenso die lokale Reaktion der Grenzschicht auf räumlich und zeitlich zufällig auftretende Inhomogenitäten der Umgebung.

Ich werde deshalb zur Beschreibung experimenteller Ergebnisse auf den allgemeineren Begriff „Störung“ ausweichen. Wenn von Moden im Sinne der linearen Stabilitätstheorie die Rede ist, so wird das durch Verwendung des Symbols $q$ für die Geschwindigkeitsfluktuation deutlich gemacht.

\subsection{Die Entwicklung ortsfester Wirbel}

Die Stabilitätsanalysen für den Bereich beschleunigter Grenzschicht ergaben neben den Lösungen mit $\alpha, \beta, \omega \neq 0$ auch die Existenz von Lösungen mit $\omega=0$. Diese Lösungen beschreiben ortsfeste Wirbel innerhalb der Grenzschicht und werden als stationäre Moden bezeichnet. Im Falle der Querströmungsinstabilität sind diese Moden von besonderer Bedeutung. Sie weisen zwar nicht unbedingt die größten Anfachungen auf, werden aber im Vergleich zu den laufenden Moden $(\omega \neq 0)$ selbst unter Freiflugbedingungen mit großen Anfangsamplituden angeregt und erreichen damit frühzeitiger signifikante Amplituden (Deyhle,Bippes [34]). Die Ursache dafür liegt in dem Prozeß des Einkoppelns von Störungen, die aus den Fluktuationen in der Anströmung resultieren oder solchen, die durch Oberflächenrauhigkeiten verursacht werden. Letztere wirken direkt, d.h. ohne Umskalierprozesse bedingt durch Unterschiede in der Wellenlängen (Crouch [55]).

\subsubsection{Identifikation stationärer Störungen}

Im folgenden sollen zunächst die im Experiment auftretenden Störungen identifiziert und mit denen aus den Vorhersagen der Stabilitätsanalysen verglichen werden. 


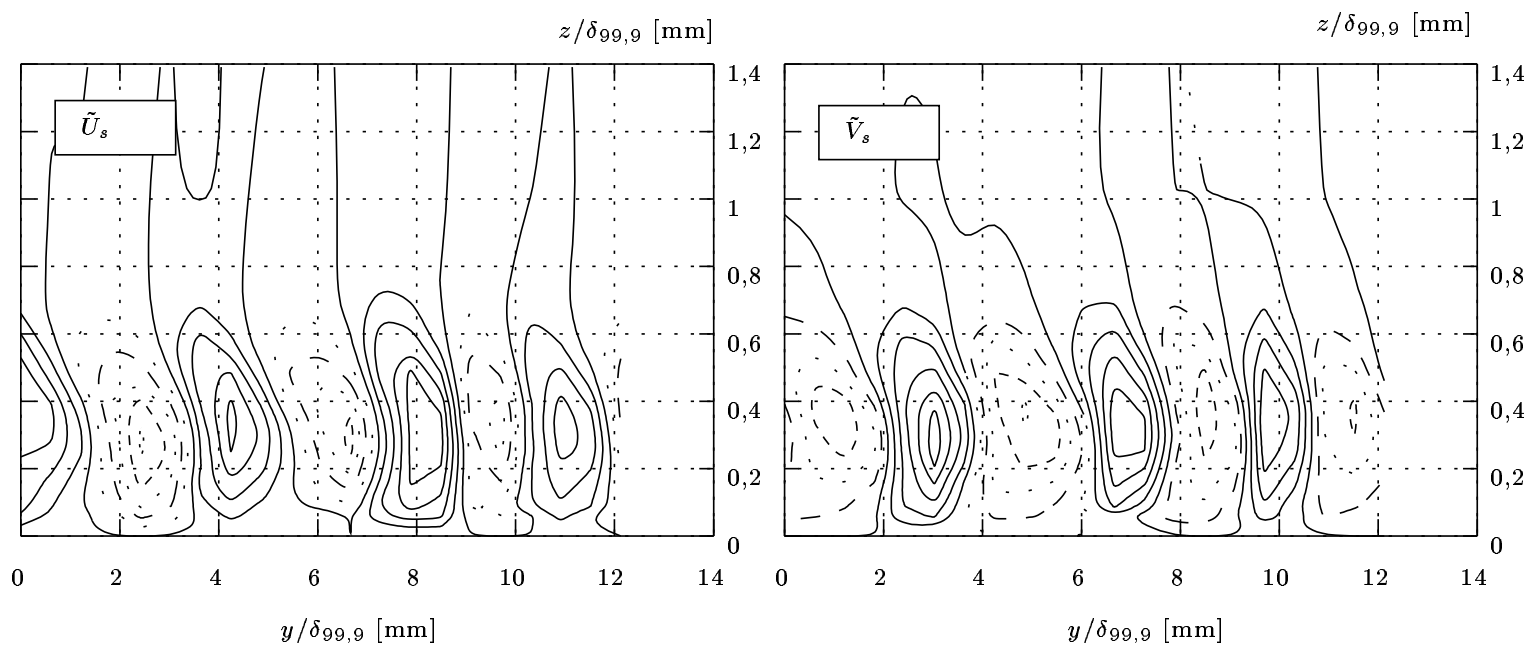

Abbildung 4.1: Isotachen der stationären Geschwindigkeitsfluktuationen $\tilde{U}_{s} / Q_{e}, \tilde{V}_{s} / Q_{e}$. Linienabstand 1\%, durchgezogene Linien entsprechen Geschwindigkeiten $\leq 0, x / c=0,326$, $\delta_{99,9}=1,65 \mathrm{~mm}$

Ortsfeste Wirbel sind charakterisiert durch das zeitlich gemittelte Geschwindigkeitsfeld $\vec{U}(x, y, z)$. Dieses bestimmt man, indem bei $x_{c}=$ const. entlang der Spannweite eine Reihe zeitlich gemittelter Grenzschichtprofile aufgenommen werden. Das Geschwindigkeitsfeld $\tilde{\overrightarrow{U_{s}}}$ mit

$$
\tilde{\vec{U}}_{s}=\overline{\vec{U}}_{s}-<\vec{U}_{s}>
$$

bezeichnet die spannweitige Modulation des zeitlich und spannweitig gemittelten Grenzschichtprofils $\left\langle\vec{U}_{s}\right\rangle$. In Abbildung 4.1 ist es beispielhaft dargestellt. Das Geschwindigkeitsfeld stellt gewissermaßen die Summe aller vorhandenen stationären Instabilitätswellen dar,

$$
\tilde{\vec{U}}_{s}=\Sigma_{(\alpha, \beta, 0)} q_{0}
$$

solange die Annahme linearer Störungsüberlagerung gerechtfertigt ist und man somit $<\vec{U}_{s}>$ als ungestörte Grundströmung interpretieren kann. $\tilde{\vec{U}}_{s}$ entwickelt sich aus einer anfangs spannweitig konstanten mittleren Geschwindigkeitskomponente, die stromab zunehmend in ihrer Amplitude modelliert wird.

Da die Orientierung der Achsen der stationären Querströmungswirbel im Wesentlichen mit der Richtung der Stromlinie am Grenzschichtrand zusammenfällt, d.h.

$$
|\alpha| \approx 0
$$

genügt es, die spannweitige Wellenlänge bzw. die Querwellenzahl $\beta$ zu bestimmen, um die stationären Querströmungswirbel zu charakterisieren. Mit Hilfe einer räumlichen Fourieranalyse des spannweitig zeitlich gemittelten Geschwindigkeitsfeldes kann man den Betrag 

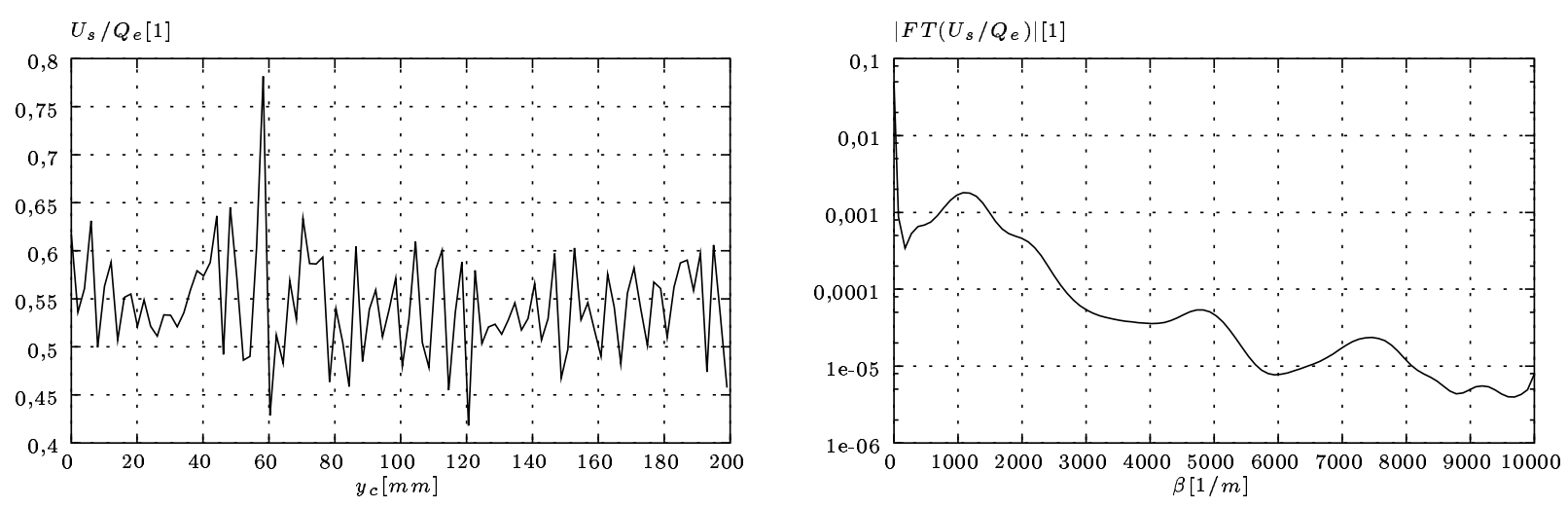

Abbildung 4.2: Ermittlung der Wellenzahlen und der zugehörigen Amplituden stationärer Störungen, links: Spannweitige Variation der mittleren Geschwindigkeit $U_{s}$ in der Grenzschicht bei $z_{0}=0,5 \mathrm{~mm}, x_{c} / c=0,4, Q_{e}=44 \mathrm{~m} / \mathrm{s}$, rechts: Fouriertransformierte, geglättet.

der Wellenzahlkomponente $\beta$ darstellen ${ }^{4}$ (Abbildung 4.2). Für spannweitige Wellenlänge $\lambda_{y_{c}}$ und Querwellenzahl $\beta$ gilt:

$$
\lambda_{y_{c}}=\frac{2 \pi}{|\beta|}
$$

Das Ergebnis in Abbildung 4.2 (rechts) macht deutlich, daß im Bereich $\beta=1000 / \mathrm{m}$ die stationären Querströmungswirbel größter Amplitude gefunden werden. Geht man von breitbandiger Anregung stationäerer Störungen aus, so kann man folgern, daß Störungen mit zugehöriger Querwellenzahl besonders stark angefacht werden.

\subsubsection{Präparation eines Feldes homogener stationärer Störungen}

Experimente unter natürlichen Bedingungen haben den Nachteil, oftmals keine reproduzierbaren Strömungsfelder über tagelange Meßkampagnen hinweg zu garantieren. Um Probleme wie die Störungsentwicklung bearbeiten zu können, ist es aber notwendig, stets dieselben Störungen in ihrer Stromabentwicklung verfolgen zu können. Ein Hilfsmittel ist es, Störungen bestimmter Art gezielt anzuregen, und zwar mit einer Anfangsamplitude, die größer ist als diejenigen, die aus dem Störgehalt der Umgebung entstehen. Auf diese Weise heben sie sich aus den natürlichen Störungen heraus und können in ihrer Stromabentwicklung beobachtet werden.

Einzelne Rauhigkeitselemente in der Nähe der neutralen Linie regen modellfest stehende stationäre Wirbel an. Vorderkantenparallel periodisch angebrachte Rauhigkeitselemente

\footnotetext{
${ }^{4}$ Legt man den Normalmodenansatz zu Grunde, dann genügt es, für eine definierte Position in der Grenzschicht eine Traversenmessung aufzunehmen und die gemessenen Geschwindigkeitsabhängigkeit von $\left.y_{c}, \vec{U}_{s}\left(x_{(} c, 0\right), y_{c}, z_{0}\right)$ zu fouriertransformieren. Zweckmäßigerweise wählt man dafür $z_{0}$ so, daß man sich am Ort des Maximums der Amplitudenfunktion der spannweitigen Modulation befindet.
} 
generieren ein reproduzierbares regelmäßiges Feld stationärer Wirbel, deren Entwicklung man stromab verfolgen kann. In gewissen Grenzen ist man mit dieser Methode frei, bestimmte spannweitige Wellenlängen vorzugeben (siehe Abbildung 4.3). Je weniger allerdings die vorgegebene Wellenlänge mit der sich auch unter natürlichen Bedingungen in Folge ihrer hohen Anfachung als dominant herausstellenden spannweitigen Wellenlänge der am meisten angefachten Mode übereinstimmt, desto ungleichmäßiger wird das Feld der generierten stationären Wirbel in der weiteren Entwicklung. Desweiteren hat man darauf zu achten, daß man nicht gegen eventuell bereits vorhandene natürliche Störungen auf dem Profil anregt, da sonst ebenfalls eine über die Spannweite unregelmäßig verteilte Wirbelamplitude die Folge ist (siehe Abbildung 4.4). Wählt man Wellenlängen und „Phase“ der Stimulation passend, so genügen bereits periodische Rauhigkeiten von ca. $10 \mu m$ Höhe und einem Durchmesser von $d=1,5 \mathrm{~mm}$, um ein regelmäßiges Feld stationärer Wirbel zu generieren. Dabei ist es nicht nötig, jeden stationären Wirbel zu stimulieren, wie die Abbildung 4.5 zeigt.

Im Gegensatz zu dreidimensionalen Grenzschichten an ebenen und konkav gekrümmten Wänden, die im selben Kanal untersucht wurden, stellte sich die letztlich dominante Wellenlänge im Experiment als durch Stimulation nicht beeinflußbar heraus (siehe Abbildung 4.6). Die Wellenlänge der letztlich dominanten stationären Störung zeigt gute Übereinstimmung mit der, nach linearer lokaler Theorie am meisten angefachten Störung, wie im nächsten Abschnitt gezeigt wird.

Es gelang, mit dem Mittel der Stimulation stationärer Störungen ein Gebiet ortsfester stationärer Wirbel zu schaffen, das als Grundlage der Untersuchungen zur Stromabentwicklung von Störungen diente. Für alle weiteren Darstellungen wurde eine spannweitig periodische Stimulation von der Wellenlänge $\lambda_{y_{(c, s t)}}=6 \mathrm{~mm}$ und der Höhe $20 \mu \mathrm{m}$ in einer Entfernung von $\Delta x_{c}=9 \mathrm{~mm}$ stromab der neutralen Linie gewählt. Damit gelang es, eine reproduzierbare Störströmung zu schaffen, die es ermöglichte, durch zeitlich aufeinanderfolgende Messungen die Stromabentwicklung der stationären Wirbel bei gleichen Anfangsbedingungen zu verfolgen, das heißt die Anfachung für quantitative Vergleiche mit Rechnungen zu bestimmen.

\subsubsection{Stromabentwicklung verschiedener stationärer Störungen}

Das Wachstum einer Instabilitätswelle $q_{0}(\alpha, \beta, 0)$ beginnt ab der zu ihrem Wellenzahlvektor $\vec{k}$ gehörenden neutralen Linie. Instabilitäten gegen stationäre Störungen treten in den numerischen Untersuchungen erstmals ab einer Profiltiefenposition von $x / c=0,048$ auf, wie man in Abbildung 4.7 erkennt $\left(U_{\infty}=28 \mathrm{~m} / \mathrm{s}\right.$, Schiebewinkel $\Phi=45^{\circ}$, Anstellwinkel $\alpha=-3,5^{\circ}$ ). Dabei zeigen die betrachteten Moden im Bereich 0,048 $\leq x_{c} / c \leq 0,075$ eine zunächst relativ schwache Anfachung. Als am meisten angefachte stationäre Wellenlänge wurde $\lambda_{y_{c}}=5 \mathrm{~mm}$ ermittelt, wobei die Unterschiede zu den benachbarten Wellenlängen $\lambda_{y_{c}}=4 \mathrm{~mm}$ und $\lambda_{y_{c}}=6 \mathrm{~mm}$ klein sind. In Abbildung 4.8 sind die experimentellen Ergebnisse der Entwicklung von Störungen verschiedener spannweitiger Wellenlängen dargestellt 

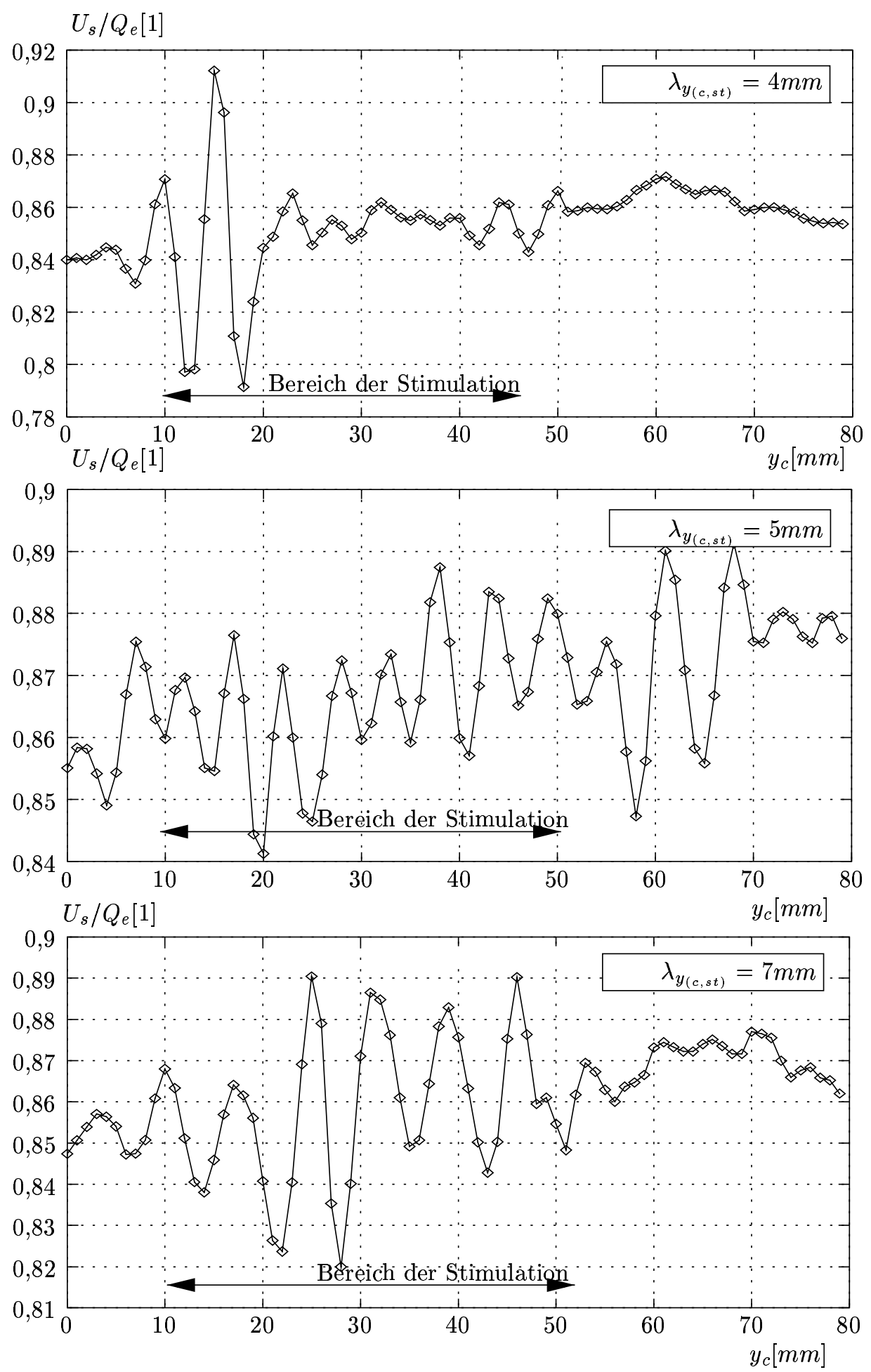

Abbildung 4.3: Beeinflussung der spannweitigen Wellenlänge durch spannweitig periodische Rauhigkeitselemente bei $x / c=0,05, U_{\infty}=28 \mathrm{~m} / \mathrm{s}$, Meßort bei $x / c=0,217$ 


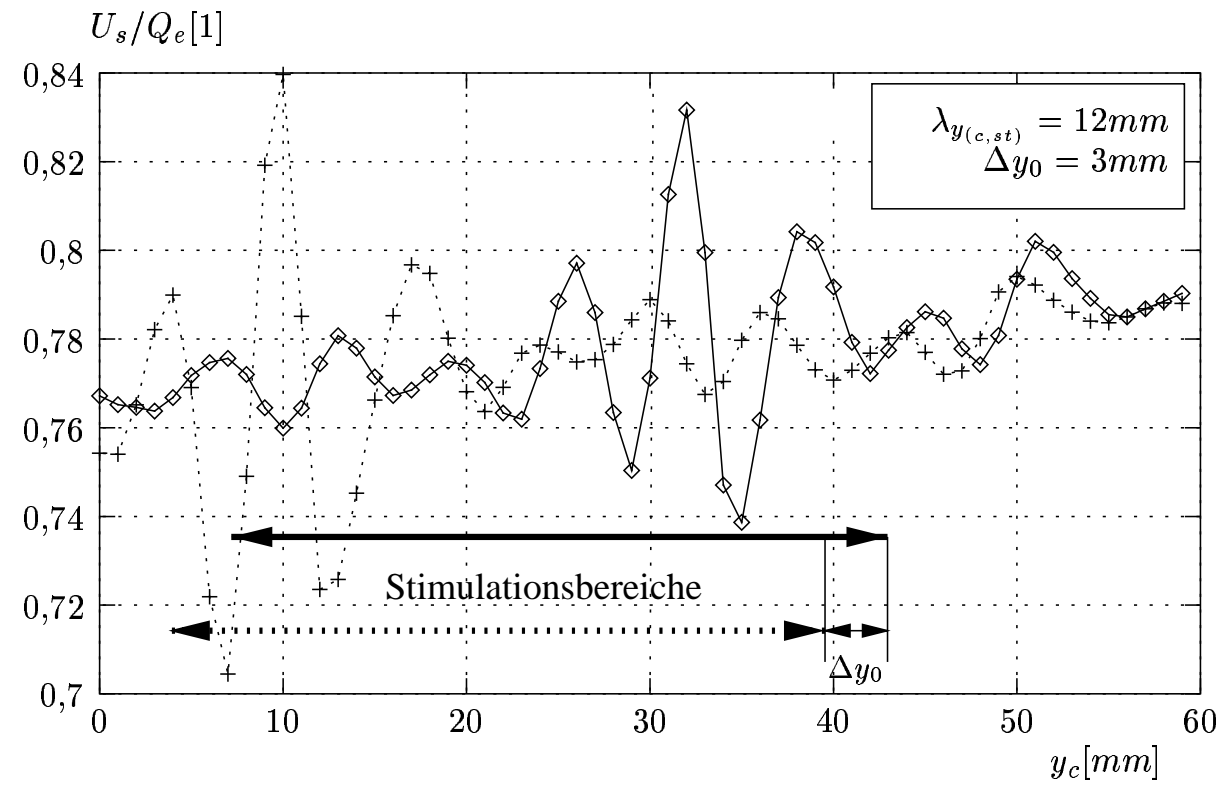

Abbildung 4.4: Interaktion spannweitig periodischer Rauhigkeitselemente mit Profilinhomogenitäten, $U_{\infty}=28 \mathrm{~m} / \mathrm{s}$, Meßort bei $x / c=0,217$

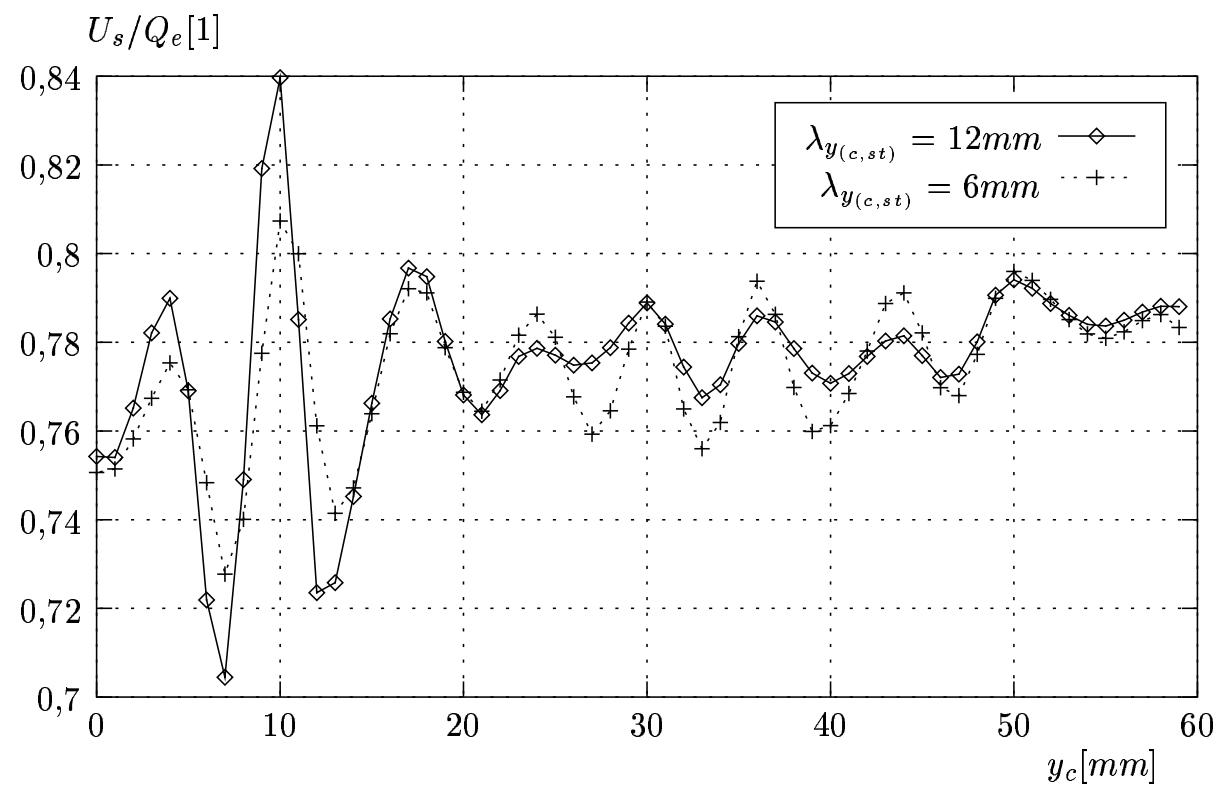

Abbildung 4.5: Vergleich der Anregung jedes stationären Wirbels mit der Anregung jeden zweiten Wirbels, $U_{\infty}=28 \mathrm{~m} / \mathrm{s}$, Meßort bei $x / c=0,217$ 


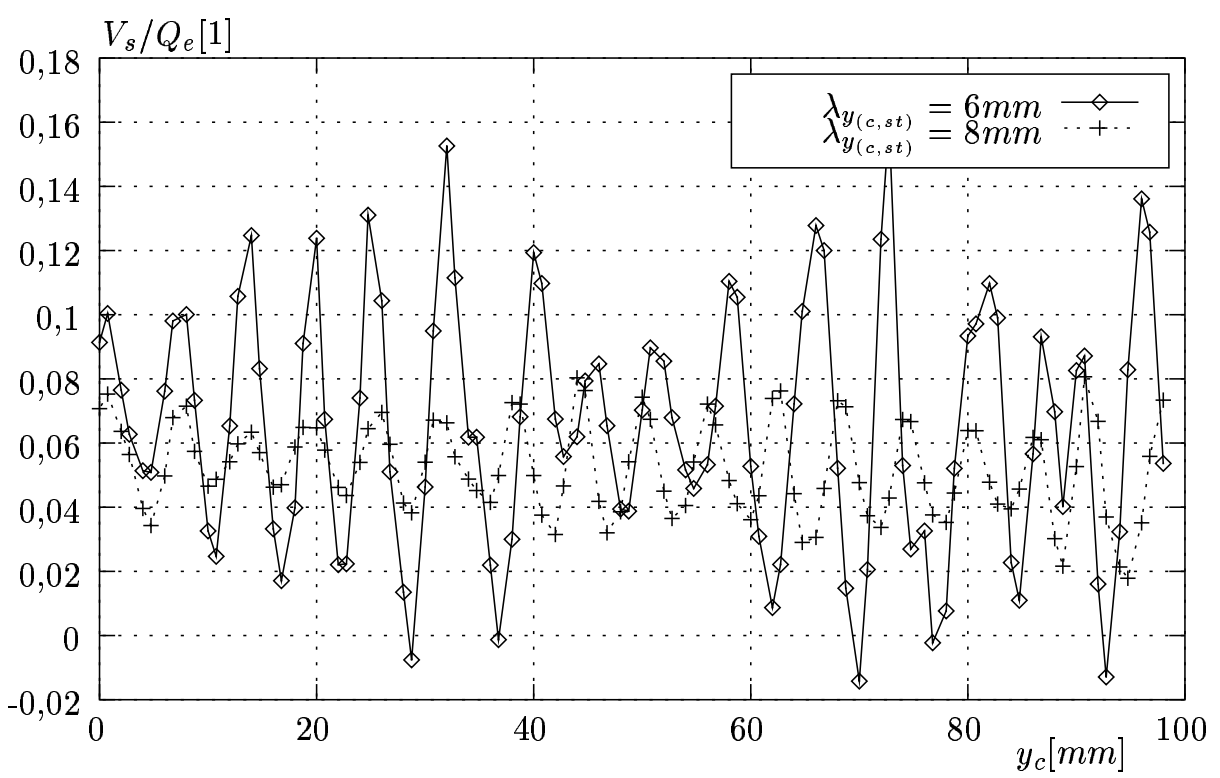

Abbildung 4.6: Stimulierung verschiedener spannweitiger Wellenlängen für den Meßort $x / c=0,261, U_{\infty}=28 \mathrm{~m} / \mathrm{s}$

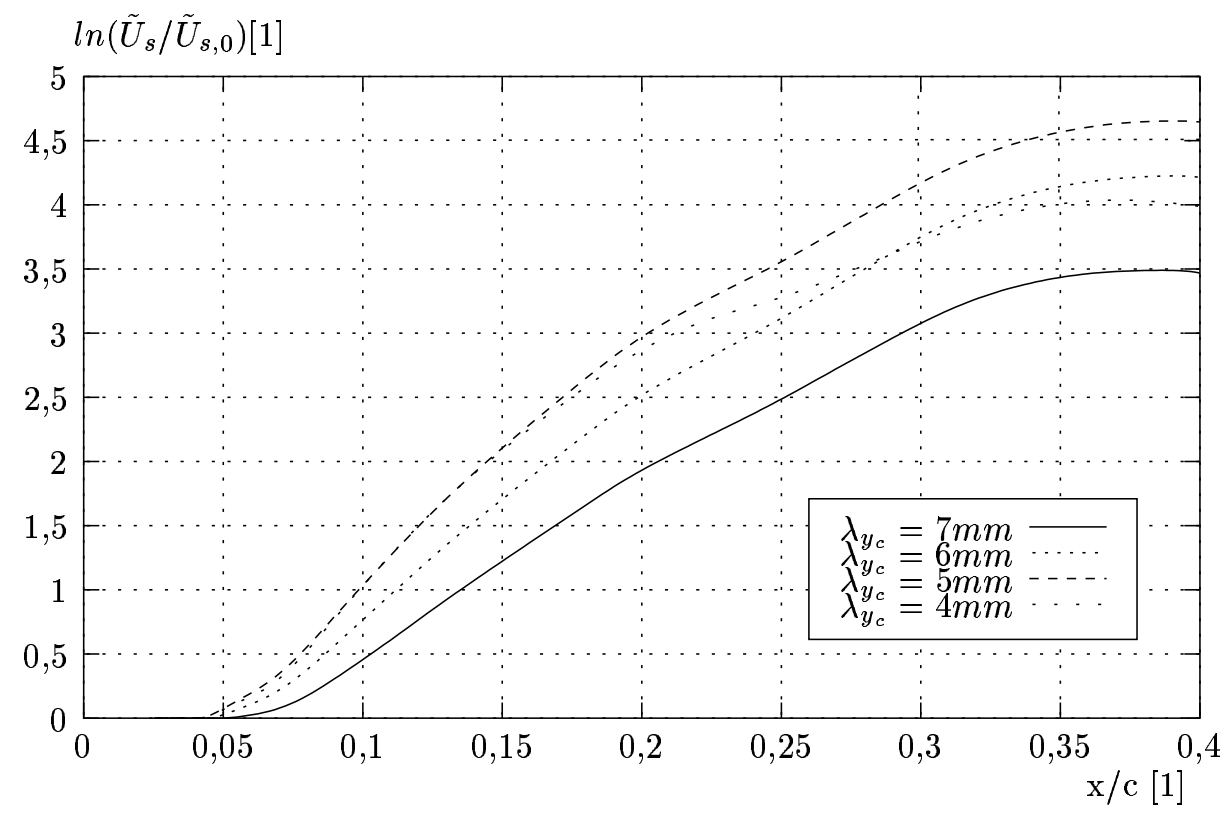

Abbildung 4.7: Entwicklung stationärer Störungen verschiedener spannweitiger Wellenlänge gemäß linearer Instabilitätsanalyse, $U_{\infty}=28 \mathrm{~m} / \mathrm{s}$, Rechnungen v. Hein [46] mit linearem lokalem Verfahren unter Berücksichtigung von Oberflächenkrümmungseffekten 


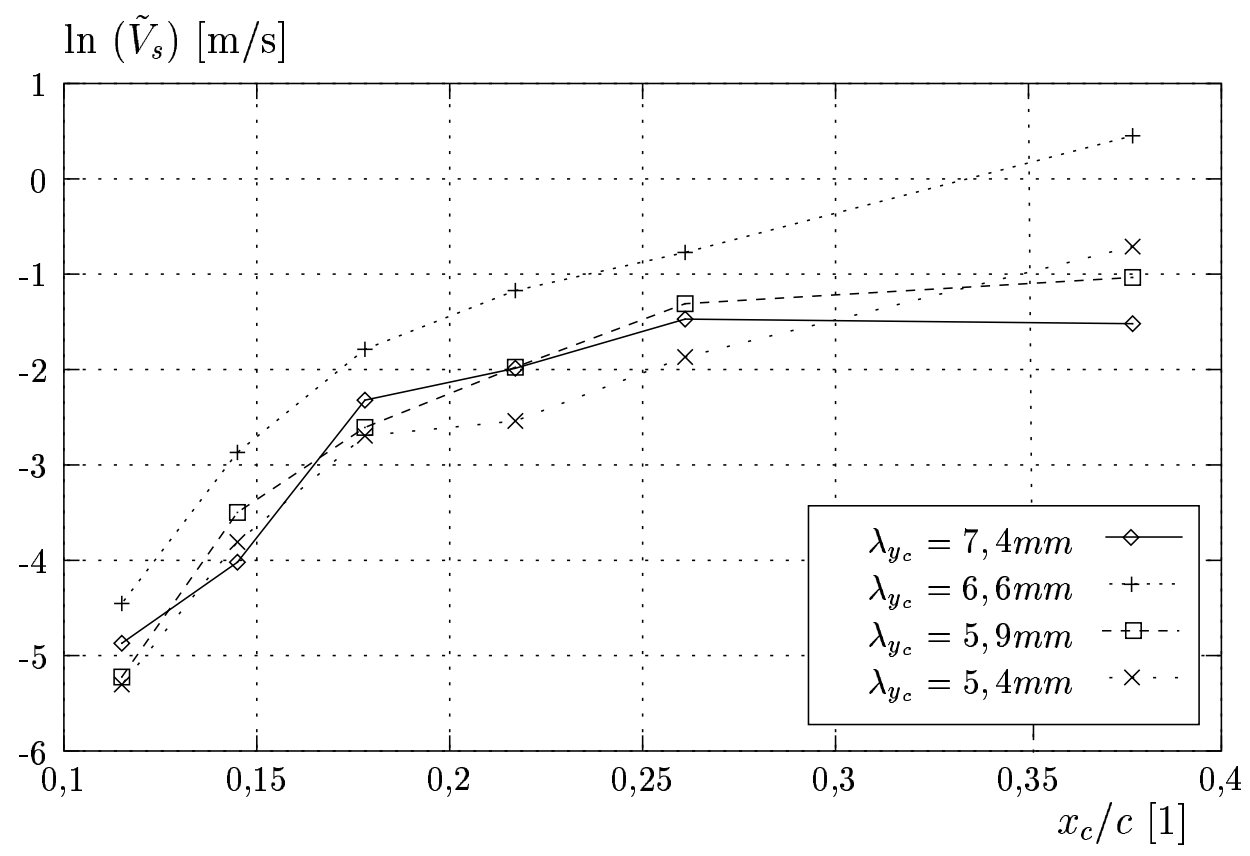

Abbildung 4.8: Experimentell ermittelte Stromabentwicklung der stationären Querströmungswirbel verschiedener spannweitiger Wellenlängen

$\left(U_{\infty}=28 m / s\right.$, Schiebewinkel $\Phi=45^{\circ}$, Anstellwinkel $\left.\alpha=-3,5^{\circ}\right)$. Dieser Darstellung liegen Traversenmessungen über große spannweitige Bereiche bei verschiedenen Profiltiefenpositionen zugrunde, die nicht notwendigerweise eine regelmäßige Modulation des zeitlich gemittelten Geschwindigkeitsfeldes beinhalten. Ziel dieser Darstellung ist es, das Anwachsen verschiedener Wellenlängen gegenüberzustellen.

Die Messungen zeigen für die erste Meßposition bei $x_{c} / c=0,12$ bereits starke Unterschiede in der Amplitude der dargestellten Querwellenlängen. Die Wellenlänge $\lambda_{y_{c}}=$ $6,6 \mathrm{~mm}$ besitzt die größte Amplitude. Sie wächst stromab am meisten und erreicht an der letzten untersuchten $x_{c} / c$-Position auch die größten Amplitudenwerte. Bemerkenswert ist dabei die Tatsache, daß nicht die stimulierte Wellenlänge $(6 \mathrm{~mm})$ die größten Amplituden aufweist. Das läßt die Vermutung zu, daß die Reihe von Stimulationspunkten nicht exakt parallel zur neutralen Linie verlief. Durch die starke Krümmung der Stromlinien in der Umgebung der Reihe der Stimulationspunkte kann sich dadurch eine andere effektive Stimulationswellenlänge ergeben.

Die Entwicklung der spannweitigen Spektralkomponenten verläuft über einen weiten Bereich parallel. Erst ab einer Profiltiefe von $x_{c} / c=0,25$ übertrifft das Wachstum der 6,6 $\mathrm{mm}$ - Komponente diejenigen der anderen Wellenzahlkomponenten.

Die Unterschiede zwischen der experimentell ermittelten am meisten angefachten Querwellenlänge $\lambda_{y_{c}}=6,6 \mathrm{~mm}$ und der numerisch vorhergesagten von $\lambda_{y_{c}}=5 \mathrm{~mm}$ sind klein. Es genügen bereits geringe Unterschiede zwischen der der Stabilitätsanalyse zugrunde liegenden Grundströmung und der im Experiment real vorhandenen, um diese Differenzen zu 


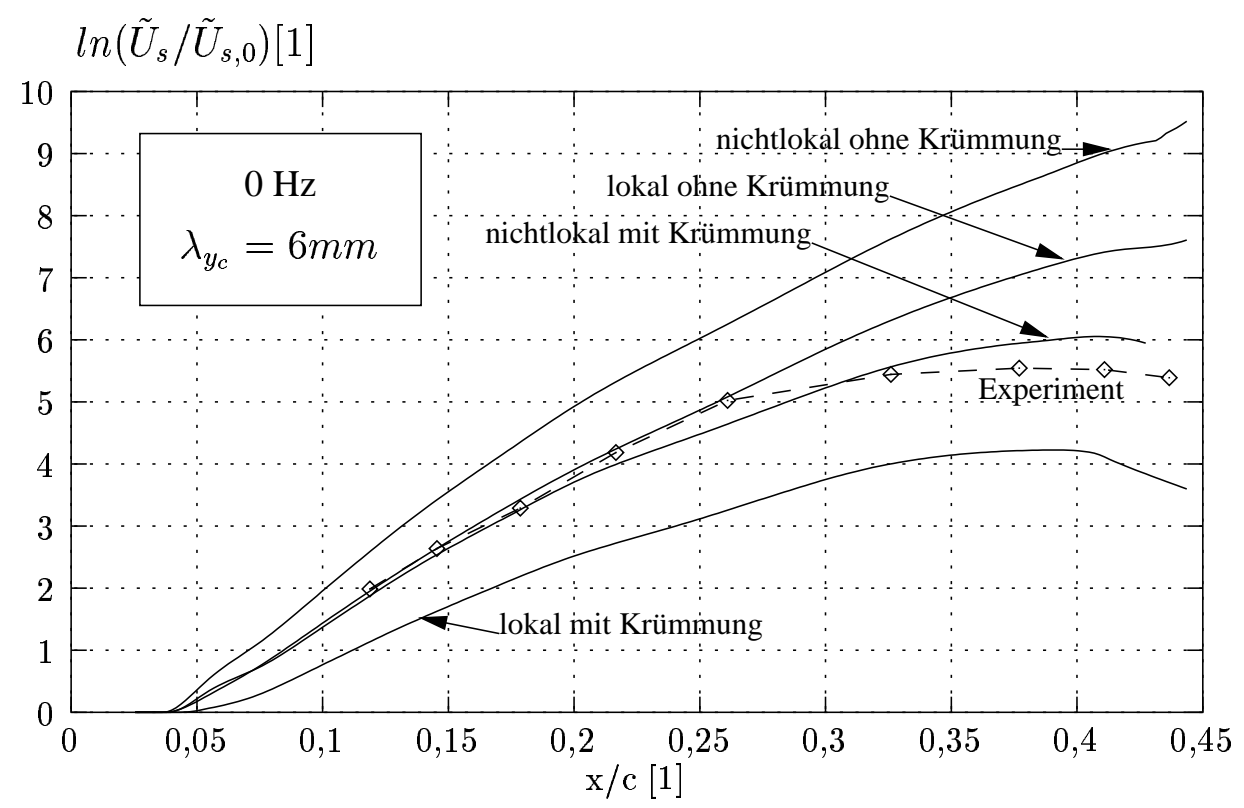

Abbildung 4.9: Vergleich von numerischer und experimentell ermittelter relativer Störungsamplitudenentwicklung für stationäre Störkomponenten $\tilde{U}_{s}$ der Querwellenlänge $\lambda_{y_{c}}=$ $6 \mathrm{~mm}$. Berechnungen von Hein [46] mit linearem NOLOT/PSE [57]

verursachen. Der Bereich der angefachten Störungen konnte mit guter Qualität numerisch reproduziert werden.

Die der Abbildung 4.8 zugrunde liegenden Messungen umfaßten Traversenmessungen über einen weiten Bereich und viele Querströmungswirbel. Im weiteren dient nun ein engerer Bereich aus fünf benachbarten Querströmungswirbeln, der auf Grund großer Regelmäßigkeit $\left(\lambda_{y_{c}}=6 \mathrm{~mm}\right)$ und Reproduzierbarkeit ausgewählt wurde und sich deshalb auch gut mit den Ergebnissen der linearen Stabilitätsanalysen vergleichen läßt, da diese nur eine isolierte Mode (in diesem Fall diejenige mit der spannweitigen Wellenlänge von $\left.\lambda_{y_{c}}=6 \mathrm{~mm}\right)$ in ihrer Entwicklung wiedergeben können.

\subsubsection{Entwicklung einer einzelnen dominanten Störung und der Einfluß der Oberflächenkrümmung}

In der Abbildung 4.9 ist die gemessene Amplitudenentwicklung einer stationären Störung der Querwellenlänge $\lambda_{y_{c}}=6 \mathrm{~mm}$ den Ergebnissen aus Rechnungen verschiedener linearer Theorien gegenübergestellt. Um den Krümmungseinfluß auf die vorliegende Strömungssituation herauszuarbeiten, wurden die Ergebnisse von lokalen und nichtlokalen Rechnungen jeweils mit und ohne Berücksichtigung der Krümmungsterme durchgeführt. Vergleicht man zunächst die gleichartigen Theorien mit und ohne Krümmungsterme miteinander, so stellt man fest, daß sowohl die lokalen als auch die nichtlokalen Rechnungen einen deut- 
lichen Stabilisierungseffekt gegenüber stationären Störungen durch die Berücksichtigung der Oberflächenkrümmung ermitteln. Falls sich dies als richtig herausstellt müssen die derzeit in der angewandten Entwurfsaerodynamik verwendeten Transitionskriterien angepaßt werden.

Die Berücksichtigung der Änderungen der Grenzschicht resultiert in einer stärkeren Anfachung der betrachteten Störung, d.h. die sich ändernde Grenzschicht hat einen destabilisierenden Effekt.

Um die experimentell ermittelte Störungsentwicklung mit den Ergebnissen der verschie-

denen linearen Theorien vergleichen zu können, wurden die experimentell ermittelten Störungsamplituden entsprechend normiert.

Der Vergleich offenbart, daß die in sich konsistenten Theorien, also lokal ohne Krümmungsterme bzw. nichtlokal mit Krümmung, die größte Übereinstimmung mit dem Experiment zeigen. Die nichtkonsistenten über- bzw. unterschätzen das Amplitudenwachstum klar.

Da alle dargestellten numerischen Ergebnisse mit einer linearisierenden Näherung durchgeführt wurden, können sie auch nicht das Abflachen des Wachstums bei $x_{c} / c=0,3$ wiedergeben. Hier sind die Störungsamplituden bereits so groß, daß eine lineare Theorie der Physik nicht mehr gerecht wird.

Der Vergleich zwischen Theorie und Experiment macht deutlich, daß die Oberflächenkrümmung einen signifikanten Einfluß auf die Entwicklung stationärer Störungen hat und bei der Voraussage der Transition unbedingt $\mathrm{zu}$ berücksichtigen ist. Die derzeit in der Praxis angewandten Verfahren sind entsprechend zu ändern.

\subsection{Die Entwicklung instationärer Störungen}

Unter instationären Störungen der Grenzschichtströmung versteht man zeitliche und räumliche Modulationen der Grenzschichtgeschwindigkeitskomponenten.

Ihr Ursprung sind zufällige, räumlich eng begrenzte instationäre Anströmungsinhomogenitäten oder Erschütterungen ebenso wie großräumig korrelierte Vibrationen oder Schall. Sie „koppeln“ zunächst in die Grenzschicht ein und beginnen, mit der sich weiterentwickelnden Grenzschichtströmung zu interagieren. In ihr regen sie Eigenstörungsmoden an. Für einige dieser Eigenstörungen existiert eine neutrale Linie, ab der sie angefacht sind, dh. ab der sie zunächst exponentiell anwachsen, während andere gedämpft bleiben (siehe Abbildung 4.17 auf Seite 55). In der weiteren Entwicklung dominieren die angefachten Störungen das vorhandene Störspektrum der laminaren Grenzschicht.

\subsubsection{Identifikation instationärer Störungen}

Prinzipiell gelten auch bei der experimentellen Identifikation instationärer Instabilitätsmoden die selben Einschränkungen, wie sie bereits im Abschnitt 4.2 auf Seite 37 dargelegt 


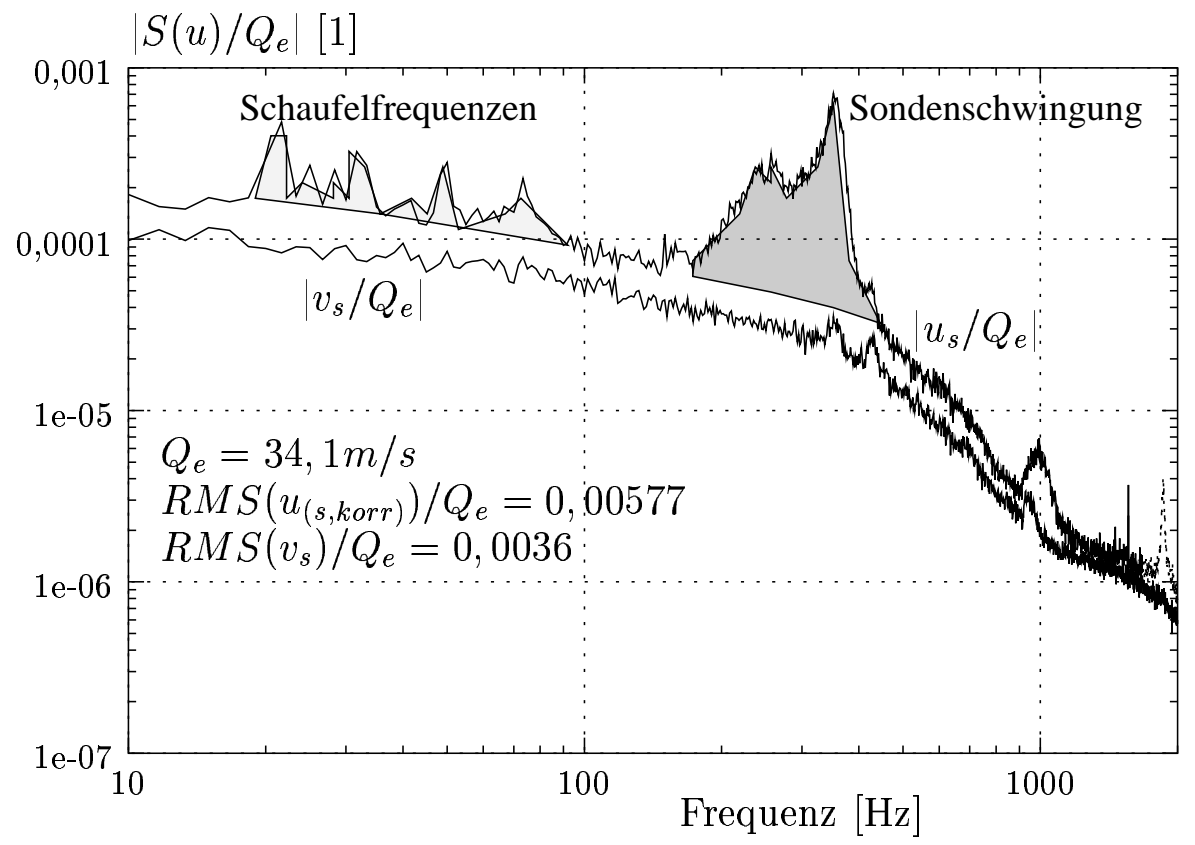

Abbildung 4.10: Betrag des normierten, spannweitig gemittelten Amplitudenspektrums von $u_{s}$ und $v_{s}$ bei $x_{c} / c=0,2166, Q_{e}=34,1 \mathrm{~m} / \mathrm{s}, U_{\infty}=28 \mathrm{~m} / \mathrm{s}$ und $\mathrm{z}=z_{\text {max-crossfow }}$

wurden. Gemessen werden keine diskreten Moden, sondern stets die Superposition des gesamten vorhandenen Störspektrums am Ort der Sonde.

Um trotzdem Aussagen über einzelne Bestandteile des Störspektrums zu machen, bleibt im wesentlichen die Anwendung der zeitlichen Fouriertransformation ${ }^{5}$ auf die Transienten der interessierenden physikalischen Größe. Damit separiert man zunächst die Störungen einer bestimmten Frequenz $\omega_{0}$ und betrachtet nun nur noch die Superposition aller Störungen

$$
\vec{u}\left(\vec{x}, \omega_{0}\right)=\Sigma_{\vec{k}} \hat{u}\left(\vec{x}, \vec{k}, \omega_{0}\right) \cdot e^{i\left(\vec{k} \vec{x}-\omega_{0} t\right)} .
$$

Abbildung 4.10 stellt ein spannweitig gemitteltes, auf die Grenzschichtrandgeschwindigkeit normiertes Amplitudenspektrum für die Geschwindigkeitskomponenten $u_{s}$ bzw. $v_{s}$ in der Höhe der maximalen Querkomponente $\left\langle V_{s}>\right.$ dar. Man erkennt zunächst die über einen weiten Frequenzbereich $10 \mathrm{~Hz}<f<2000 \mathrm{~Hz}$ vorhandenen instationären Störungen. Desweiteren ist $\left|u_{s}(f)\right|$ etwa $60 \%$ größer als $\left|v_{s}(f)\right|$. Die in Abbildung 4.10 schraffiert dargestellte Fläche bezeichnet die Frequenzbereiche, in denen Sondenschwingungen den Wert von $u_{s}$ verfälschen. Man kann an Hand der Sondenaufhängung davon ausgehen, daß die Sondenschwingungen in der durch $y_{s}$ und $z$ aufgespannten Ebene stattfinden, wobei die mittlere Auslenkung keine Vorzugsrichtung haben wird. Diese Bewegung hat zur Folge,

\footnotetext{
${ }^{5} \mathrm{Zu}$ Details des Standardverfahrens siehe zum Beispiel Luke [59]
} 


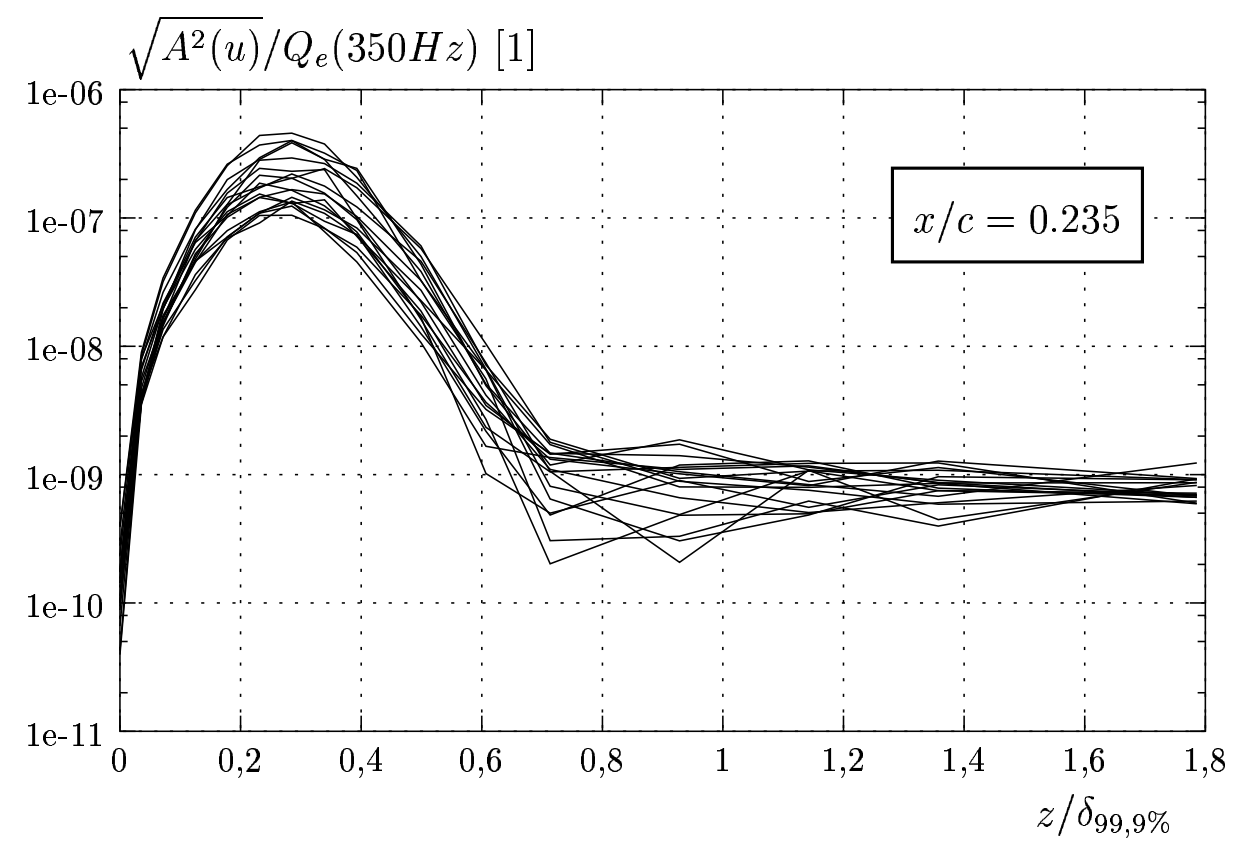

Abbildung 4.11: Experimentell bestimmter Betrag der Überlagerung aller Störungen zu einer Frequenz von $f=350 \mathrm{~Hz}$ für verschiedene Spannweitenpositionen. $Q_{e}=31 \mathrm{~m} / \mathrm{s}$, $U_{\infty}=28 \mathrm{~m} / \mathrm{s}$

daß sich die Sondenspitze, also der Teil, der die Meßwerte aufnimmt, im zeitlich gemittelten Geschwindigkeitsfeld bewegt. Dieses weißt besonders in der Nähe der Wand große Gradienten $\partial U_{s} / \partial z$ auf, weshalb in dieser Komponente im Bereich der Resonanzfrequenz der Sonde große Schwankungen gemessen werden. Diese Schwankungen übertreffen das natürlich vorhandene Störspektrum insbesondere an Stellen kleiner $x_{c} / c$, da dort einerseits das Störspektrum noch klein und andererseits die Grenzschicht noch dünn ist, also große wandnormale Gradienten der Hauptströmungskomponente existieren. Berechnet man, ausgehend von solchen offensichtlich stark gestörten Amplitudenspektren, die RMS-Werte

$$
R M S\left(u_{s}\right)=\sqrt{\frac{2}{T} \int_{0}^{f_{G}} S^{2}\left(u_{s}\right) d f}
$$

so wird man zu hohe Schwankungswerte berechnen. Da in erster Näherung $S\left(u_{s}\right)$ und $S\left(v_{s}\right)$ eine ähnliche Frequenzabhängigkeit aufweisen, kann man die bekannte Gestalt von $S\left(v_{s}\right)$ für die Berechnung eines die Sondenschwingungsamplituden korrigierenden $u_{s}$-Spektrums nutzen. Dieses Verfahren wurde bei der Ermittlung der korrigierten $\operatorname{RMS}\left(u_{s}\right)$-Werte in Abbildung 4.10 angewandt.

Eine exemplarische Amplitudenverteilung der Störungen der u-Komponente mit der Frequenz $f=350 \mathrm{~Hz}$ ist in Abbildung 4.11 dargestellt.

Um auch einzelne räumliche Komponenten laufender Instabilitätswellen zu untersuchen, wäre es nötig, die betrachtete Meßgröße mit der gewünschten räumlichen Auflösung 
aufzunehmen. Der meßtechnische Aufwand dafür wäre allerdings enorm und ist, aufgrund der Störung der Strömung durch die Vielzahl der dazu nötigen Sonden, auch nur mit berührungslosen Meßverfahren, wie z.B. PIV, möglich. Diese Verfahren haben allerdings noch Schwierigkeiten mit Messungen in dünnen Grenzschichten über gekrümmten Oberflächen und der ebenfalls nötigen hohen zeitlichen Auflösung.

Eine weitere Möglichkeit der gezielten Analyse des Verhaltens laufender Moden in der Grenzschicht stellt ihre gezielte Anregung dar, wie sie beispielsweise von Lerche [14] angewendet wurde. Diese Möglichkeit bot sich für das vorliegende Experiment nicht, da der Einbau der Anregung die Charakteristika des Modells stark verändert hätte.

Um trotzdem Aussagen über die räumlichen Parameter $(\alpha, \beta)$ der in der Grenzschicht vorhandenen Störungen treffen zu können, wurde das im Abschnitt 2.2.2 auf 19 geschilderte Verfahren angewandt, das es unter Verwendung einiger weiterer Annahmen zur Gestalt der Störungen gestattet, dem Summenvektor instationärer Störungen einer Frequenz $\omega_{0}$ eine Richtung zuzuordnen. Voraussetzung dieses Verfahrens ist allerdings eine ausreichend große spannweitige Kohärenz natürlicher Störungen.

\section{Spannweitige Kohärenz natürlicher Störungen}

Eine in der Literatur insbesondere durch die Arbeiten von Gaster aufgeworfene Frage ist die nach der spannweitigen Kohärenz von natürlichen Störungen. In seinen Experimenten untersucht er die Evolution von punktförmig in die Grenzschicht eingebrachten, quasi stochastischen Störungen und betrachtet ihre Entwicklung als Ausbildung und Ausbreitung von Wellenpaketen.

Untersuchungen zur Kohärenz von Störungen fehlen jedoch bisher für dreidimensionale Grenzschichten.

Zur Klärung dieser Frage führt man in der Grenzschicht für viele spannweitige Abstände Kohärenzuntersuchungen durch, das heißt, man bringt zwei Hitzdrahtsonden in einen definierten Abstand zueinander und an vergleichbare Postionen in der Grenzschicht $(x, z=$ const.).

Zur Durchführung der Untersuchungen zur Kohärenz natürlich vorhandener Störungen wurde eine Mehrfachhitzdrahtsonde (siehe Abb. 4.12 ) verwendet, die den hohen experimentellen Aufwand solcher Untersuchungen minimiert. Mit ihr war es möglich, mit vier Sonden und einer Messung Kohärenzen für sechs verschiedene spannweitige Abstände zu messen.

Ein Maß der Kohärenz ist die Kohärenzfunktion

$$
\gamma^{2}(f, x):=\frac{\left(S_{A}^{*} \cdot S_{B}\right)\left(S_{B}^{*} \cdot S_{A}\right)}{\left(S_{A}^{*} \cdot S_{A}\right)\left(S_{B}^{*} \cdot S_{B}\right)}
$$

mit

$$
S_{A}(f)=\mathcal{F}\left\{s_{A}(t)\right\}
$$

und $x:=$ Abstand $\mathrm{A} \leftrightarrow$ B. $s_{A}(t)$ sei das Geschwindigkeitssignal von Hitzdraht $A$. 


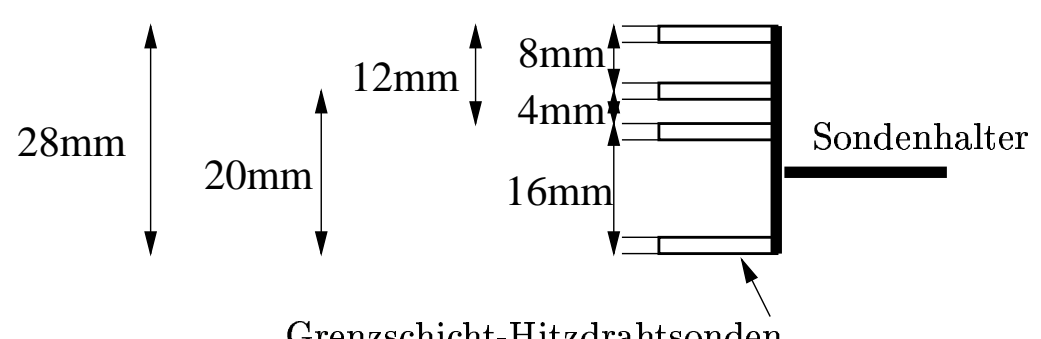

Grenzschicht-Hitzdrahtsonden

Abbildung 4.12: Schematische Darstellung der zur Kohärenzuntersuchung verwendeten Sonde. Die Abstandpfeile bezeichnen die gleichzeitig meßbaren Abstände.

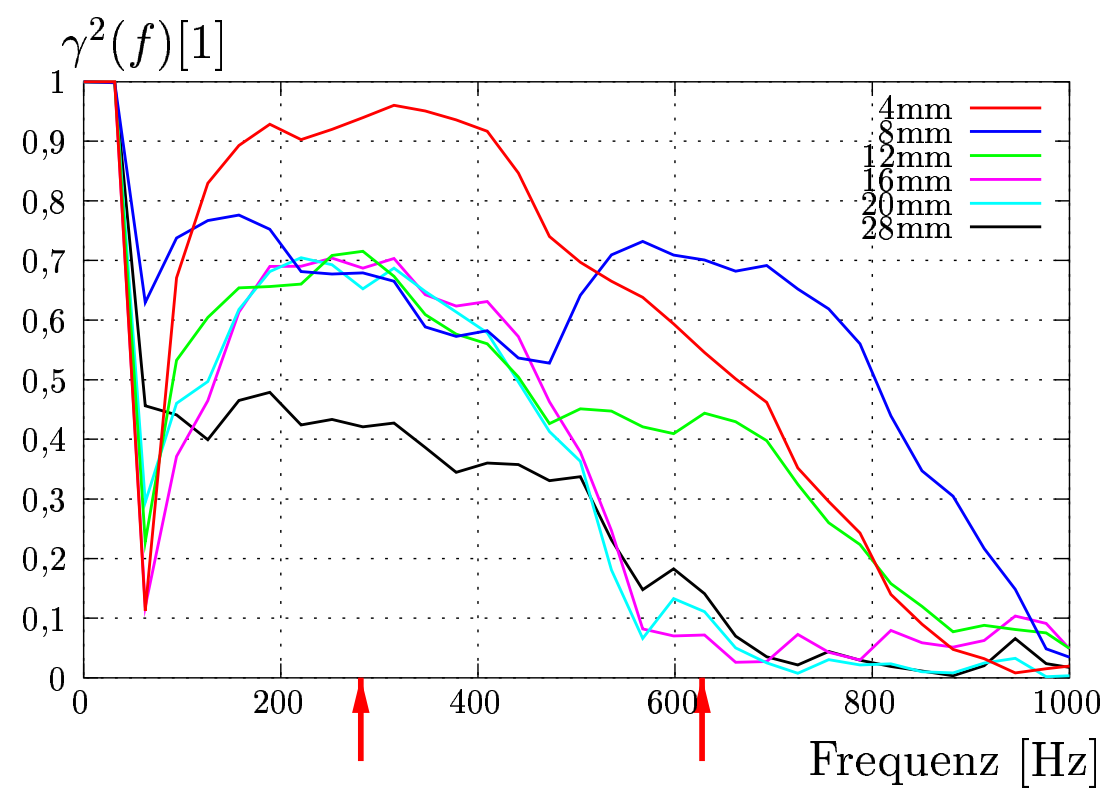

Abbildung 4.13: Kohärenzfunktion von $u_{s}$ für verschiedene spannweitige Abstände. $x / c=0,3$

Die Kohärenzfunktion ist damit ein mit den beiden Leistungsspektren der Einzelgeschwindigkeitssignale normiertes Kreuzspektrum. Spektralanteile, die nicht in konstanter Phasenlage zueinander sind, werden im Zuge der Kreuzspektrumsbildung zu Null. Die anschließende Normierung bildet das Ergebnis in das Intervall $0 \leq \gamma^{2}(f, x) \leq 1$ ab. Vollständig in Phase befindliche Frequenzkomponenten haben den Wert $\gamma^{2}=1$, stochastisch verteilte Phasen liefern den Wert $\gamma^{2}=0$.

Die Ergebnisse der Untersuchungen sind in Abbildung 4.13 dargestellt. Im Unterschied zu anderen Darstellungen in diesem Kapitel sind die Abstände der Sonden in Profilkoordinaten angegeben. Geschwindigkeitsschwankungen mit tiefer Frequenz $(f \leq 50 \mathrm{~Hz})$ sind für alle Abstände sehr gut kohärent. Das ist nicht verwunderlich, da in diesen Bereich sowohl die durch die Kanalsteuerung verursachten Schwankungen der Anströmungsgeschwindig- 
keit als auch die Schaufelfrequenzen des Antriebs die maßgeblichen Schwankungsursachen sind, und diese sind über den gesamten Kanalquerschnitt und somit auch in der Grenzschicht $\mathrm{zu}$ finden.

Ein weiteres Maximum der Kohärenzfunktion findet sich im Bereich der primär angefachten Störungen $(100 \mathrm{~Hz} \leq f \leq 500 \mathrm{~Hz})$. Der kleinste realisierte Abstand $(4 \mathrm{~mm})$ besitzt in diesem Frequenzbereich eine Kohärenz von 0,9 bis 0,95. Für größere Abstände sinkt dieser hohe Wert ab. Zunächst auf ein Plateau von etwa 0,7 für Abstände im Bereich $8 \mathrm{~mm} \leq y \leq 20 \mathrm{~mm}$. Der größte realisierte Abstand besitzt in diesem Frequenzbereich lediglich eine Kohärenz von 0,4 .

Normiert man die Abstände auf die spannweitig dominante Wellenlänge von $\lambda=6 \mathrm{~mm}$ findet man sehr gute Kohärenz innerhalb einer stationären Wellenlänge. Bis etwa vier bis fünf Wellenlängen bleibt die Kohärenz der primär angefachten Störungen immer noch hoch. Erst ab Abständen in der Größenordnung von sechs stationären Wirbeln sinkt die Kohärenz unter den Wert von 0,5. Diese Ergebnisse sind unabhängig von der spannweitigen Meßposition, d.h. unabhängig von der Sondenlage bezüglich der Phase stationärer Störungen auf dem Profil.

Für Frequenzen am oberen Rand des primär angefachten Störungsbereichs findet man hohe Kohärenzen für Störungen im Bereich benachbarter stationärer Wirbel. Für größere Abstände verschwindet diese Kohärenz allerdings rasch.

Zusammenfassend kann man sagen, die spannweitige Kohärenz von unter dem Einfluß von Querströmungsinstabilitäten natürlich entstandenen instationären Grenzschichtstörungen nimmt bereits über dem ersten stationären Wirbel zunächst stark ab, geht allerdings selbst über große spannweitige Abstände nicht verloren. Somit kann man davon ausgehen, daß die Modellvorstellung ebener Wellen in der Grenzschicht auch unter natürlichen Bedingungen die Realität gut beschreibt.

\section{Wellenlängen und Ausbreitungsrichtungen instationärer kohärenter Störungen}

Zur Identifizierung instationärer Querströmungsinstabilitätswellen müssen die Wellenzahlvektoren bestimmt werden. Entsprechend des in Abschnitt 2.2.2 auf Seite 19 dargestellten Verfahrens wurde für eine Profiltiefenposition von $x / c=0,3$ und die Anströmungsgeschwindigkeit $U_{\infty}=28 \mathrm{~m} / \mathrm{s}$ eine Bestimmung der instationären Wellenzahlvektoren durchgeführt. Die Ergebnisse sind in Abbildung 4.14 dargestellt.

Die in diesem Bereich nach linearer Theorie am meisten angefachte Mode besitzt einen Wellenvektor mit $\lambda=6,2 \mathrm{~mm}$ und einer Ausbreitungsrichtung von $\gamma=-79^{\circ}$ zur Stromlinie am Grenzschichtrand (vgl. Tabelle 4.1). Für einen Vergleich wurden in die Darstellung die numerischen Ergebnisse des zur entsprechenden Frequenz zugehörigen Wellenvektors mit der größten integralen Anfachung eingetragen, wie man ihn entlang der gestrichelten Linie in Abbildung 4.15 (und den korrespondierenden Werten für $\alpha_{S}$ in Abbildung 4.17) findet.

Der Vergleich in Abbildung 4.14 offenbart eine hinreichende Übereinstimmung im Bereich großer Störamplituden $(f \approx 400 \mathrm{~Hz})$. Differenzen zwischen linearer lokaler Theorie 

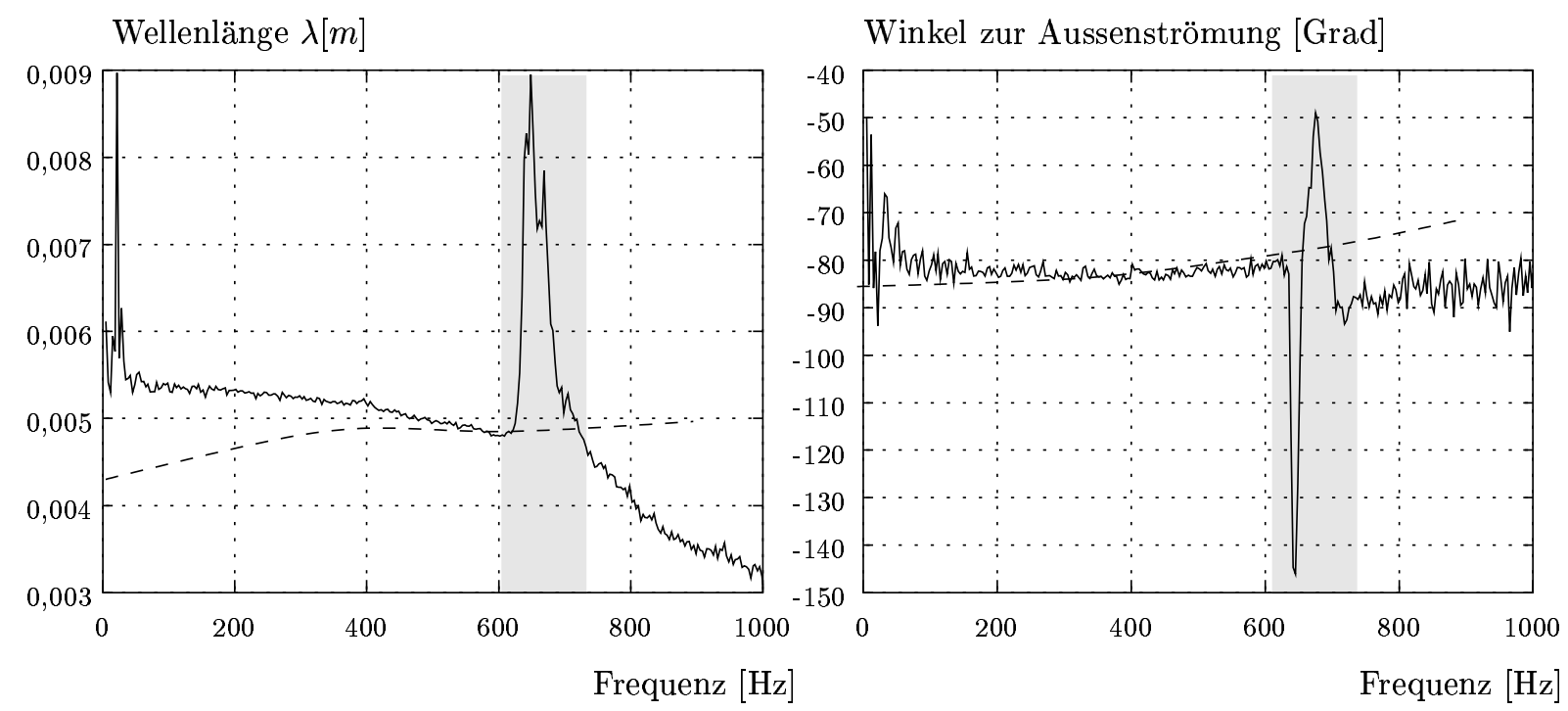

Abbildung 4.14: Gemessene Wellenlänge und Ausbreitungsrichtung der instationärer Instabilitäten im Vergleich mit Ergebnissen linearer Stabilitätsanalysen. $\left(x / c=0,3, U_{\infty}=28 \mathrm{~m} / \mathrm{s}\right)$ (gestrichelte Linien)

und Experiment findet man vor allem in den Wellenlängen laufender Instabilitätswellen für kleine $(f<100 \mathrm{~Hz})$ und große $(f>700 \mathrm{~Hz})$ Frequenzen. In diesem Bereich ist von einem schlechten Nutzsignal/Störsignal-Verhältnis auszugehen, da Instabilitäten wesentlich schwächer angefacht sind. Resultat sind systematische Beeinflussung der bestimmten Wellenlängen zu höheren bzw tieferen Werten.

Leider befand sich im Bereich $(600 \mathrm{~Hz}<f<750 \mathrm{~Hz})$ eine Resonanz der Hitzdrahtsonde ${ }^{6}$. Dieser Bereich wurde in der Abbildung 4.14 schraffiert dargestellt.

Nachdem nun eine hinreichende Übereinstimmung zwischen den Vorhersagen der linearen lokalen Theorie bzgl. der am meisten angefachten Wellenlängen und Ausbreitungsrichtungen und den im Experiment ermittelten Parametern instationärer Störungen festgestellt wurde, kann man nun die Vorhersagen der linearen Theorien bzgl. der Störungsentwicklung mit den Ergebnissen aus dem Experiment vergleichen.

\subsubsection{Stromabentwicklung instationärer Störungen}

Die Lage der neutralen Linie wurde durch numerische Instabilitätsanalysen bei $x / c=0,038$ fuer eine Frequenz von $f=500 \mathrm{~Hz}$ und eine spannweitige Wellenlänge von $\lambda_{y_{c}}=4 \mathrm{~mm}$ detektiert. Die Rechnungen wurden von Hein [22] mit dem Programm „NOLOT/PSE“

\footnotetext{
${ }^{6}$ Man kann der Abbildung entnehmen, daß es sich um eine Resonanz zur Biegeschwingung des Sondenkörpers handelt, der beide Hitzdrähte auch orthogonal zur Profiloberfläche bewegt und somit das SNR sehr ungünstig beeinflußt.
} 


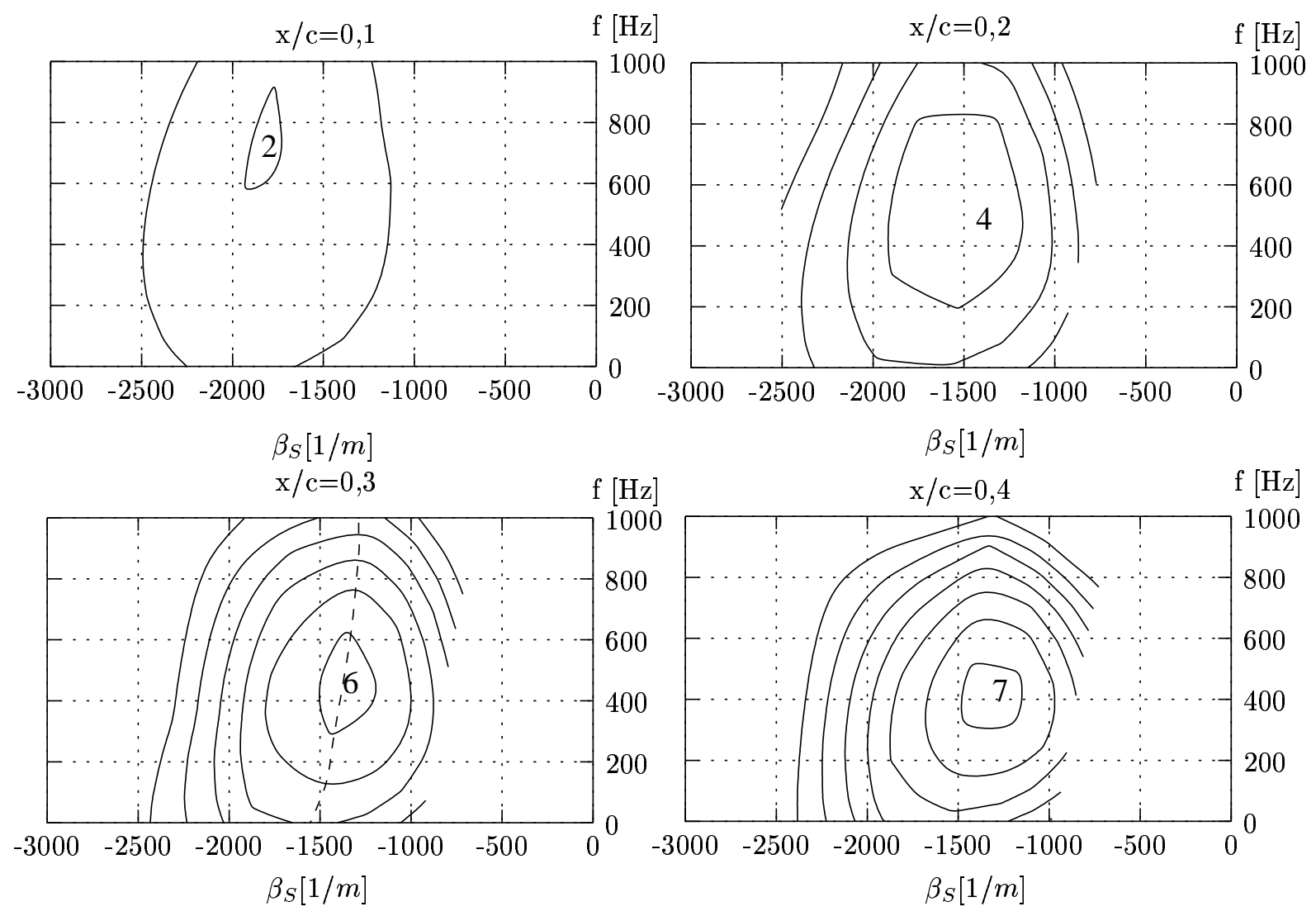

Abbildung 4.15: Isolinien konstanter relativer Störamplitude $\ln \left(u_{s} / u_{0}\right)$ in Abhängigkeit von Wellenzahl, Profiltiefe und Frequenz. Gestrichelt: Wellenzahl mit größter integraler Anfachung zu vorgegebener Frequenz. Ergebnisse numerischer Instabilitätsanalysen nach linearer lokaler Theorie von Hein [22]. $U_{\infty}=28 \mathrm{~m} / \mathrm{s}$ 

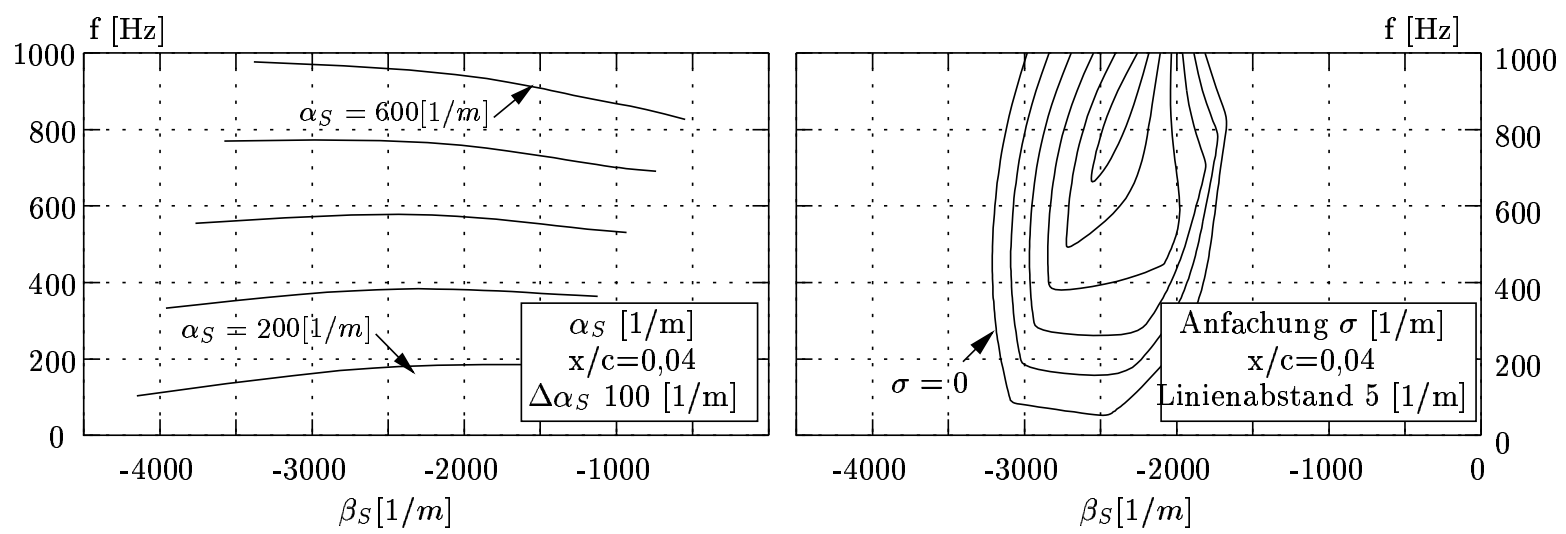

Abbildung 4.16: Isolinien konstanter Anfachung in der Nähe der neutralen Linie entsprechend lokaler linearer Theorie

\begin{tabular}{|c|c|c|c|c|c|c|c|}
\hline$x / c$ & $\alpha_{S}[1 / \mathrm{m}]$ & $\beta_{S}[1 / m]$ & $\mathrm{f}[\mathrm{Hz}]$ & $\gamma\left[^{\circ}\right]$ & $\lambda[\mathrm{mm}]$ & $\lambda / \delta_{99,9}[1]$ & $\lambda / \delta_{1}[1]$ \\
\hline \hline 0,1 & 350 & -1700 & 580 & $-78,4$ & 3,62 & 3,6 & 13,4 \\
\hline 0,2 & 230 & -1200 & 480 & $-79,1$ & 5,14 & 3,8 & 14,0 \\
\hline 0,3 & 200 & -1000 & 420 & $-78,7$ & 6,16 & 4,1 & 15,1 \\
\hline
\end{tabular}

Tabelle 4.1: Parameter der am meisten angefachten Wellen gemäß linearer lokaler Theorie

[57] unter der Verwendung einer linearen Theorie durchgeführt.

Die Abbildung 4.16 zeigt Isolinien der Anfachung $\sigma$ in Abhängigkeit von der Querwellenzahl $\beta$ und der Frequenz $f$ für eine feste Profiltiefenposition $x / c=0,04$ in der Nähe der neutralen Linie. Alle Störungen mit Parameterkombinationen innerhalb einer der dargestellten Isolinien werden durch die Querströmungsinstabilität angefacht. Diese Störungen liegen im Wellenzahlenbereich von $1100 / m$ bis 1900/m, d.h. sie besitzen Querwellenlängen von 3,3 bis 5,7mm (Querwellenzahl und Wellenlänge korrespondieren über die Beziehung $\beta=2 \pi / \lambda)$. Die zugehörigen Frequenzen liegen im Bereich 50bis1000Hz.

In der Abbildung 4.17 ist die Entwicklung der am meisten angefachten Wellenzahlen für verschiedene Profiltiefen dargestellt. Man erkennt ein relativ breites Gebiet $(\alpha, \beta, f)$ mit nicht negativen Anfachungswerten. Die Maxima dieses Anfachungsgebietes sind in der Tabelle 4.1 zusammengefaßt.

Für größerwerdende Profiltiefen verlagert sich die am meisten angefachte Instabilitätswelle von kleineren zu größeren Wellenlängen. Ihre Frequenz wird mit der Profiltiefe kleiner. Ebenfalls sinkt der Wert ihrer Anfachung. Die Gesamtverstärkung von initialen Störungen ergibt sich aus dem Integral der Anfachung für einen konstanten Frequenzwert. Die maximale integrale Anfachung wird für diese Querwellenzahl im Bereich um 400Hz von 

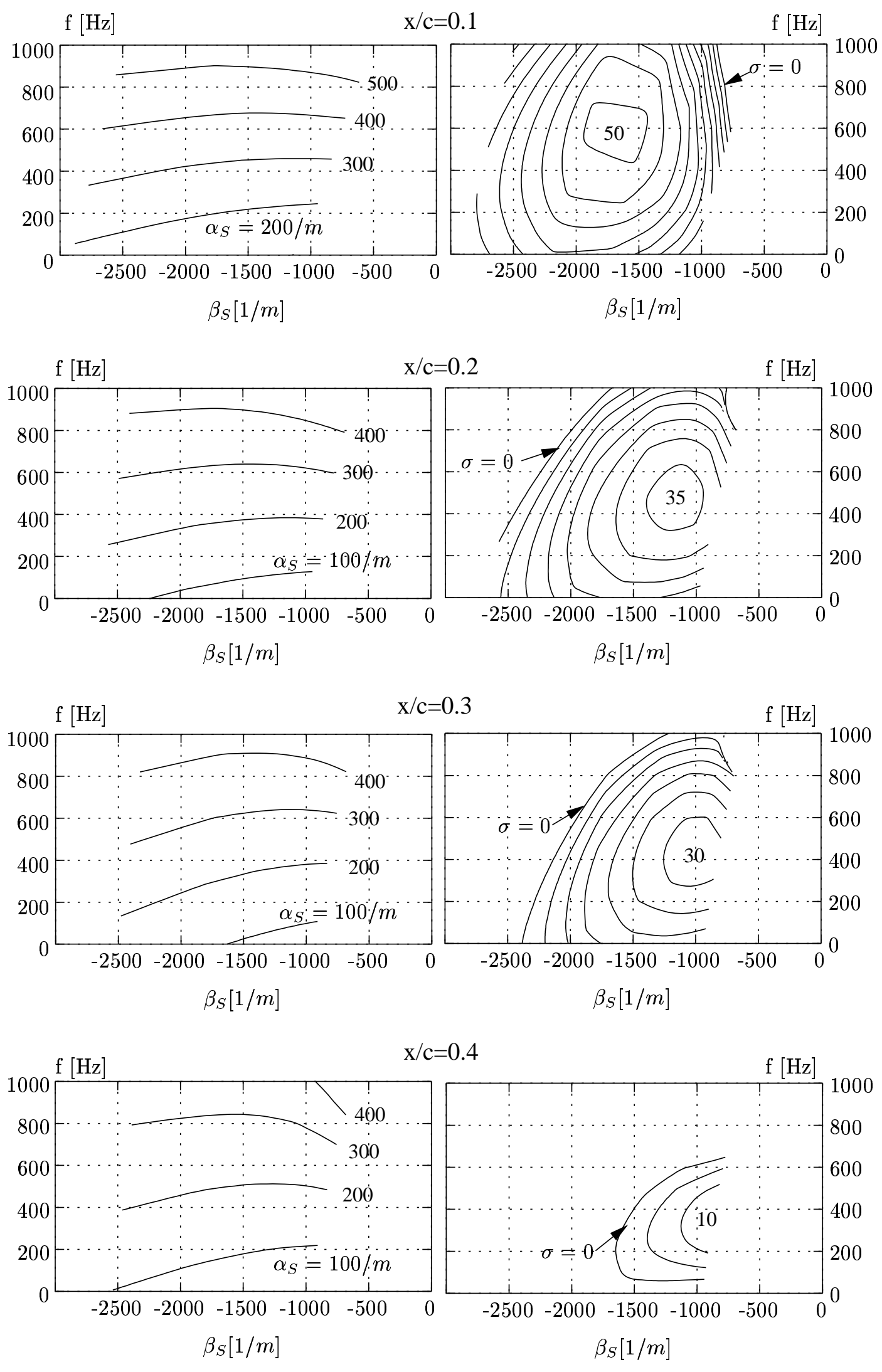

Abbildung 4.17: Isolinien konstanter Anfachung in Abhängigkeit von Wellenzahl, Profiltiefe und Frequenz. Ergebnisse numerischer Instabilitätsanalysen nach linearer lokaler Theorie von Hein [22]. $U_{\infty}=28 \mathrm{~m} / \mathrm{s}$ 


\begin{tabular}{|c|c|c|c|c|c|c|c|}
\hline$x / c$ & $\alpha_{S}[1 / \mathrm{m}]$ & $\beta_{S}[1 / m]$ & $\mathrm{f}[\mathrm{Hz}]$ & $\gamma\left[^{\circ}\right]$ & $\lambda[\mathrm{mm}]$ & $\lambda / \delta_{99,9}[1]$ & $\lambda / \delta_{1}[1]$ \\
\hline \hline 0,1 & 450 & -1800 & 700 & $-76,0$ & 3,38 & 3,4 & 12,5 \\
\hline 0,2 & 250 & -1500 & 500 & $-80,5$ & 4,13 & 3,1 & 11,2 \\
\hline 0,3 & 220 & -1350 & 430 & $-80,7$ & 4,59 & 3,1 & 11,2 \\
\hline 0,4 & 180 & -1300 & 400 & $-82,1$ & 4,79 & 2,5 & 8,6 \\
\hline
\end{tabular}

Tabelle 4.2: Parameter der Wellen mit der größten integralen Anfachung gemäß linearer lokaler Theorie

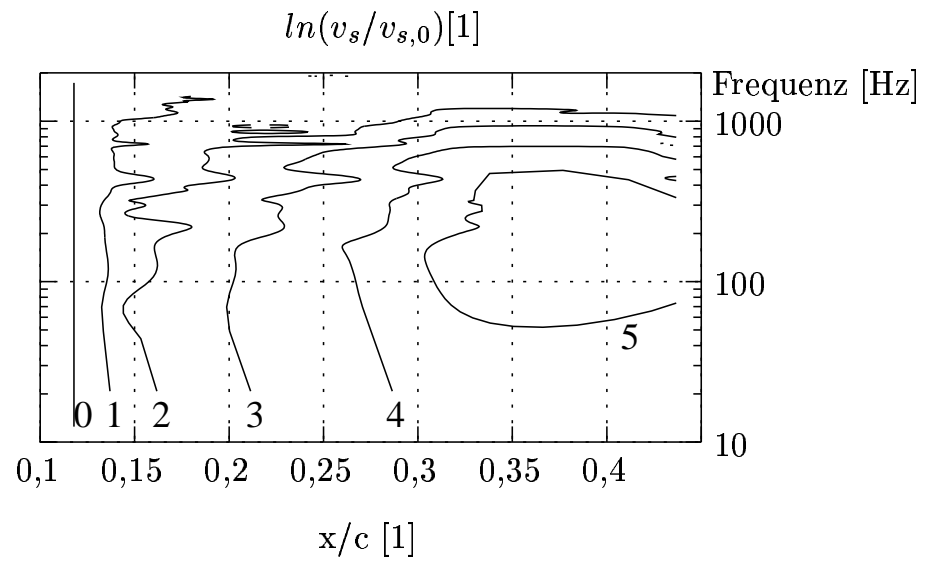

Abbildung 4.18: Experimentell ermittelte Entwicklung der relativen $v_{s}$-Amplitude über der Profiltiefenposition und der Frequenz. $U_{\infty}=28 \mathrm{~m} / \mathrm{s}$

der linearen lokalen Theorie vorhergesagt (vgl. Abbildung 4.15), und das steht in guter Übereinstimmung mit den experimentellen Ergebnissen, dargestellt in Abbildung 4.18.

Da die Abbildung 4.18 sich schlecht für einen Vergleich mit den numerischen Werten eignet, wurde in Abbildung 4.19 die Störungsentwicklung für zwei Frequenzen ( $\mathrm{f}=400 \mathrm{~Hz}$ und $\mathrm{f}=700 \mathrm{~Hz}$ ) ausgewählt und mit den Ergebnissen der integralen Anfachung nach linearen Theorien mit und ohne Parallelströmungsannahme sowie mit und ohne Krümmungstermen verglichen. Zunächst wird die Entwicklung der $400 \mathrm{~Hz}$ - Komponente diskutiert.

Analog zu den Ergebnissen für die stationären Störungen führt auch bei den instationären Störungen die Berücksichtigung der Grenzschichtänderung durch Verzicht auf die Parallelströmungsannahme auf ein schnelleres vorhergesagtes Störungswachstum. Auch die Auswirkungen der Krümmungsterme auf die Entwicklung instationärer Störungen ähneln den Auswirkungen auf stationäre Störungen. Die Oberflächenkrümmung läßt die instationären Störungen langsamer wachsen. Sie wirkt stabilisierend.

Im Unterschied zu den Ergebnissen der stationären Störungen wird die experimen- 

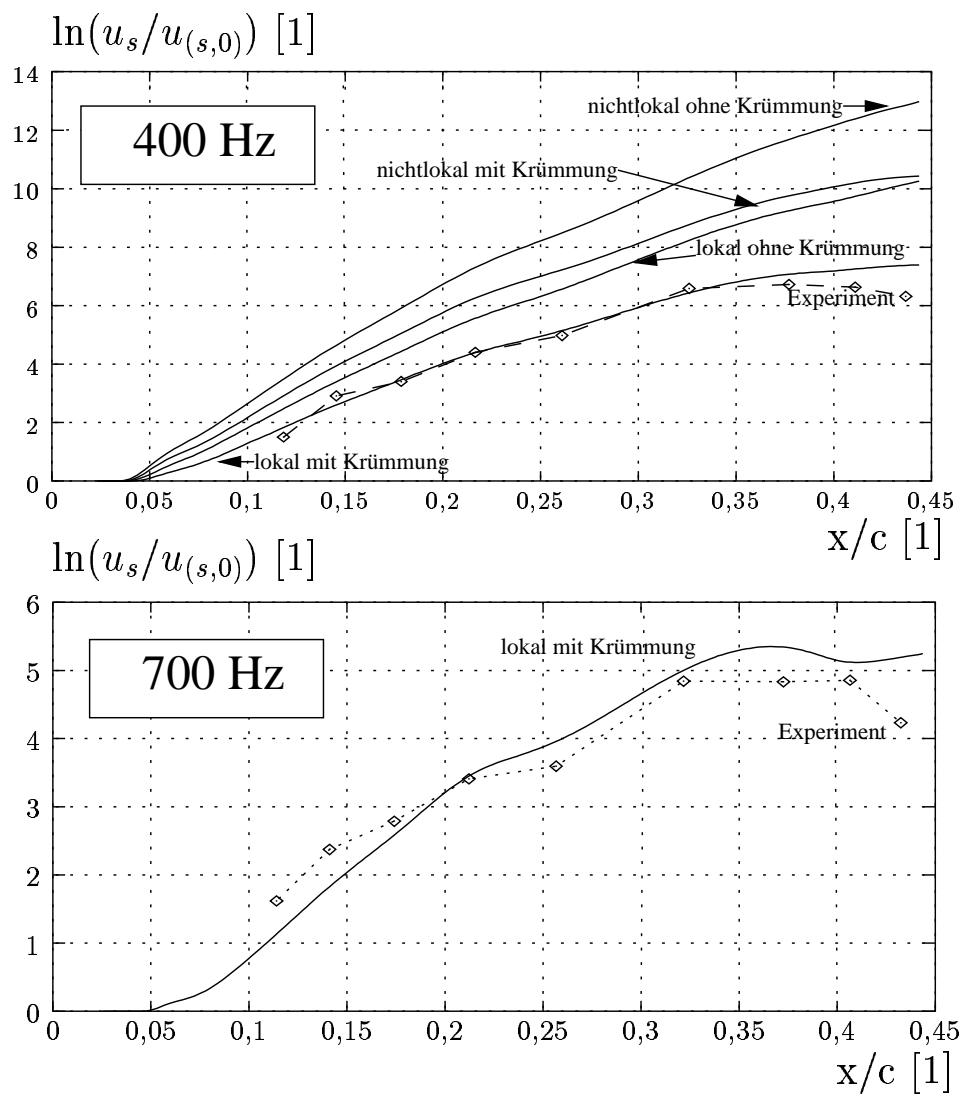

Abbildung 4.19: Vergleich von numerischer und experimentell ermittelter relativer Störungsamplitudenentwicklung $u_{s} / u_{(s, 0)}$ für Störkomponenten der Querwellenzahl $\beta=$ $1049 / m$ und den Frequenzen $f=400 H z, f=700 \mathrm{~Hz}$. 
tell bestimmte Störungsentwicklung nur unzureichend von den mathematisch konsistenten Theorien wiedergegeben. Sowohl die nichtlokale Theorie mit Berücksichtigung der Oberflächenkrümmung als auch die lokale Theorie ohne Krümmung überschätzen das Störungswachstum. Eine sehr gute Übereinstimmung zeigen die Ergebnisse für die lokale Theorie mit Krümmungstermen. Die Ursache für das Scheitern der nichtlokalen Theorien kann einerseits eine Überschätzung der destabilisierenden Wirkung der Nichtlokalität der Grenzschicht sein, da alle Ergebnisse mit Berücksichtigung der Grenzschichtentwicklung ein weit schnelleres Störungswachstum vorhersagen als gemessen wurde. Allerdings wächst die Grenzschicht im betrachteten Gebiet $0,12 \leq x_{c} / c \leq 0,33$ nur sehr langsam und die Stromlinienkrümmung ist schwach. Somit werden diese Voraussetzungen der nichtlokalen Theorien gut erfüllt und diese mögliche Ursache scheint zumindest unwahrscheinlich. Eine weitere mögliche Ursache können frühe nichtlineare Effekte sein, die ja in der linearen Theorie vernachlässigt werden. Infrage kommen hier einerseits Wechselwirkungen zwischen stationären Querströmungswirbeln und instationären Störungen (die, wie das nächste Kapitel zeigen wird, schon früzeitig beginnen), andererseits auch Wechselwirkungen zwischen den instationären Störungen untereinander. Die Veränderung der zeitlich und räumlich gemittelten Grundströmung scheidet als Ursache aus, da sie sich erst bei größeren Profiltiefen entwickelt, wie im nächsten Kapitel gezeigt wird. Endgültig Klarheit kann hier nur eine Stabilitätsanalyse unter Verwendung einer nichtlinearen Theorie bringen.

Für den Vergleich der $700 \mathrm{~Hz}$ - Komponente steht leider nur die lineare lokale Theorie mit Berücksichtigung der Krümmungsterme zur Verfügung. Auch hier zeigt sich eine gute Übereinstimmung im Bereich $0,175 \leq x_{c} / c \leq 0,32$. Für Positionen stromauf von $x_{c} / c=$ 0,175 sind die Amplituden der 700Hz - Komponente jedoch so klein, daß systematische Fehler z.B. durch Sondenschwingungen die experimentell ermittelten Amplituden nach oben verfälschen.

Der Bereich großer Amplituden $\left(x_{c} / c>0,33\right)$ wird von der verwendeten linearen Theorie nicht korrekt wiedergegeben, da durch die großen Störungsamplituden die nichtlinearen Glieder an Einfluß gewinnen und damit nicht mehr vernachlässigt werden dürfen. Resultierend wird das Störungswachstum primärer laufender Instabilitäten überschätzt. Das folgende Kapitel widmet sich diesem Bereich nicht mehr zu vernachlässigender nichtlinearer Glieder. 


\section{Kapitel 5}

\section{Nichtlineare Entwicklung der Grenzschicht}

Der linearen Entwicklung von Störungen innerhalb der Grenzschicht schließt sich die nichtlineare Entwicklung an, sobald die Störungsamplituden eine bestimmte Größe erreichen. Der Begriff „lineare Entwicklung“ entstammt der Stabilitätstheorie, die bereits auf der Ebene der Navier - Stokes - Gleichungen linearisiert (siehe Anhang A auf 97), und bezeichnet den Bereich der Strömung, der unter dieser Näherung beschreibbar bleibt.

In den aus den Navier - Stokes Gleichungen durch Subtraktion der Gleichungen der zeitlich gemittelten Größen gewonnenen Störungsdifferentialgleichungen

$$
\begin{aligned}
\nabla \vec{q} & =0 \\
\partial \vec{q} / \partial t-1 / \operatorname{Re} \nabla^{2} \vec{q}+(\vec{q} \nabla) \vec{U}+(\vec{U} \nabla) \vec{q}+\nabla p & =(\vec{q} \nabla) \vec{q}
\end{aligned}
$$

generiert der Term

$$
(\vec{q} \nabla) \vec{q}
$$

aus harmonischen Störungen der Form

$$
\overrightarrow{q_{1}}=A_{1} \exp \left(i\left(\gamma_{1} \vec{k}+\omega_{1} t\right)+\right.\text { c.c. }
$$

harmonische Störungen $\omega_{n}=n \omega_{1}$ und $\gamma_{n}=n \gamma_{1}$ mit $\mathrm{n}=2,3, \ldots$ (nichtlinear generierte Höherharmonische) sowie $\mathrm{n}=0$ (Grundströmungsverformung). Zusätzlich werden aus Überlagerungen zweier Moden $\left(n_{1} \omega, m_{1} \vec{k}\right)$ und $\left(n_{2} \omega, m_{2} \vec{k}\right)$ Moden mit $n_{1} \pm n_{2}$ und $m_{1} \pm m_{2}$ generiert. Diese Entwicklung ist für kleine Amplituden A vernachlässigbar, da jede dieser so entstehenden Moden eine Amplitude der Größenordnung $A^{2}$ (oder höher) hat. Sind jedoch Störungen mit großer Amplitude beteiligt, sei es durch eine bis dato lineare Anfachung oder/und durch hohe Anfangsamplituden, so ist die Näherung der linearen Theorie, den nichtlinearen Term zu vernachlässigen, nicht mehr berechtigt: Einerseits greift die Grundströmungsverformung direkt in die Stabilitätseigenschaften der Grenzschicht ein, andererseits beginnt ein Störungsenergietransport hin zu den nichtlinear generierten Höherharmonischen. Ersteres führt zu einer qualitativen Veränderung des Störungswachstums durch 
eine eventuelle Verschiebung der am meisten angefachten Störungen zu anderen Frequenzen und Wellenzahlen. Der Störungsenergietransport verringert das durch die lineare Stabilitätstheorie vorhergesagte Störungswachstum. Diese Veränderungen manifestieren sich in dem Phänomen der „Sättigung“: Linear angefachte Moden bleiben hinter den vorhergesagten Anfachungsraten zurück oder stagnieren sogar.

Diese Beschränkung der linearen Theorie hebt das Nichtlineare PSE - Verfahren auf, indem es simultan ein gekoppeltes System von PSE für die relevanten Moden ${ }^{1}$ löst. Die Durchführung einer Nichtlinearen PSE ist jedoch nicht leicht zu automatisieren, da man sich zunächst erst einmal einen Überblick darüber verschaffen muß, welches die relevanten Moden für das konkrete Problem sind. Dazu sind detailierte Kenntnisse der Störungsanfangs- und Randbedingungen sowie der linearen Stabilitätseigenschaften des Untersuchungsgegenstandes von Nöten.

Das große Interesse, das man gegenwärtig diesem Bereich der Störungsentwicklung entgegenbringt, entspringt dem Wunsch, mit den vergleichsweise billigen Mitteln der Stabilitätsanalyse die Grenzschichtentwicklung soweit wie möglich beschreiben zu können. Das Fernziel ist die Vorhersage des Umschlagsortes, wozu die genaue Kenntnis der Ausgangsituation des laminar - turbulenten Umschlags gehört. Der Umschlag selbst wird mutmaßlich allerdings nicht mit den Mitteln der Stabilitätsanalyse beschreibbar sein, da sie nur Amplitudenentwicklungen von Störungen in einer laminaren Grenzschicht angibt.

Die Aufgabe des Experiments liegt in diesem Zusammenhang in der Schaffung einer experimentellen Basis für das späte Stadium der Grenzschichtentwicklung, um spätere nichtlineare Rechnungen an ihm validieren zu können. Darüber hinaus kann man die beobachteten nichtlinearen Effekte beschreiben und Vergleiche mit anderen Experimenten ähnlicher Thematik durchführen, um beispielsweise den Krümmungseinfluß auf das späte Stadium der laminaren Grenzschichtentwicklung einschätzen zu können.

\subsection{Die Grundströmungsverformung}

Der nichtlineare Term in Gleichung 5.3 generiert, neben den Höherharmonischen einer vorgegebenen Mode und den durch Wechselwirkungen mit anderen Moden entstehenden Summen-und Differenzmoden, auch eine sogenannte $(\mathbf{0 , 0 )}$-Mode. Unter diesem Begriff wird die gesamte durch den nichtlinearen Term verursachte Änderung der zeitlich und räumlich gemittelten Grenzschichtgeschwindigkeiten subsumiert. Die (0,0)-Mode stellt daher eine Größe dar, anhand derer man den Gültigkeitsbereich der linearen Theorie abschätzen kann, während die nichtlinear generierten Störungen höherer Ordnung aus dem breiten Band der angefachten Störungen nicht ohne weiteres identifiziert werden können. Ihr Vorhandensein ist ein relativ leicht $\mathrm{zu}$ detektierendes, hinreichendes Kriterium einer nichtlinearen Entwicklung der Grenzschicht:

\footnotetext{
${ }^{1}$ Die Beschränkung auf die relevanten Moden ist wichtig, da Rechenaufwand und Speicherbedarf für das Lösen des gekoppelten Systems von PSE mit der berücksichtigten Anzahl von Moden schnell anwachsen.
} 
Mit der Bezeichnung

$$
\bar{q}:=\frac{1}{T} \int_{0}^{T} q\left(x_{0}, y_{0}, z_{0}, t\right) d t
$$

für eine zeitliche Mittelung und

$$
<\bar{q}>:=\frac{1}{Y} \int_{0}^{Y} \bar{q}\left(x_{0}, y, z_{0}\right) d y
$$

für die räumlich, spannweitige Mittelung kann man diese Störung kurz mit

$$
\vec{U}_{(0,0)}(x, z):=<\vec{U}_{N L}>-<\vec{U}_{L}>
$$

bezeichnen, wenn der Index L die nach linearer Theorie ermittelte Grenzschichtströmung bezeichnet und NL der Index der nichtlinearen Theorie ist.

Leider existieren derzeit keine nichtlinearen Rechnungen zum vorliegenden Experiment. Aus diesem Grund nähere ich die Ergebnisse der nichtlinearen Theorie durch meine Messungen, ein Schritt, der normalerweise in umgekehrter Richtung getan wird, ohne ihn breiter $\mathrm{zu}$ würdigen.

Allerdings ist es nicht unproblematisch, die Differenz von experimentell ermittelten, räumlich und zeitlich gemittelten und numerisch ermittelten Grenzschichtgeschwindigkeitsprofilen als Haupströmungsstörung darzustellen, da noch zahlreiche andere Fehler Ursachen von Unterschieden sein können. Diese Fehler wurden bereits im Kapitel "Grundströmung“ diskutiert. Dieses Verfahren ist meiner Meinung nach trotzdem geeignet, einen Eindruck vom Einsetzen nichtlinearer Prozesse zu geben.

Die Darstellungen in der Abbildung 5.1 zeigen für die Profiltiefe von $\mathrm{x} / \mathrm{c}=0,115 \mathrm{im}$ wesentlichen den großen relativen Fehler bei der Messung des Geschwindigkeitsprofils. Die Grenzschicht ist an dieser Stelle noch zu dünn, die Geschwindigkeitsgradienten $\partial U_{s} / \partial z$ zu groß, um die erforderliche relative Genauigkeit zu erzielen. Ähnliches gilt bei den anderen dargestellten Profiltiefenpositionen für die Bereiche $z / \delta_{99,9}<0,2$, die in der Abbildung schraffiert unterlegt sind. In den verbleibenden Gebieten erkennt man beim Übergang von $x / c=3,10 \mathrm{zu} x / c=4,25$ die sich ausbildende Hauptströmungsstörung. Sie bewirkt eine Überhöhung des Geschwindigkeitsprofils für wandnahe Positionen sowie eine Absenkung für größere $z / \delta_{99,9^{-}}$Werte. Diese Gestalt der $(\mathbf{0 , 0})$-Mode ist charakteristisch für Strömungen unter dem Regime der Querströmungsinstabilität mit gut ausgeprägten stationären Wirbeln. Ihre Amplitude ist aber mutmaßlich abhängig von den Charakteristiken der Anfangsstörungen am Beginn ihrer Entwicklung und deren Stromab-Evolution.

Der Beginn der nichtlinearen Entwicklung ist somit auf den Bereich $x_{c} / c<0,425$ eingeschränkt. 

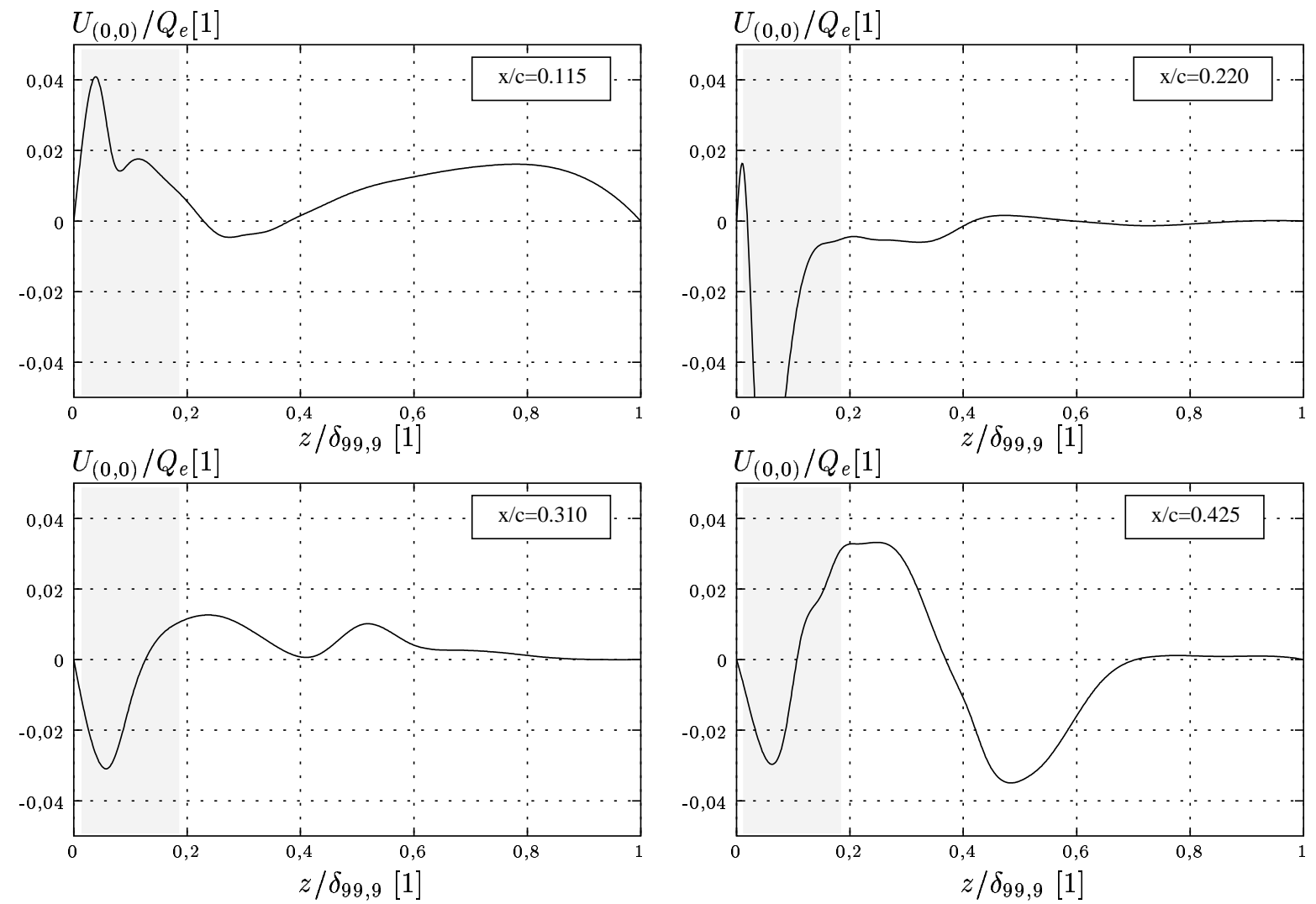

Abbildung 5.1: Entwicklung der $U_{s}$-Hauptströmungsstörung für die Profiltiefen $x / c=$ $0,115,0,220,0,310,0,425$ 


\subsection{Wechselwirkungen stationärer und instationärer Störungen}

Wie bereits in der Diskussion der möglichen Ursachen zur mangelhaften Vorhersage der Amplitudenentwicklung instationärer Störungen erwähnt, werden Wechselwirkungen verschiedener Instabilitätsmoden untereinander in der linearen Theorie vernachlässigt. Die lineare Theorie beschreibt also nur so lange die Physik der Grenzschichtentwicklung korrekt, wie diese Wechselwirkungen gegen die primär betrachteten Störungen vernachlässigbar bleiben. Da Wechselwirkungen laufender Moden untereinander in diesem Experiment nicht untersucht werden können, beschränke ich mich im folgenden auf die Diskussion der Wechselwirkung laufender Störungen mit den stationären Querströmungswirbeln.

Betrachtet man eine instationäre laufende Mode in der Grenzschicht ohne Wechselwirkung mit stationären Moden, so würde sich eine dem Betrag ihrer Amplitudenfunktion proportionale RMS-Wert-Verteilung ergeben. Da die Amplitudenfunktion in der linearen Theorie nicht von der Spannweite abhängig ist, erwartet man also eine in $z$ - Richtung veränderliche, aber in spannweitige Richtung konstante RMS-Wert-Verteilung der untersuchten Geschwindigkeitskomponente (vergl. Normalmodenansatz in (4.4) auf Seite 34).

Wie jedoch Abbildung 5.2 für $R M S\left(v_{s}\right) / Q_{e}$ illustriert, sind bereits in einer frühen Phase der Störungsentwicklung $\left(x_{c} / c=0,145\right)$ Wechselwirkungen zwischen stationären und instationären Störungen zu beobachten. Deutlich zeigt sich eine spannweitige Variation des RMS-Wertes mit einer mit den stationären Wirbeln übereinstimmenden Wellenlänge von $\lambda=6 \mathrm{~mm}$. Das Maximum der Variation befindet sich bei allen untersuchten Profiltiefen im Bereich $z / \delta_{99,9}=0,3$ bis 0,4. Die Amplitude der Variation (halber Maximum-Minimum Abstand) liegt bei ca. $25 \%$ des spannweitigen Mittelwertes und ist damit an keiner der dargestellten Profiltiefen vernachlässigbar gegen den spannweitigen Mittelwert $<R M S\left(v_{s}\right)>$. Analoge Beobachtungen machte bereits Lerche [14]. Interpretiert man ihm folgend diese Wechselwirkungen im Bild von Instabilitätswellen, so erzeugt eine primär angefachte Instabilitätswelle durch ihre Wechselwirkung mit den stationären Querströmungswirbeln eine in Frequenz und Stromabwellenzahl identische, aber in der Querwellenzahl entgegengesetzte Welle, deren Amplitude man aus der Modulation des RMS-Wertes bestimmen kann. Wie die Stabilitätsanalysen zeigen ist diese Welle primär gedämpft.

Welchen Einfluß diese frühen Nichtlinearitäten auf die Störungsentwicklung primär angefachter Störungen haben, können erst Rechnungen auf der Basis nichtlinearer Theorien zeigen. Mutmaßlich wirkt deren Einsetzen allerdings dämpfend und ist die Ursache für das hinter den Erwartungen linearer Theorien zurückbleibende Wachstum instationärer Störungen, wie es im vorigen Kapitel diskutiert wurde.

Der Beginn der nichtlinearen Entwicklung ist somit bereits auf den Bereich $x_{c} / c<0,145$ eingeschränkt. 

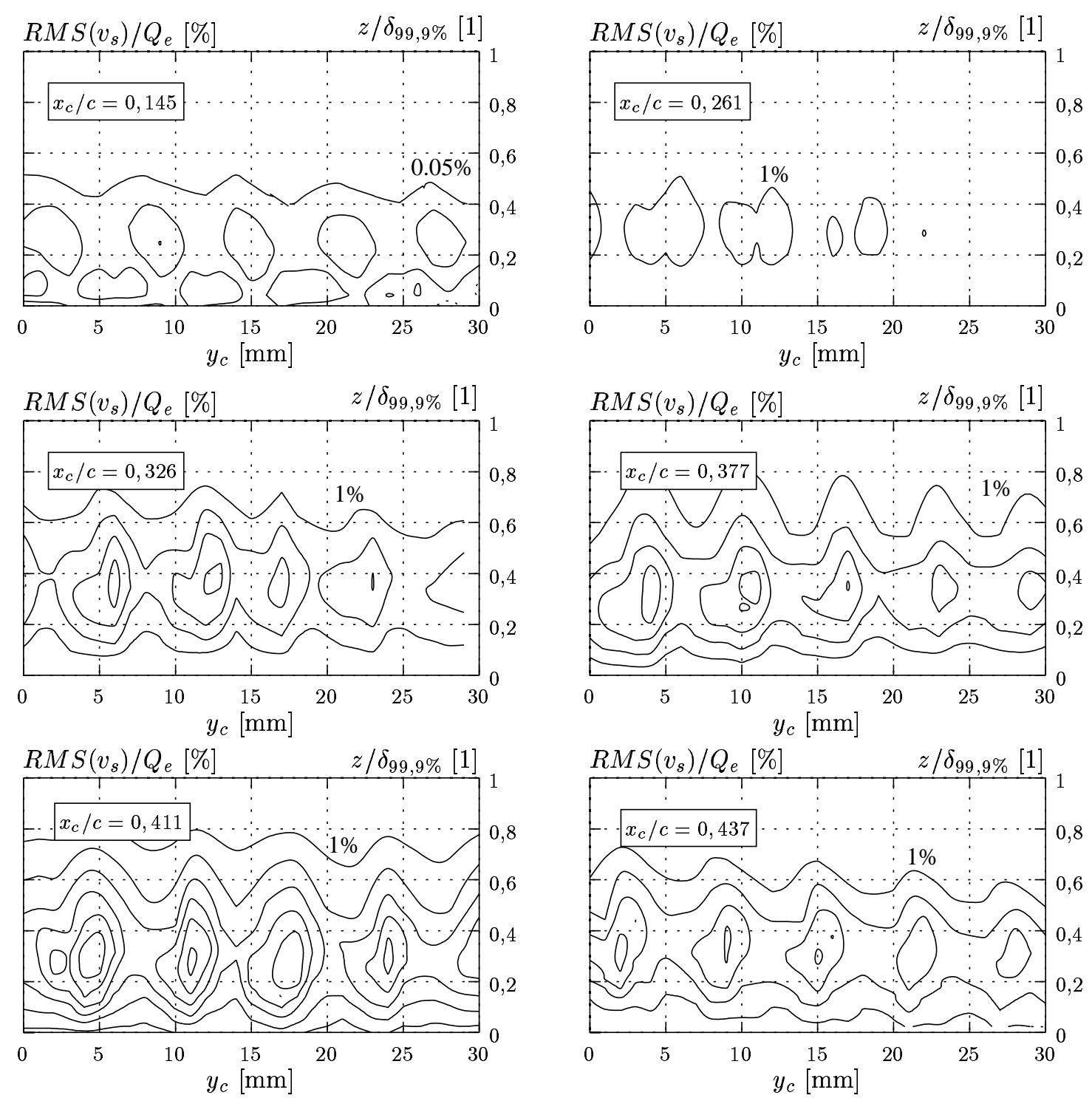

Abbildung 5.2: Isolinien der normierten Querströmungs-RMS-Werte für verschiedene Profiltiefenpositionen $x / c=0,1455,0,261,0,326,0,377,0,411,0,437$ 


\begin{tabular}{|c|c|c|}
\hline Sättigungsamplituden & max. stat. Amplitude & max. RMS-Wert \\
\hline \hline DLR Prinzipexp. Schiebende Platte & $20 \%$ & $3 \%$ \\
\hline HQ26, schiebend & $7 \%(10 \%)$ & $7 \%(20 \%)$ \\
\hline
\end{tabular}

Tabelle 5.1: Vergleich der gemessenen Sättigungsamplituden zwischen dem DLR Prinzipexperiment „Schiebende Platte“ und dem konvex gekrümmten Tragflügelprofil HQ26. Alle Angaben in Prozent der Außenströmungsgschwindigkeit. Die Werte in Klammern wurden unter Verwendung von periodischen Rauhigkeitselementen gemessen.

\section{$5.3 \quad$ Sättigung}

Sättigung entstammt, ähnlich wie der Begriff (0,0)-Mode, ebenfalls der Stabilitätstheorie. Damit wird das Phänomen der Stagnation des Störungswachstums trotz linearer Anfachung umschrieben, das durch Vernachlässigung des nichtlinearen Terms in Gleichung 5.3 nicht mit dieser Theorie erfaßt werden kann. In ihn fließen alle Ursachen ein, die zum letztendlichen Stagnieren der Störamplitudenentwicklung führen: Nicht nur die Störungsenergieverluste durch Streuung in höhere Moden, sondern auch die Auswirkungen der Hauptströmungsdeformation auf die Stabilitätseigenschaften der Grenzschicht durch die (0,0)-Mode und die Charakteristiken der Anfangsstörungen sowie deren Entwicklungsgeschichte (siehe Deyhle und Bippes in [34]).

Die Sättigungsamplitude einer Mode, dh. die letztendlich alles aufnehmende skalare Größe, vereint so in sich eine Vielzahl physikalischer Prozesse und ist damit nicht universell, sondern stark abhängig von der betrachteten Situation.

Trotz dieser gravierenden Einschränkungen ist der Begriff Sättigungsamplitude noch heute auf dem Gebiet der Transitionsvorhersage von einiger Bedeutung, um, zum Beispiel, unterschiedliche Experimente unter ähnlichen Bedingungen zu vergleichen. Darüber hinaus ist das Gebiet der Sättigung ein Prüfstein von modernen Strömungsberechnungsverfahren auf der Grundlage von Instabilitätsanalysen.

In der Tabelle 5.1 sind experimentell ermittelte Sättigungsamplituden zweier vergleichbarer Experimente dargestellt. Als Definition der Sättigungsamplitude wurde im stationären Fall die Hälfte der maximalen spannweitigen Modulation von $U_{s}$ auf Höhe des Querströmungsmaximums gewählt. Im instationären Fall wurde der RMS-Wert der mit einer Grenzfrequenz von $2000 \mathrm{~Hz}$ tiefpassgefilterten $u_{s}$ Geschwindigkeitsfluktuation angegeben.

Verglichen wurde mit dem DLR Prinzipexperiment „Schiebende Platte“. Dabei handelt es sich um eine ebene Platte, die im Winkel von -45 Grad schiebend zur Hauptströmungsrichtung angeordnet ist und der ein Verdrängungskörper an der Oberseite einen nahezu konstanten negativen Druckgradient aufprägt. (Details siehe Deyhle [47], Lerche [14]). Beide Experimente wurden im gleichen Windkanal, dem DNW/NWB, im gleichen Re- 
Bereich durchgeführt. Damit kann man davon ausgehen, daß zumindest ähnliche äußere Bedingungen bei beiden Experimenten vorlagen. Die Sättigungsamplituden wurden mit der Grenzschichtrandgeschwindigkeit normiert.

Im vorliegenden Experiment ist die Entscheidung, ob überhaupt Sättigungsbereiche erreicht wurden, nicht endgültig zu klären, da der Druckgradient an der Profiloberseite nicht konstant und somit die Anfachung von Störungen veränderlich ist. Für das Erreichen der Sättigung spricht, daß lineare Rechnungen für große Profiltiefen deutlich höhere Störamplituden (insbesondere für instationäre Störungen) vorhersagen (vergleiche die Abbildung 4.9 auf Seite 45 zur stationären und Abbildung 4.19 auf Seite 57 zur instationären Amplitudenentwicklung). Ebenso wird das Maximum der Anfachung bereits kurz vor dem Druckminimum erreicht. Desweiteren haben Untersuchungen zum Zusammenbruch der laminaren Grenzschichtströmung, das heißt bei höheren Re-Zahlen, auch keine höheren stationären Störungsamplituden erbracht. Im Falle des RMS-Wertes wurden je nach durchlaufenem Transitionsszenario jedoch auch höhere RMS-Werte gemessen, allerdings unter den veränderten Bedingungen einer höheren Anströmungsgeschwindigkeit.

Der Vergleich beider Experimente offenbart den wesentlichen Unterschied, daß der RMS-Wert im Falle des konvex gekrümmten Profils ähnlich große Amplituden erreicht, wie die stationären Moden. Im Fall des DLR Prinzipexperiments war das nicht so. Dort erreichten die stationären Moden deutlich höhere Werte, und es wurden nur sehr kleine RMSWerte gefunden $^{2}$. Beide Experimente unterscheiden sich, neben der Oberflächenkrümmung und dem Druckgradient, auch noch in der mittleren Rauhtiefe im Vorderkantenbereich. Den $R_{z}=2,5 \ldots 3,0 \mu m$ am HQ26 stehen nur $1 \mu m$ beim Prinzipexperiment gegenüber. Dieser Wert läßt auf eine geringe Anfangsamplitude stationärer Störungen im Prinzipexperiment schließen und kann so nicht die beobachteten Sättigungsamplituden erklären. Darüber hinaus besitzen beide Experimente eine ähnliches Anfachungsverhältnis von stationären und instationären Moden: Die instationären Moden sind etwas stärker angefacht als die stationären. Demnach sind die Ursachen der unterschiedlichen Sättigungsamplituden mutmaßlich in der unterschiedlichen Oberflächenkrümmung zu suchen.

Bemerkenswert ist die Koppelung der erreichten RMS-Werte an die Stimulierung der stationären Mode (siehe Werte in den Klammern in Tabelle 5.1). Näheres über die damit zusammenhängenden Prozesse in der Grenzschicht können aber erst nichtlineare Rechnungen zeigen, die für unterschiedliche Anfangsamplitudenverteilungen zwischen stationären und instationären Moden die resultierende Entwicklung berechnen.

Der Vergleich der Ergebnisse beider Experimente macht macht aber deutlich, daß Umschlagskriterien den Einfluß der Krümmung nicht vernachlässigen dürfen, wie dies bisher bei der Anwendung des $e^{N}$-Kriteriums, des derzeit anerkanntesten Kriteriums, der Fall ist.

\footnotetext{
${ }^{2}$ Das Experiment von Lerche mit kontrollierter Anregung stationärer und instationärer Störungen ergab Sättigungsamplituden von 3\% für die stationäre Mode und bis zu 7\% für die RMS-Werte. Diese Messungen wurden allerdings im 1-m-Kanal Göttingen durchgeführt und sind damit nicht direkt vergleichbar.
} 


\section{Kapitel 6}

\section{Zusammenbruch der laminaren Grenzschichtströmung}

Irgendwann nachdem die primär vorhandenen Störungen den Sättigungszustand erreicht haben, wird die laminare Grenzschichtströmung durch eine turbulente abgelöst. Im Experiment findet man nicht mehr eine geordnete, geschichtete Grenzschichtströmung mit überlagerten stationären und instationären Störungen, sondern eine starke unregelmäßige Geschwindigkeitsschwankung um eine mittlere Geschwindigkeit. Der Übergang vollzieht sich während einer Intermittenzphase, in der sich die laminare und turbulente Strömungsform in kurzen Abständen abwechseln.

Die turbulente Strömung zeichnet sich durch eine verstärkte Energiedissipation aus. Energie aus der Fluidbewegung wird über eine Kaskade von Wirbeln von großen zu kleinen Strukturen über Reibung in Wärme umgewandelt und somit der Strömung entzogen. Damit sind auch Änderungen anderer wichtiger Eigenschaften der Grenzschicht verbunden. So wird das zeitlich gemittelte Geschwindigkeitsprofil steiler. Die Tendenz zur Strömungsablösung sinkt. Der Wärmeabtransport von den die Grenzschicht berandenden Flächen sowie die Mischungsprozesse in der Grenzschicht beschleunigt sich.

Wegen dieser qualitativen Veränderungen am Umschlagsort sind die Vorhersagen bezüglich des Umschlagsortes von großer Bedeutung für Strömungsberechnungsverfahren. Leider gestaltet sich diese Vorhersage schwierig, da die Physik des Zusammenbruchs der laminaren Strömung in der derzeitigen Form nicht von Stabilitätsanalysen erfasst wird. Aus diesem Grund greift man auf halbempirische Verfahren zurück, um die Lage des Umschlags zu beschreiben.

Halbempirische Verfahren (z.B. das $e^{N}$ - Verfahren) benutzen als Grundlage einen Katalog ähnlicher Strömungskonfigurationen, der eine Zuordnung der nach linearer Theorie ermittelten integralen Anfachung und experimentell ermittelter Umschlagsposition bietet. Diese Verfahren nehmen also den Umschlag dann an, wenn der nach linearer Theorie ermittelte N-Faktor einen bestimmten Schwellenwert erreicht. Diese Verfahren sind unzulänglich, da sie eine bereits nicht mehr die physikalische Realität beschreibende Theorie als Grundla- 
ge der Umschlagsvorhersage benutzen. Trotzdem ist ihre Bedeutung nicht zu unterschätzen, weil sie leicht durchzuführen ist und aufgrund der umfangreichen bisher existierenden Kataloge bei zweidimensionalen Grenzschichten auch schnell Anhaltspunkte zur Transitionslage liefert.

Eine Verbesserung der halbempirischen Verfahren erhofft man sich aus dem Einsatz der nichtlinearem PSE - Verfahren. Diese sollen, mit Kenntnis der konkreten Anfangsbedingungen, bereits eine physikalisch korrekte Modulation der kompletten laminaren Grenzschichtentwicklung bis in den Sättigungsbereich hinein ermöglichen. Aber auch sie beschreiben in ihrer derzeitigen Form nicht den Prozeß, der den endgültigen Zusammenbruch der laminaren Strömung einleitet.

Die nichtempirischen Verfahren, z.B. die direkte numerische Simulation (DNS), stellen Verfahren dar, die ausgehend von Anfangsstörungsamplituden die Navier-StokesGleichungen direkt zur Berechnung der Störungsentwicklung nutzen. Diese Verfahren stellen heutzutage die Krone der Strömungsberechnungsverfahren dar. Sie sind allerdings technisch nur für überschaubare Geometrien möglich und sind sowohl sehr teuer als auch aufwendig. Man setzt sie im Wesentlichen zur Validierung bei der Entwicklung halbempirischer Verfahren ein. Trotzdem werden sie in Zukunft stetig an Bedeutung gewinnen, wenngleich der numerische Windkanal noch weit ab der heutigen Möglichkeiten liegt. Die Stärke dieser Verfahren liegt vor allem in Bereichen, in denen, aufgrund sehr kleiner Amplituden, Messungen von Störungsamplituden (noch) nicht möglich sind. Um quantitative Aussagen machen zu können, müssen allerdings die Anfangsbedingungen bekannt sein. Und auch die DNS erfassen derzeit nicht die Vorgänge, die den Zusammenbruch der laminaren dreidimensionalen Strömung einleiten.

Da die physikalischen Prozesse des Umschlags weder von empirischen noch von den halbempirischen Verfahren modelliert werden, ist man bestrebt, Kriterien für einen unmittelbar bevorstehenden Umschlag zu finden, die man im Rahmen der jeweiligen angewendeten Methode detektieren kann, um mit ihnen das Kriterium des maximalen N-Faktors zu ersetzen.

\subsection{Identifikation des Umschlags}

Die Umschlagsdetektion wurde, wie in Experimenten üblich, online mit einem Oszillographen anhand des beobachteten Intermittenzfaktors der unkalibrierten Hitzdrahtsignale vorgenommen. Der Intermittenzfaktor $\gamma_{I}$ stellt dabei das zeitliche Verhältnis von turbulentem Signal zu laminaren Signal dar. Er ist somit in der voll ausgebildeten turbulenten Strömung identisch 1 und in der laminaren Strömung identisch 0. Der Übergang von einem zum anderen Zustand ist relativ scharf (vgl. Deyhle [47], Kapitel 5). Man kann nun einen beliebigen Schwellenwert als Übergang definieren. In der Literatur wählt man dafür meist den Wert $\gamma_{I}=0,5$. Die unkalibrierten Hitzdrahtsignale liefern bei kleinem lokalen Anströmungswinkel (d.h. Sondenausrichtung ungefähr in Richtung der Stromlinie am Grenzschichtrand) 


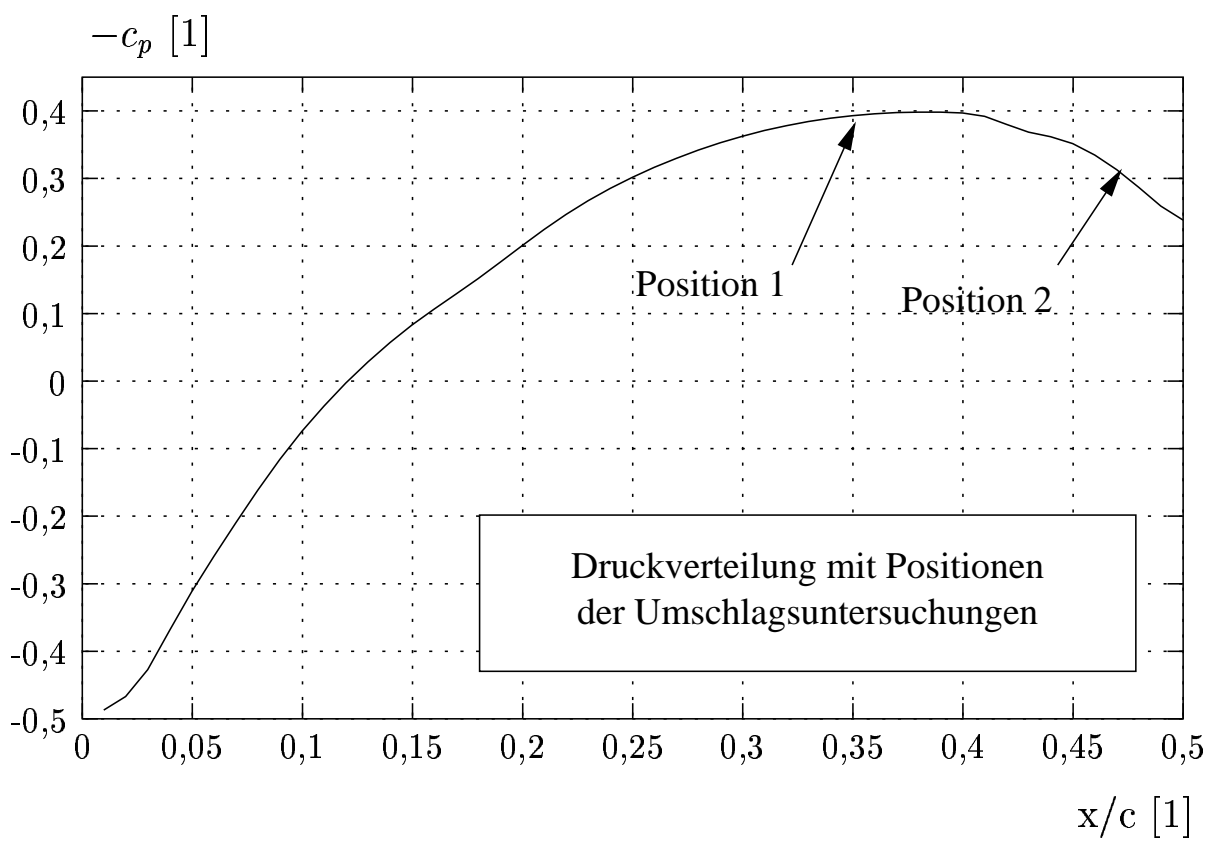

Abbildung 6.1: Lage der Profiltiefenpositionen der Umschlagsuntersuchungen bezüglich der entdimensionalisierten Druckverteilung der Profiloberseite.

ein Signal proportional zur $u_{s}$ - Geschwindigkeitskomponente der Strömung.

Zur Untersuchung des Umschlags wurde die Profiltiefenposition konstant gehalten und die Anströmungsgeschwindigkeit angepaßt. Dann wurden für die verschiedenen Anströmungsreynoldszahlen Profilreihen aufgenommen, als Basis für Offline - Untersuchungen der lokalen Strömungssituation.

\subsection{Ablauf des Umschlags}

Der Ablauf des Umschlags einer Grenzschicht ist abhängig von der bisher durchlaufenden Störungsentwicklung und der am Umschlagsort herrschenden lokalen Strömungssituation. Diesem Umstand wurde im vorliegenden Experiment Rechnung getragen. Die Untersuchungen zum Umschlag wurden an zwei Profiltiefenpositionen durchgeführt, an denen sich die Grenzschicht sowohl in den lokalen Stabilitätseigenschaften als auch in der Störungsentwicklungsgeschichte charakteristisch unterscheidet (siehe Abbildung 6.1). Im ersten Fall wird der Umschlag von der Querströmungsinstabilität dominiert. Im zweiten Fall wirkt die Tollmien-Schlichting Instabilität mit. Beide Fälle sind von praktischer Bedeutung. 


\subsection{Umschlag im Bereich eines negativen Druckgra- dienten}

Durch die schrittweise Steigerung der Anströmungsgeschwindigkeit wird die lokale Profiltiefen-Reynoldszahl $R e_{x}$,

$$
R e_{x}:=U_{\infty} x_{c} / \nu
$$

an der Position 1 in Abbildung 6.1 bis zum Umschlagen der Grenzschicht erhöht. Vernachlässigt man Instabilitäten im Staulinienbereich, so stand die Grenzschicht bis zu dieser Position ausschließlich unter dem Einfluß der Querströmungsinstabilität: Die die Wandgrenzschicht begrenzende Potentialströmung ist bis dato beschleunigt und die Stromlinienkrümmung somit monoton. Resultat ist eine relativ dünne Grenzschicht mit ausgeprägter Querströmungskomponente, instabil gegen Querströmungsinstabilität. In Abbildung 6.2 sind die auf die Grenzschichtrandgeschwindigkeit normierten Beträge der Amplitudenspektren der Geschwindigkeitskomponenten $U_{s}$ und $V_{s}$ für verschiedene Anströmgeschwindigkeiten dargestellt. Während für die Grenzschichtrandgeschwindigkeit von $Q_{e}=35,7 \mathrm{~m} / \mathrm{s}$ noch eine klar laminare Grenzschicht vorlag, findet man bei $Q_{e}=43,8 \mathrm{~m} / \mathrm{s}$ eine turbulente Grenzschicht. Die Darstellungen der übrigen Geschwindigkeiten dokumentieren den Übergang.

Die Amplituden $U_{s}$ und $V_{s}$ haben für eine Frequenz die gleiche Größenordnung. Die größten Amplituden wurden für die tiefsten Frequenzen gemessen. Die transitionellen, normierten Spektren zeigen für Frequenzen $f<250 \mathrm{~Hz}$ keine Abhängigkeiten von der Anströmungsgeschwindigkeit. Oberhalb dieser Frequenz erkennt man ein Anwachsen der spektralen Amplituden. Es kommt zu einer Aufweitung des Störspektrums für höhere Grenzschichtrandgeschwindigkeiten.

Das Spektrum zur Grenzschichtrandgeschwindigkeit von $Q_{e}=43,8 \mathrm{~m} / \mathrm{s}$ nimmt eine Sonderrolle ein. Für diese Geschwindigkeit liegt eine turbulente Grenzschschichtströmung vor. Dieses Spektrum unterscheidet sich in den hochfrequenten Komponenten kaum von dem der transitionellen laminaren Grenzschicht für $Q_{e}=40,7$. Die tieffrequenten Fluktuationen sind allerdings deutlich geringer. Den Grad der Ausbildung der turbulenten Grenzschicht kann man in Abbildung 6.3 erkennen. Dort ist die Größe

$$
\left|S_{K o h}\right|=\frac{\left|\sqrt{S\left(U_{1, e f f}\right) \cdot S\left(U_{2, e f f}\right)}\right|}{Q_{e}}
$$

dargestellt. $U_{n, \text { eff }}$ bezeichnet dabei die effektive Kühlgeschwindigkeit eines einzelnen, geschwindigkeitskalibrierten Hitzdrahtes einer V-Sonde mit dem Index n, also die Geschwindigkeitskomponente des Geschwindigkeitsvektors, die den Hitzdraht kühlt. Die beiden Einzelhitzdrähte haben einen Mitte-Mitte-Abstand von circa einem Millimeter.

Bis zur Grenzschichtrandgeschwindigkeit von $40 \mathrm{~m} / \mathrm{s}$ werden die instationären Geschwindigkeitsfluktuationen dominiert von räumlich gut kohärenten Instabilitätswellen. Die Amplituden liegen dabei in der selben Größenordnung wie die der Spektren der $U_{s^{-}}$ Komponente. 

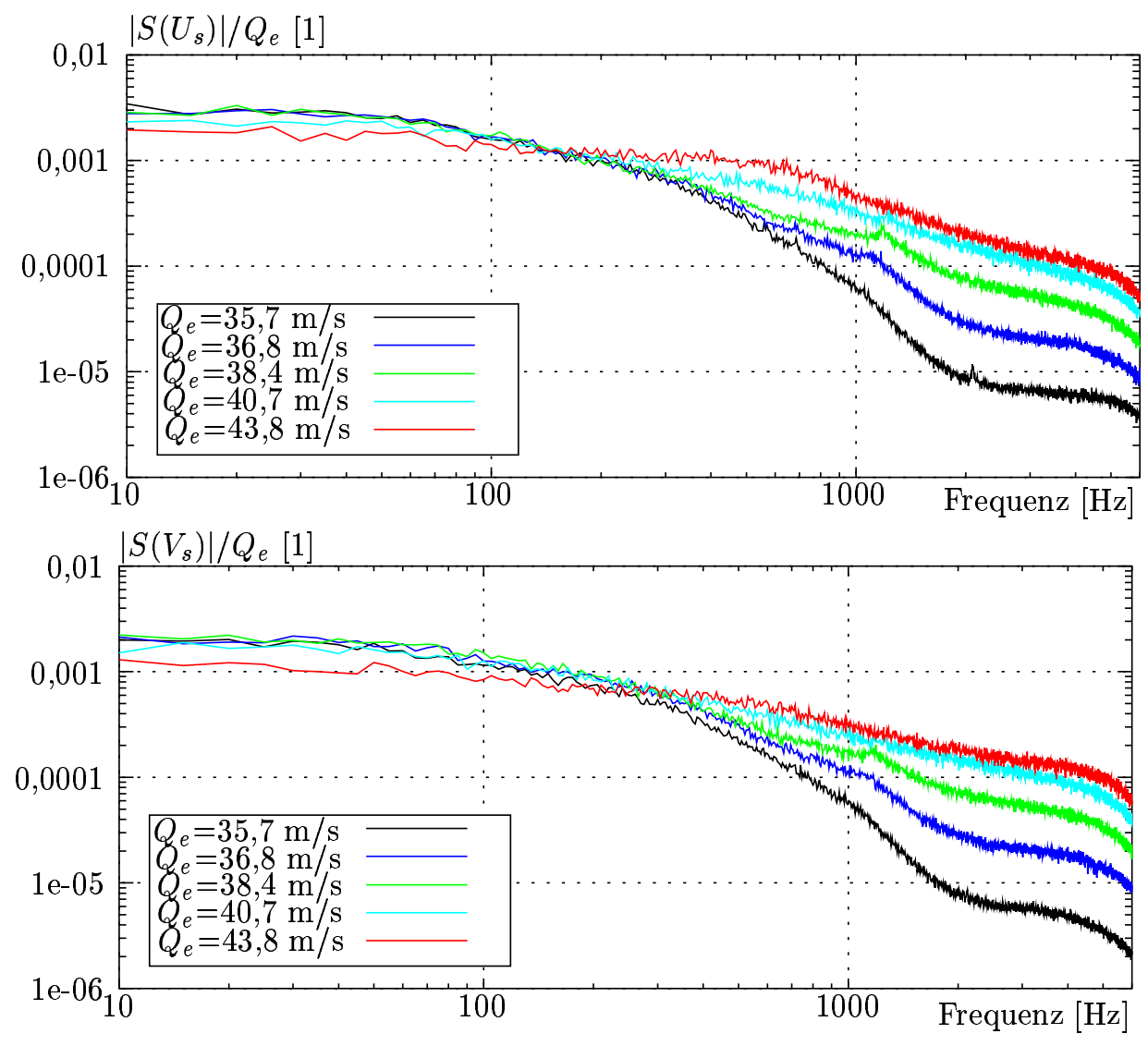

Abbildung 6.2: Entwicklung des Betrages der normierten Amplitudenspektren von $\left(U_{s}, V_{s}\right)$ während der Transition bei $x / c=0,35, z_{0}=z_{\max C F}$.

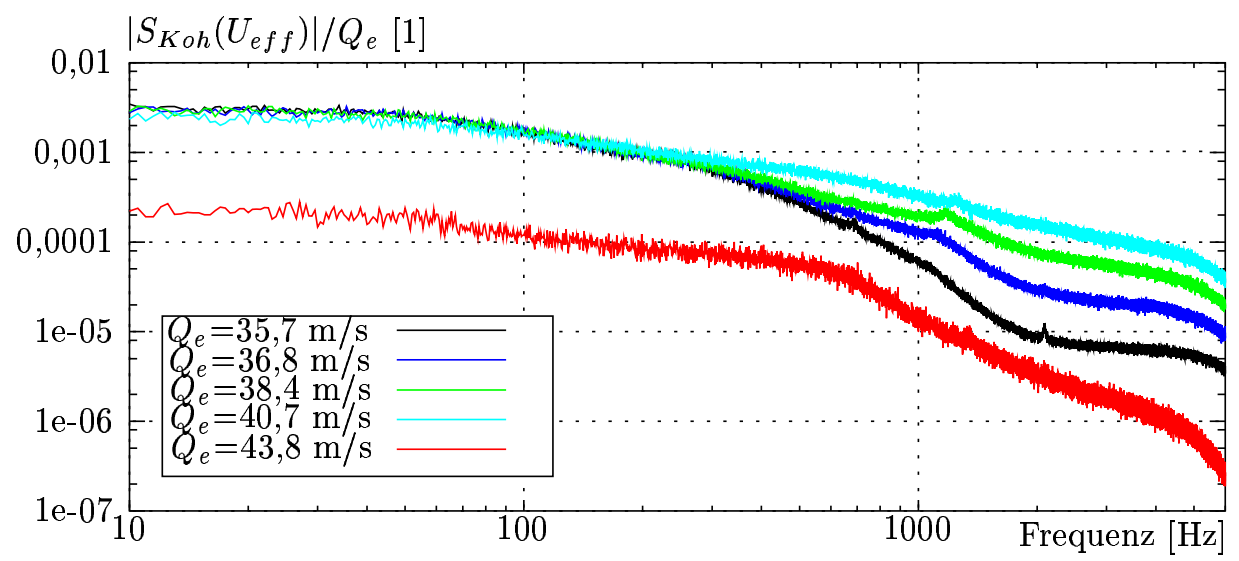

Abbildung 6.3: Entwicklung des Kreuzspektrums zwischen $U_{1, \text { eff }}$ und $U_{2, e f f},(\Delta s=1 \mathrm{~mm})$ während der Transition bei $x / c=0,35, z_{0}=z_{\max C F}$. 


\begin{tabular}{|l||l|l|l|l|l|}
\hline $\begin{array}{l}\text { Grenzschichtrandge- } \\
\text { schwindigkeit [m/s] }\end{array}$ & 35,7 & 36,8 & 38,4 & 40,7 \\
\hline$(U s-<U s>) / Q_{e}[1]$ & $\begin{array}{l}0,115 \quad \text { bei } \\
z_{0} / \delta_{99,9}=0,2\end{array}$ & $\begin{array}{l}0,14 \quad \text { bei } \\
z_{0} / \delta_{99,9}=0,18\end{array}$ & $\begin{array}{l}0,135 \quad \text { bei } \\
z_{0} / \delta_{99,9}=0,18\end{array}$ & $\begin{array}{l}0,09 \\
z_{0} / \delta_{99,9}=0,2\end{array}$ \\
\hline$R M S\left(U_{s}\right) / Q_{e}[1]$ & $\begin{array}{l}0,115 \quad \text { bei } \\
z_{0} / \delta_{99,9}=0,23\end{array}$ & $\begin{array}{l}0,13 \quad \text { bei } \\
z_{0} / \delta_{99,9}=0,23\end{array}$ & $\begin{array}{l}0,14 \\
z_{0} / \delta_{99,9}=0,18\end{array}$ & $\begin{array}{l}0,14 \quad \text { bei } \\
z_{0} / \delta_{99,9}=0,07\end{array}$ \\
\hline
\end{tabular}

Tabelle 6.1: Maximale rel. Amplituden der stationären spannweitigen Geschwindigkeitsmodulation und der RMS-Werte von $U s$ für verschiedene Grenzschichtrandgeschwindigkeiten.

Das Kreuzspektrum zur Grenzschichtrandgeschwindigkeit von $43 \mathrm{~m} / \mathrm{s}$ liegt im Frequenzbereich der primär angefachten Instabilitäten $(f<2000 \mathrm{~Hz})$ in der Amplitude etwa eine Größenordnung unter dem der anderen Randgeschwindigkeiten. Hier dominieren die für eine turbulente Grenzschicht typischen kleinskalige Geschwindigkeitsschwankungen die instationären Anteile.

Die in diesem Abschnitt bisher dargestellten Ergebnisse wurden durch ein spannweitiges Mitteln der jeweiligen Auswertungsgrößen gewonnen. Dabei vernachlässigt man, daß zu diesem späten Zeitpunkt der Grenzschichtentwicklung schon Interaktionen zwischen den einzelnen Störungsformen stattfinden. Besonders auffällig ist diese Interaktion zwischen stationärer spannweitiger Modulation und den instationären Anteilen, die im RMS-Wert zusammengefaßt werden.

Abbildung 6.4 stellt die seitliche Ansicht auf eine Profilreihe dar. Jeweils gegenüber gestellt sind die $U_{s}$-Geschwindigkeitskomponente und der RMS-Wert für Profilmessungen verschiedener spannweitiger Positionen.

Zunächst kann man die stationären und instationären maximal erreichten Amplituden abschätzen. Eine Zusammenfassung dieser Werte findet man in Tabelle 6.1. Ein Vergleich mit den von Müller [48] und Lerche [14] experimentell ermittelten Werten (gemessen an einem Modell einer schiebenden Platte mit aufgeprägten Druckgradient) offenbart für die stationären Amplituden im vorliegenden Fall relativ kleine Werte: maximal ergab sich eine Amplitude von $14 \%$ bei einer Anregung mit Rauhigkeitselementen der Höhe $20 \mu m$, Müller gibt $20 \%$ an (selber Kanal), Lerche [14] ermittelte für den Fall der Anregung stationärer Störungen ebenfalls $20 \%$, allerdings gemessen in einem Kanal mit höherem Turbulenzgrad. Die Ursache für diese relativ kleinen stationären Amplituden könnten in der konvexen Oberflächenkrümmung des Modells liegen. Aufschluß darüber können aber erst nichtlineare Rechnungen liefern, die bisher noch nicht vorliegen.

Die von mir ermittelten maximalen RMS-Werte der Geschwindigkeitskomponente $U_{s}$ liegen ebenfalls bei $14 \%$, bestimmt in einem Frequenzbereich von $0,5 \mathrm{~Hz}<f<6000 \mathrm{~Hz}$. Damit liegen sie höher als die von Deyhle [47] (11 \%) und Lerche (10\%, unabhängig von der Anregung stationärer und/oder instationärer Störungen), beide aufgenommen im 
1-m-Kanal Göttingen. Auch hier kann eine lineare Theorie keine schlüssige Erklärung liefern. Die Darstellungen offenbaren zwei lokale Maxima im Abstand von ca. $z / \delta_{99,9}=0,2$ und $z / \delta_{99,9}=0,7$, wobei das wandnähere Maximum eine höhere Amplitude besitzt. Das reproduziert die Ergebnisse von Lerche, gewonnen durch die kontrollierte Anregung instationärer Störungen. Unterschiedlich ist jedoch das Amplitudenverhältnis der beiden Maxima zueinander und die Entfernung des äußeren Maximums zur Wand. Ebenso bestätigt wird die spannweitige Modulation der Amplitude, korrespondierend zur Lage der stationären Querströmungswirbel.

Die beiden Darstellung in Abbildung 6.4 für die Grenzschichtrandgeschwindigkeit von $Q_{e}=40,7 \mathrm{~m} / \mathrm{s}$ weisen Besonderheiten hinsichtlich der eben diskutierten Abhängigkeiten auf. Im zeitlich gemittelten Profil erkennt man einen Rückgang der spannweitigen Modulation und in der Darstellung des RMS-Wertes entstand ein weiteres (lokales) Maximum in Wandnähe. Darüber hinaus fällt der RMS-Wert zum Grenzschichtrand deutlich schwächer ab. Die Ursache dafür ist das nun häufige Auftreten turbulenter Spots.

Die Wechselwirkung der instationären Strömungsanteile mit den stationären Querströmungswirbeln und der zeitlich gemittelten Geschwindigkeit entnimmt man der Abbildung 6.5. Dort ist deren Entwicklung für vier verschiedenene Grenzschichtrandgeschwindigkeiten dokumentiert. Bei $\left(y_{c}=4 m m, z / \delta=0,5\right)$ befindet sich ein Wendepunkt im $U_{s^{-}}$ Profil. Dieses Geschwindigkeitsprofil ist links zusammen mit dem spannweitig gemittelten Geschwindigkeitsprofil $\left\langle U_{s}>\right.$ dargestellt. Die Grenzschichtrandgeschwindigkeit nimmt von oben nach unten zu.

Generell stellt man fest, daß die RMS-Werte ein in Spannweitenrichtung breites und in Wandabstandsrichtung schmales Maximum im Bereich des Minimums von

$\left(U_{s}-<U_{s}>\right)$ aufweisen. Neben dieser Grobstruktur existieren noch weitere kleinere Strukturen. Ein lokales RMS-Maximum befindet sich in der Umgebung der Wendepunkte von $U_{s}$. Zu ihnen verläuft ein schmaler Grat den Rand des Unterströmungsgebietes entlang. Besonders für die Grenzschichtrandgeschwindigkeiten von $Q_{e}=36,8 \mathrm{~m} / \mathrm{s}$ und $Q_{e}=38,4 \mathrm{~m} / \mathrm{s}$ erkennt man, daß sich das breite RMS-Maximum zwischen zwei lokalen Maxima an den Rändern der Untergeschwindigkeitsgebiete von $U_{s}$ aufspannt.

Eine gewisse Sonderrolle in dieser Abfolge von Grenzschichtprofilreihen nimmt die Grenzschichtrandgeschwindigkeit $Q_{e}=40,7 \mathrm{~m} / \mathrm{s}$ ein. Zunächst fällt auf, daß sich das zeitlich gemittelte Geschwindigkeitsfeld verändert hat. Das zentrale Untergeschwindigkeitsgebiet $\left(4 m m<y_{c}<6 m m\right)$ hat seinen Wandabstand vergrößert und wird von den benachbarten Übergeschwindigkeitsgebieten gewissermaßen unterlaufen. Bemerkenswert ist die Entstehung eines globalen Maximums des RMS-Wertes in unmittelbarer Wandnähe unterhalb des Untergeschwindigkeitsgebietes. Leider existieren keine Profilreihenuntersuchungen für Grenzschichtrandgeschwindigkeiten oberhalb von $Q_{e}=40,7 \mathrm{~m} / \mathrm{s}$.

Der wichtigste Unterschied zwischen den Ergebnissen am Flügel mit konvex gekrümmter Oberfläche und den Ergebnissen an der schiebenden Platte (Lerche [14]) liegt in der Tatsache, daß im vorliegenden Fall die Transition ohne die sogenannten hochfrequenten sekundären Instabilitäten stattfand. Lerche detektierte in der Umgebung des Wendepunkts 

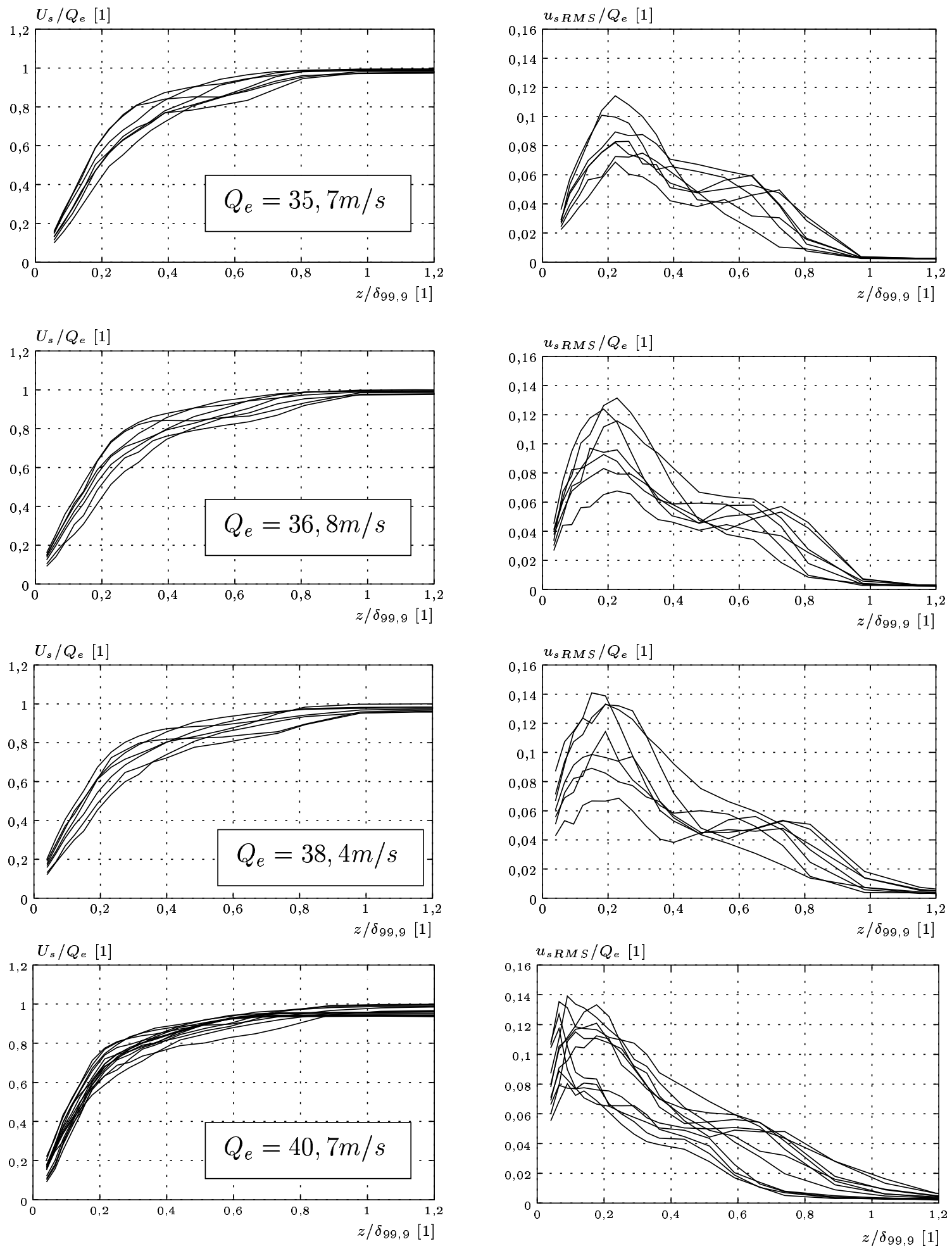

Abbildung 6.4: Entwicklung des RMS-Wertes von $U_{s}$ während der Transition bei $x / c=0,35$. 

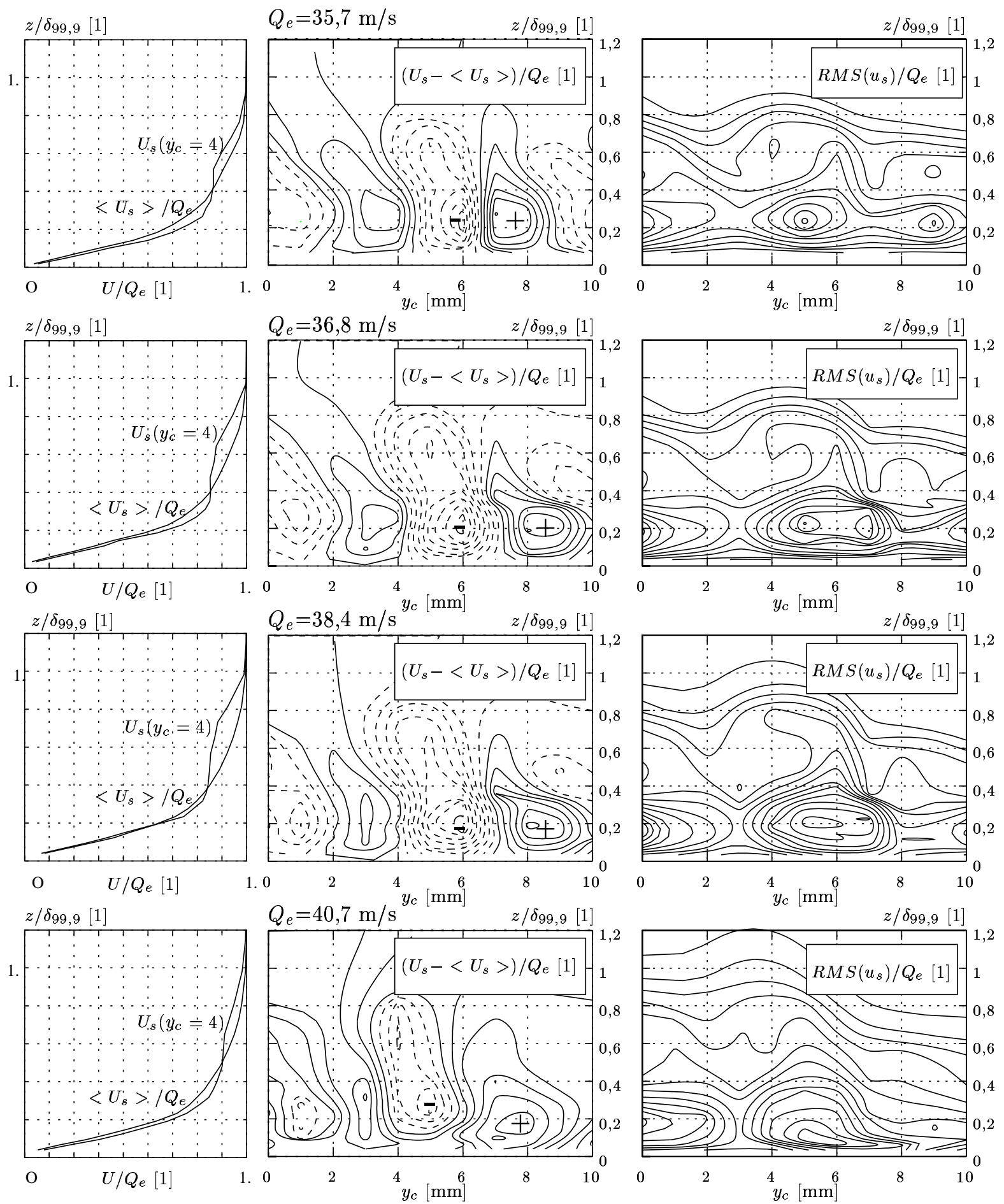

Abbildung 6.5: $\left\langle\bar{U}_{s}>\right.$ sowie Wendepunktsprofil (Links), Isolinien der stationären spannweitigen Modulation der spannweitig und zeitlich gemittelten Geschwindigkeitsprofile (Mitte) und der korrespondierenden RMS-Wert- Konturen für verschiedene Grenzschichtrandgeschwindigkeiten an der Position $x / c=0,35$ (Rechts). Linienabstand der Konturdiagramme: $1 \% Q_{e}$. 
von $U_{s}$ das Auftreten von Geschwindigkeitsfluktuationen in einem um eine Größenordnung höheren Frequenzband als die der primär angefachten Instabilitäten. Diese Störungen konnten als sekundäre, d.h. erst durch die Wirkung primärer Instabilitätsmechanismen hervorgerufener Instabilitäten, interpretiert werden. Sie traten unmittelbar vor dem Zusammenbruch der laminaren Grenzschicht auf. Die Ursache für das Fehlen dieser Folge sekundärer Instabilitäten ist mutmaßlich in dem im vorliegenden Experiment nur sehr schwach ausgeprägten Wendepunkt von $U_{s}$ zu suchen, der aus dem schwachen Wachstum der stationären Wirbelstörungen resultiert. Ich werde im nächsten Abschnitt näher auf das Phänomen der sekundären Instabilität eingehen.

\subsection{Umschlag im Bereich eines positiven Druckgradi- enten}

Das im vorigen Abschnitt beschriebene Umschlagsszenario fand einzig unter Mitwirkung der Querströmungsinstabilität statt. Für technische Anwendungen, wie die Tragflügelumströmung moderner Verkehrsflugzeuge, ist neben dieser Reinform auch ein Umschlagszenario interessant, bei dem verschiedene Instablilitäten beteiligt sind. Hier existieren bisher nur wenige experimentelle Untersuchungen, zum Beispiel von Bippes ([49], [50]) an einem schiebend zur Anströmung angeordneten Profil mit einem zunächst konkav, dann konvex gekrümmten Segment, dessen Grenzschicht zunächst Görtler-instabil und dann querströmungsinstabil war.

In diesem Abschnitt wird der Umschlag der Grenzschicht an Position 2 (vgl. Abbildung 6.1 auf Seite 69) untersucht. Bis zum Erreichen des Druckminimums bei $x / c=0,38$ liegt zunächst eine beschleunigte Wandgrenzschicht vor. In Verbindung mit der Stromlinienkrümmung herrscht bis zu diesem Punkt einzig die Querströmungsinstabilität. Durch die Wahl einer geringeren Anströmungsgeschwindigkeit kommt es allerdings noch nicht zum laminar-turbulenten Umschlag der Wandgrenzschicht. Nach Erreichen des Druckminimums wird die Grenzschicht sehr schnell dicker, wie man in Abbildung 3.5 auf Seite 29 an Hand der Entwicklung der Verdrängungsdicke erkennen kann. Durch die Wirkung der Querströmungsinstabilität ist an der Profiltiefenposition 2 die Grundströmung bereits stark deformiert (Abbildung 5.1 auf Seite 62). Ebenso liegen große Amplituden der stationären Querströmungswirbel (Abbildung 4.9 auf Seite 45) sowie der instationären Störungen (Abbildung 4.18 auf Seite 56) vor. In diesem Zustand kommt als weitere Instabilität die Tollmien-Schlichting Instabilität ins Spiel.

Unter diesen Bedingungen wird im vorliegenden Experiment das Auftreten einer hochfrequenten Instabilität, ähnlich den sekundären Instabilitäten an der schiebenden Platte, beobachtet. Diese beeinflusst den Zusammenbruch der laminaren Strömung in besonderem Maße. Es soll daher im folgenden versucht werden, Bedingungen für das Auftreten und die Wirkung auf den Umschlag am konvexen Teil des Flügels zu ermitteln. 

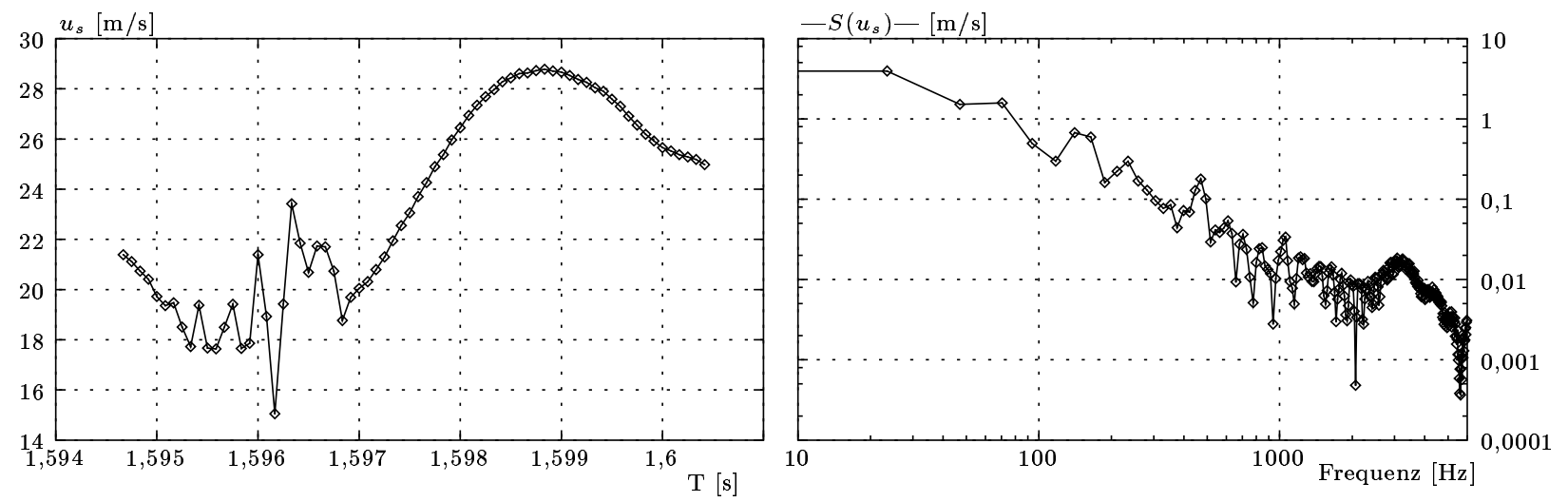

Abbildung 6.6: Geschwindigkeitsverlauf $U_{s}$ und Betrag des Amplitudenspektrums in der Umgebung einer hochfrequenten sekundären Instabilität.

\subsubsection{Hochfrequente Instabilitäten}

Hochfrequente Geschwindigkeitschwankungen, wie die in Abbildung 6.6 dargestellte, traten im vorliegenden Experiment zu zufälligen Zeiten, an mehr oder weniger zufälligen Orten und über zufällige Zeiträume auf. Um eine physikalische Interpretation dieser Erscheinungen geben zu können, mußten sie zuächst sicher erkannt und dann nach bestimmten Parametern wie Häufigkeit ihres Auftretens, Amplitudenentwicklung, räumliche Kohärenzen und Ausbreitungsrichtungen in der Grenzschicht klassifiziert werden. Schließlich wird versucht, sie im Kontext einer Instabilität zu diskutieren.

\section{Identifikation}

Zunächst war es nötig, die spontan auftretenden und nur kurz anhaltenden Geschwindigkeitsfluktuationen aus den aufgenommenen Meßsignalen zu extrahieren. Bei der Identifikation hochfrequenter Geschwindigkeitsschwankungen macht man sich die experimentell beobachtete Eigenschaft zu nutze, daß sich das Störspektrum vergleichsweise plötzlich ändert. Diese plötzliche Änderung der Geschwindigkeitsfluktuation findet man sowohl in der Änderung der Geschwindigkeit (erste Ableitung von $\vec{u}$ nach der Zeit) als auch in der Änderung der Änderung der Geschwindigkeit (zweite Ableitung von $\vec{u}$ nach der Zeit). In Verbindung mit der geeigneten Wahl eines Schwellenwertes der zweiten Ableitung des Betrages der Geschwindigkeit $|u|$ läßt sich mit diesem Verfahren die Detektion der hochfrequenten Geschwindigkeitsschwankungen sicher automatisieren.

\section{Klassifikation}

Wie bereits für die Störungen in Folge primärer Instabilitäten sollte auch für die hochfrequenten Geschwindigkeitsschwankungen versucht werden, einige Parameter wie Wel- 


\begin{tabular}{|l||l|l|}
\hline & Mode 1 & Mode 2 \\
\hline \hline Vorkommen & untere Grenzschicht & mittlere Grenzschicht \\
\hline Ausbreitungsrichtung & $\Phi_{s}=0^{\circ}$ & $\Phi=25^{\circ}$ \\
\hline Wellenlänge & $1,7 \mathrm{~mm}$ & $1,7 \mathrm{~mm}$ \\
\hline Typische Frequenzen & $1700-2100 \mathrm{~Hz}$ & $2000-3000 \mathrm{~Hz}$ \\
\hline Phasengeschwindigkeit & $(2000 \mathrm{~Hz}) \cdot 1,7 \mathrm{~mm}=3,4 \mathrm{~m} / \mathrm{s}$ & $(2300 \mathrm{~Hz}) \cdot 1,7 \mathrm{~mm}=3,9 \mathrm{~m} / \mathrm{s}$ \\
\hline
\end{tabular}

Tabelle 6.2: Eigenschaften beobachteter hochfrequenter Geschwindigkeitsfluktuationen.

lenlänge, Ausbreitungsgeschwindigkeit und Ausbreitungsrichtung zu ermitteln.

Die Benutzung der Begriffe Wellenlänge, Ausbreitungsrichtung und Ausbreitungsgeschwindigkeit impliziert die Annahme, daß die beobachteten hochfrequenten Geschwindigkeitsfluktuationen (Instabilitäts-)Wellen sind. Um diese Annahme zunächst zu prüfen, wurde die räumliche Korellation, also die räumliche Phasenkopplung der Geschwindigkeitsfluktuationen, untersucht. Dazu sind in Abbildung 6.7 einige Beispiele der an beiden Hitzdrähten der V-Sonde gemessenen effektiven Kühlgeschwindigkeiten zusammengefaßt. Die angegebenen Beispiele sind repräsentativ für die gefundenen hochfrequenten Schwankungen.

Nur zwei der 12 angegebenen Beispiele zeigen für nur einen Hitzdraht eine hochfrequente Modulation der effektiven Kühlgeschwindigkeit und damit keine spannweitige Korellation. Die übrigen Beispiele zeigen eine relativ konstante Phasenbeziehung zwischen den beiden Einzelhitzdrähten. Daraus kann man die Vermutung ableiten, daß es sich bei der Mehrheit der detektierten Geschwindigkeitsschwankungen um wellenartige handelt. Ihre typische spannweitige Ausdehnung ist größer als ein Millimeter, dem spannweitigen Abstand der beiden Einzelhitzdrähte. Die Phasenbeziehungen der verschiedenen hochfrequenten Modulation benachbarter Hitzdrähte sind nicht einheitlich. Die Hälfte der dargestellten Beispiele weisen eine von Null verschiedene Phasenlage zwischen den beiden Kühlgeschwindigkeiten auf (Phasenunterschiede bis ca. 90 Grad), ein Drittel ist gleichphasig. Die gleichphasigen Ereignisse treten signifikant häufiger in Wandnähe auf.

Mit der noch zu prüfenden Annahme, daß es sich bei den zwei beobachteten Phasenunterschieden um zwei verschiedene Moden handelt, kann man durch Veränderung des Sondenwinkels für beide Moden die in Tabelle 6.2 dargestellten Parameter ermitteln. Die dazu verwendeten Verfahren sind dabei identisch zu denen, die in den Abschnitten 4.4.1 auf Seite 51 bzw. 4.4.1 auf Seite 49 beschrieben wurden.

Die Berechtigung dieser Annahme wird gegenwärtig in Experimenten mit kontrollierter Anregung hochfrequenter Störungen und begleitenden sekundären Instabilitätsanalysen geprüft. Es bleibt die Feststellung, daß es sich bei den hochfrequenten Geschwindigkeitsf- 

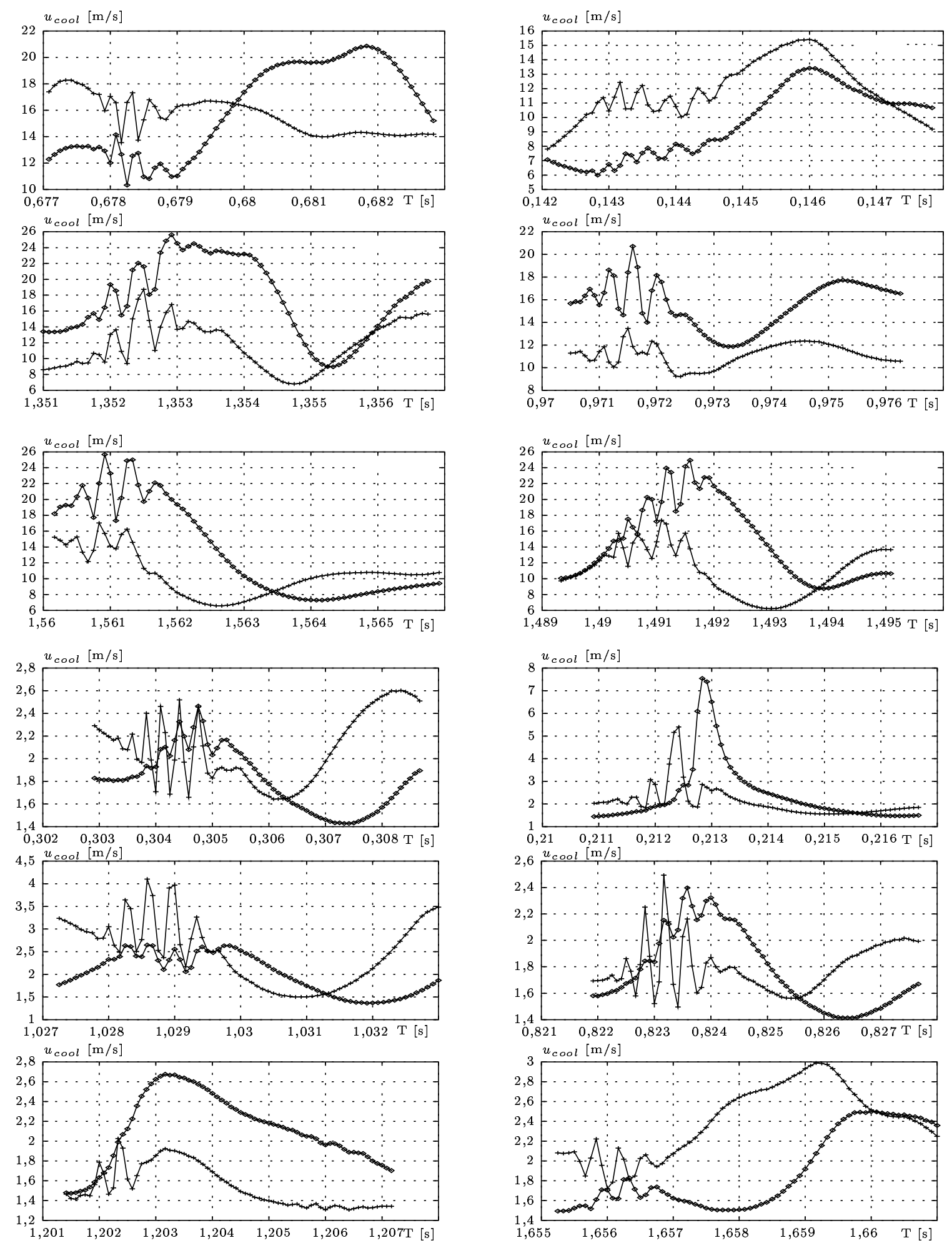

Abbildung 6.7: Effektive Kühlgeschwindigkeiten der beiden Einzelhitzdrähte in der Umgebung hochfrequenter Geschwindigkeitsfluktuationen. 
luktuationen um wellenförmige Störungen handelt.

\section{Verteilung in der Grenzschicht}

Weitere Anhaltspunkte zur Ursache der hochfrequenten Geschwindigkeitsfluktuationen können die Positionen in der Grenzschicht geben, an denen sie gehäuft auftritt. Dahinter steckt die Idee, daß es sich bei den detektierten Störungen um die Folge sekundärer Instabilitäten in der durch primär angefachte Störungen nunmehr veränderten Grenzschichtströmung handelt.

Zur Verdeutlichung der gegebenen Strömungssituation an der im folgenden betrachteten Profiltiefenposition $x / c=0,425$ sind in der Abbildung 6.8 die zeitlich gemittelten Strömungsgeschwindigkeiten und die RMS-Werte dargestellt. In der Darstellung erkennt man zunächst den deutlich ausgebildeten stationären Wirbel der Wellenlänge $\lambda=6 \mathrm{~mm}$ und der Amplitude von $15 \%$ in $U_{s}$. Bei $y_{c}=93 \mathrm{~mm}$ erkennt man einen gut ausgeprägten Wendepunkt im $U_{s}$ - Profil, dessen zugehöriges Profil mit dargestellt ist, (bei $y_{c}=87 \mathrm{~mm}$ ist dieser Wendepunkt zwar angedeutet, aber nicht ausgeprägt). Da infolge des sich umkehrenden Druckgradients die Richtung der Querströmungsgeschwindigkeit sich zu ändern beginnt, findet man negative Werte der zeitlich gemittelten Querströmungsgeschwindigkeitskomponente $V_{s}$ in Wandnähe. Die durch die stationären $\lambda_{y_{c}}=6 \mathrm{~mm}$-Längswirbel im Stromaufbereich verursachte Modulation der $V_{s}$ - Komponente ist auch an dieser Profiltiefenposition präsent. Folgt man den experimentellen Ergebnissen hinsichtlich der Amplitudenentwicklung stationärer Störungen oder den linearen Theorien mit Krümmungsberücksichtigung (vergl. Abbildung 4.9 auf Seite 45), so sind stationäre Störungen nicht mehr angefacht.

Die RMS-Werte sind, entsprechend den an dieser Position nichtlinearen Störungswechselwirkungen, spannweitig stark moduliert. Ihre maximalen Werte erreichen die RMS-Werte der beiden Strömungskomponenten an den spannweitig steilen Flanken der zugehörigen Geschwindigkeitskomponente. $U_{s, R M S}$ bildet dabei an beiden Flanken je ein Maximum aus, $V_{s, R M S}$ nur an einer Flanke. In der Umgebung des Wendepunktes von $U_{s}$ sind beide RMS-Wert-Komponenten mit $U_{s, R M S}=4 \%$ bzw. $V_{s, R M S}=2 \%$ nur gering ausgeprägt.

Die Orte mit vermehrten Auftreten hochfrequenter Geschwindigkeitsschwankungen sind durch die grau schraffierten Kreise angedeutet. Man findet sie insbesondere an Stellen mit quasi nicht vorhandener Querströmungskomponente. Die $U_{s}$ - Profile weisen an dieser Stelle keine Wendepunkte auf.

In Abbildung 6.9 sind die Resultate für Traversenmessungen bei konstanter Höhe $z=$ 0, 56 für eine Kaskade von Anströmungsgeschwindigkeiten dargestellt. In der linken Hälfte aller Darstellungen findet man ein Gebiet mit nicht ausgeprägten stationären Störungen. Die RMS-Werte in diesem Gebiet sind mit $10 \%$ der Anströmungsgeschwindigkeit nicht zu vernachlässigen. Allerdings findet man in diesem Gebiet eine nur sehr schwache Tendenz zur Ausbildung sekundärer Instabilitätsereignisse. Das Gebiet auf der rechten Seite der Darstellungen ist identisch mit dem in Abbildung 6.8 dargestellten. 

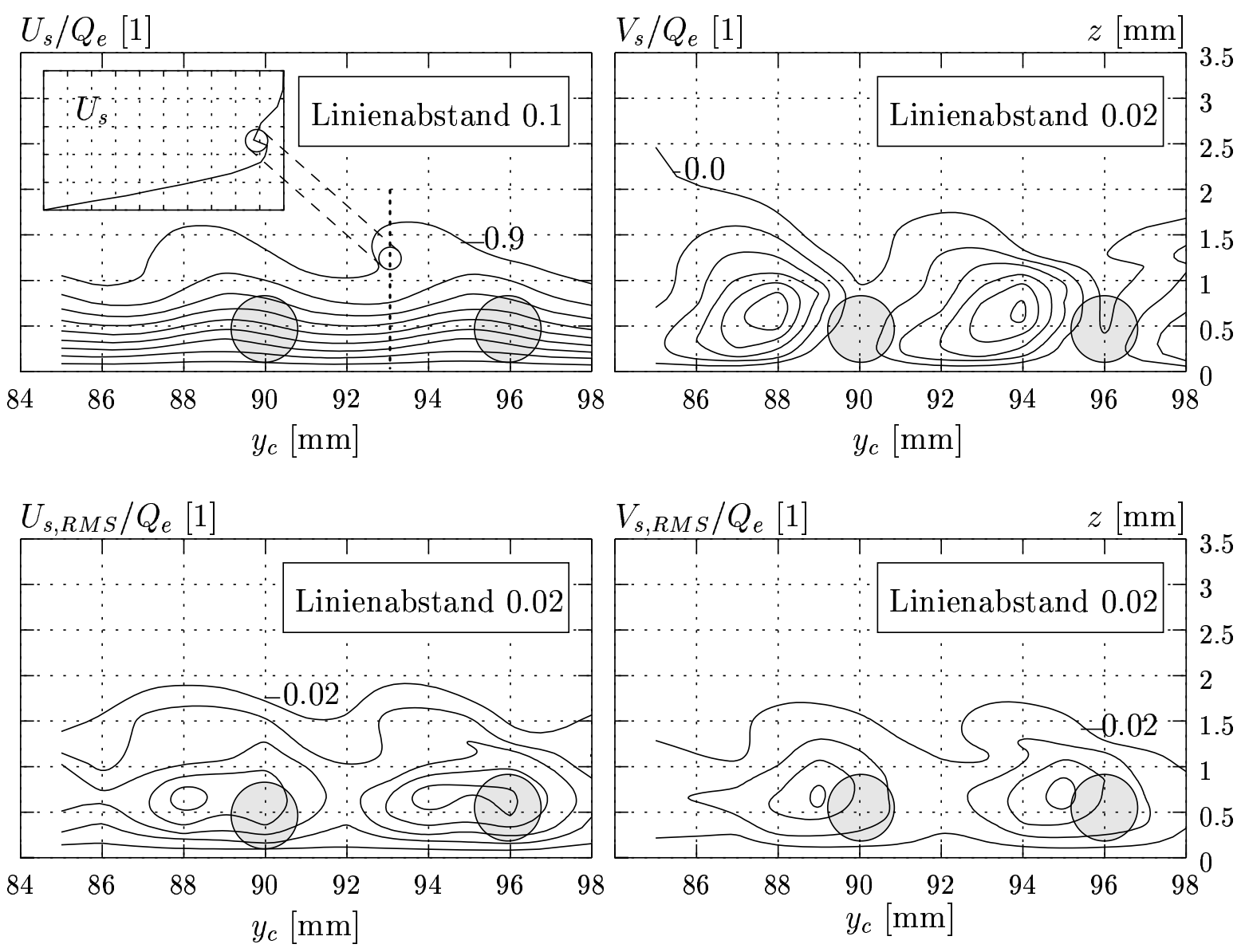

Abbildung 6.8: Gestalt der zeitl. gemittelten Grenzschicht und der RMS-Werte bei $x_{c} / c=$ $0,425, U_{\infty}=28 \mathrm{~m} / \mathrm{s}$. Die Kreise bezeichnen das Zentrum der maximalen Häufigkeit hochfrequenter Störungen.

Mit dem Einsetzen der stationären Störungen ab $y_{c}=82 \mathrm{~mm}$ zeigen sich bei allen Anströmungsgeschwindigkeiten deutliche Abhängigkeiten der RMS-Werte und der Anzahl der hochfrequenten Geschwindigkeitsfluktuationen, sowohl von der spannweitigen Position als auch von der Anströmungsgeschwindigkeit.

Der dominante stationäre Querströmungswirbel ist für alle betrachteten Anströmungsgeschwindigkeiten in der Sättigung, er stagniert bzw. wird in seiner Amplitude sogar kleiner $\left(U_{\infty}=33 m / s\right)$.

Die RMS-Werte bilden zunächst eine um $180 \mathrm{Grad}$ zu $U_{s}$ phasenverschobene Modulation aus, überlagert von Nebenmaxima an den steilen Flanken der $U_{s}$ - Periodizität. Bei $U_{\infty}=32 \mathrm{~m} / \mathrm{s}$ erreicht auch die Amplitude der RMS - Modulation ein Maximum. Die Maxima an den Flanken von $U_{s}$ sind weniger deutlich bzw. verschwunden. Bei der Anströmungsgeschwindigkeit von $33 \mathrm{~m} / \mathrm{s}$ sind die RMS-Werte an einigen spannweitigen Posi- 
tionen rückläufig, die Grenzschicht ist dort bereits turbulent, während sie in den benachbarten Bereichen noch laminar ist. Diese Umschlagsform ist typisch für ein Umschlagszenario infolge von Querströmungsinstabilität ${ }^{1}$.

Mit der Ausprägung der spannweitigen RMS - Modultation beginnen im Gebiet mit starker stationärer Mode vermehrt hochfrequente Geschwindigkeitsfluktuationen aufzutreten. Die maximale Anzahl dieser Ereignisse detektiert man im Bereich der Minima von $U_{s}$, also in Phase zur RMS - Modulation. Allerdings kommt es hier nicht zur Ausbildung von Nebenmaxima wie bei jener. Die Anzahl der Ereignisse wächst mit steigender Anströmungsgeschwindigkeit weiter, auch wenn die RMS - Werte stagnieren.

Leider existieren keine Profilreihenuntersuchungen in ähnlicher Geschwindigkeitsaufösung für diese Profiltiefenposition. Deshalb ist auch keine Aussage darüber möglich, ob sich in der Umgebung des Wendepunkts im $U_{s}$ - Profil (vgl. Abbildung 6.8) eine Häufung hochfrequenter Geschwindigkeitsfluktuationen ergab, wie sie im DLR-Prinzipexperiment an der schiebenden Platte beobachtet worden sind. Allerdings sind die Verhältnisse zwischen beiden Experimenten in wesentlichen Punkten nicht unbedingt vergleichbar. Der RMS-Wert in der Umgebung der Wendepunkte ist im vorliegenden Experiment deutlich kleiner als beim Prinzipexperiment, und somit sind auch die momentanen Geschwindigkeitsprofile weniger deformiert als im Prinzipexperiment. Und nach Lerche sind diese verantwortlich für das Entstehen der sekundären hochfrequenten Instabilität. Ebenso hat man im vorliegenden Fall neben der Querströmungsinstabilität auch noch eine TollmienSchlichting Instabilität zu berücksichtigen.

Zusammenfassend kann man sagen, daß das Vorkommen hochfrequenter Geschwindigkeitsschwankungen sowohl von der zeitlich gemittelten Strömung (also der Amplitude der stationären Wirbel und der (0,0)-Mode) als auch von der RMS-Wert-Verteilung in der Grenzschicht, also der Amplitude der instationären Störungen, abhängt. Die Position ihres gehäuften Auftretens ist verschieden von der im DLR Prinzipexperiment festgestellten. Inwiefern sie evtl. eine Folge der Tollmien - Schlichting Instabilität der Grenzschicht ist, müssen numerische Stabilitätsanalysen der Grenzschicht zeigen.

\footnotetext{
${ }^{1}$ Visualisiert man den Umschlag (z.B. mit Infrarotaufnahmen der Profiloberflächen) so erhält man im Falle von transitionellen Grenzschichten mit Querströmungsinstabilität typischerweise eine ausgefranste spannweitige Umschlagslinie
} 

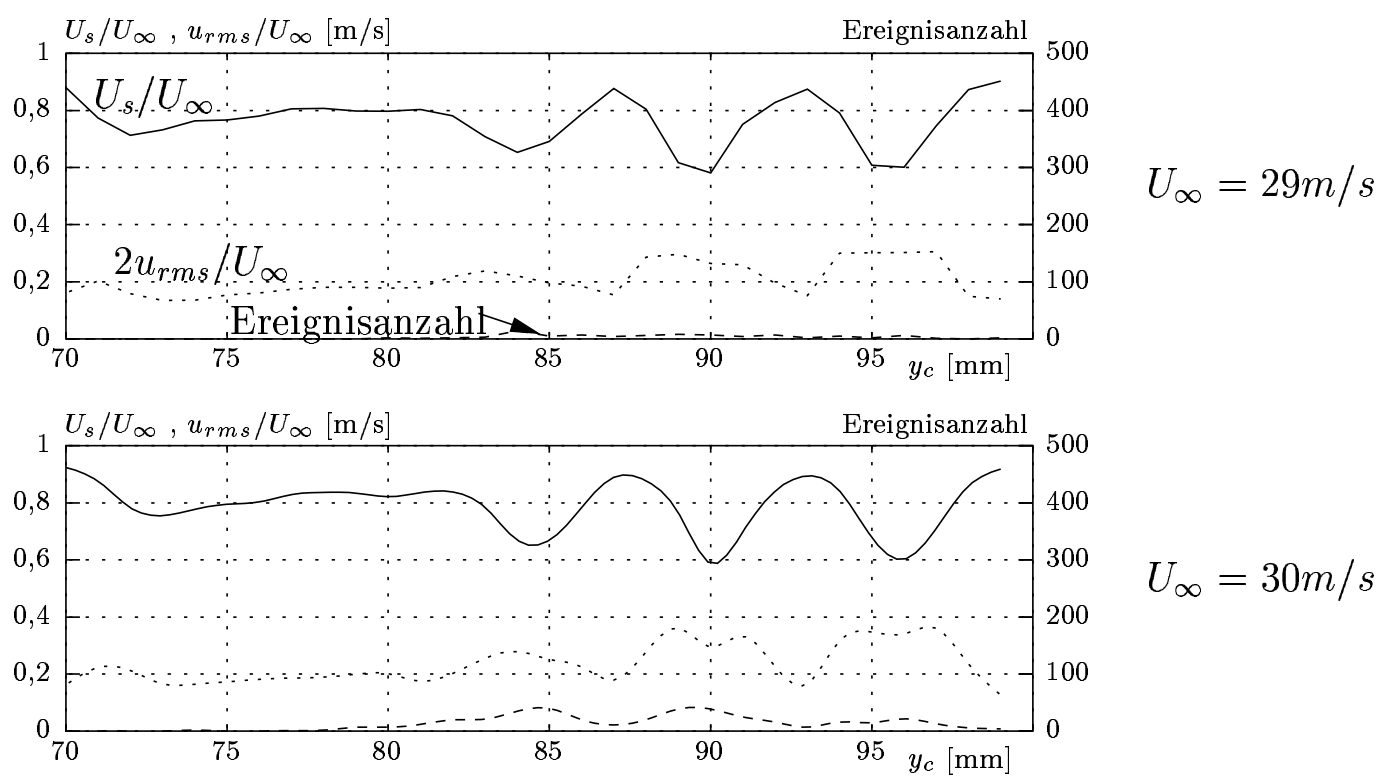

$$
U_{\infty}=30 \mathrm{~m} / \mathrm{s}
$$

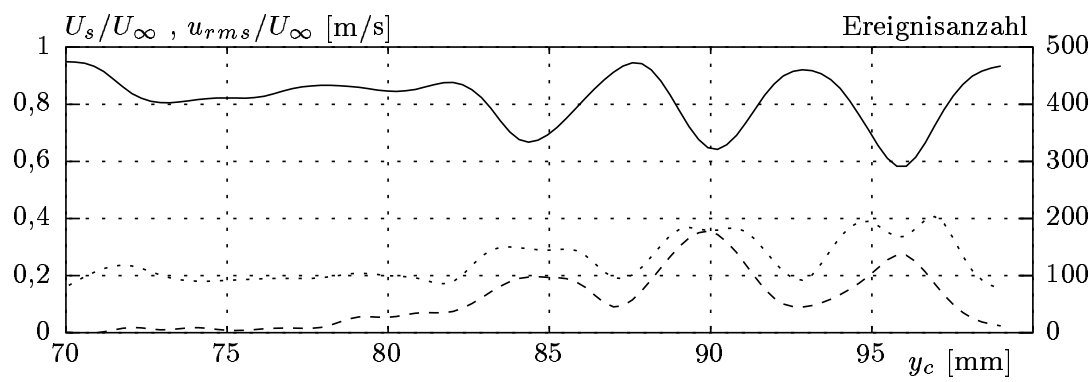

$$
U_{\infty}=31 \mathrm{~m} / \mathrm{s}
$$

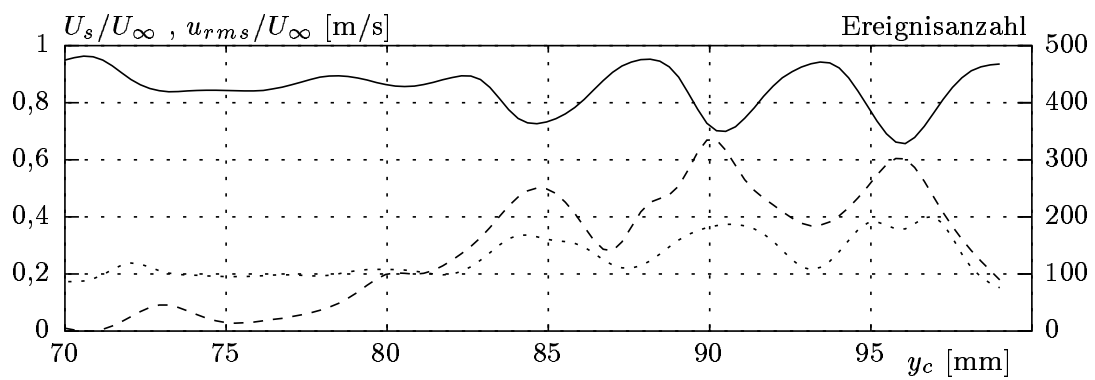

$$
U_{\infty}=32 \mathrm{~m} / \mathrm{s}
$$

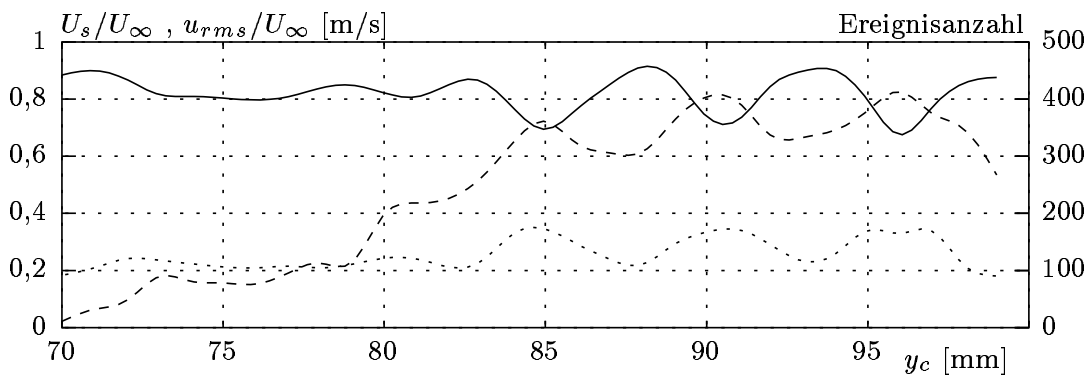

$$
U_{\infty}=33 \mathrm{~m} / \mathrm{s}
$$

Abbildung 6.9: Zusammenhang von $U_{s}, U_{r m s}$ und der Anzahl hochfrequenter Geschwindigkeitsschwankungen über einen Zeitraum von 2 Sekunden $\left(x_{c} / c=0,425, z=0,56 \mathrm{~mm}\right)$. 


\section{Kapitel 7}

\section{Zusammenfassung und Ausblick}

Die vorliegende, experimentelle Arbeit beschäftigt sich mit der Entwicklung einer laminaren, inkompressiblen, quasi-zweidimensionalen Grenzschichtströmung über einer konvex gekrümmten Oberfläche und ihrem laminar-turbulentem Übergang. Die betrachtete Grenzschichtströmung unterliegt dabei einem nichtkonstanten Druckgradienten, der die Grenzschichtströmung zunächst über einem weiten Bereich beschleunigt und schließlich, nach Durchlaufen eines Druckminimums, verzögert. Die Entwicklung von Potential - und Grenzschichtströmung wurde dokumentiert und mit den Ergebnissen von numerischen Strömungsberechnungsverfahren verglichen. Dabei zeigte sich eine gute Übereinstimmung beider, eine wichtige Voraussetzung für die folgenden Untersuchungen zur Störungsentwick-

lung in der Grenzschicht, insbesondere im Hinblick auf die Modellierung und Überprüfung von Stabilitätsberechnungsverfahren.

Im beschleunigten Bereich ist die betrachtete Grenzschicht primär querströmungsinstabil. Dadurch kommt es zunächst zur Ausbildung stationärer Querströmungswirbel und instationärer schräg laufender wellenartiger Störungen. Die Störungsentwicklung wurde für die stationären Querströmungswirbel dokumentiert und die Amplitudenentwicklung mit Berechnungen auf der Basis linearer Stabilitätsanalysen verglichen. Dabei offenbarte sich ein langer Bereich linearen Wachstums. Der Effekt der Oberflächenkrümmung auf die Störungsamplitudenentwicklung konnte durch die parallel durchgeführten linearen Stabilitätsanalysen als stabilisierend ermittelt werden. Er wurde jedoch durch die destabilisierende Wirkung der Nichtparallelität der Grenzschichtströmung für das konkrete Experiment wieder teilweise kompensiert.

Zur Beschreibung der Entwicklung instationärer Störungen wurden Auswerteverfahren entwickelt, die es trotz der zunächst sehr kleinen Störamplituden gestatten, deren Störamplitudenentwicklung frühzeitig zu verfolgen, indem diese Verfahren die im Experiment stets vorhandene Fehlerquellen wie Schwingungen der Meßsonden, Schaufelfrequenzen des Antriebes des Windkanals oder das Rauschen des Analogteils der Meßkette weitgehend ausschalten.

Eine für alle Experimente, die die Störungsentwicklung unter quasi natürlichen Bedin- 
gungen mit Resultaten numerischer Stabilitätsanalysen vergleichen, wichtige Voraussetzung an die vorhandenen natürlichen Störungen, nämlich deren spannweitige Kohärenz, wurde erstmals für die natürlich entstandenen instationären Störungen in dreidimensionalen Grenzschichten untersucht. Dabei zeigte sich die spannweitige Kohärenz über einen weiten Bereich als ausreichend groß, um die instationären Störungen mit dem in der Stabilitätstheorie gebräuchlichen Normalmodenansatz beschreiben zu können. Allerdings ist ein spannweitiger Abfall der Kohärenz deutlich vorhanden und wird in zukünftigen nichtlinearen Modellen Eingang finden müssen, da er insbesondere nichtlineare Wechselwirkungen abschwächt.

Es gelang im Experiment, Betrag und Richtung dominanter laufender Instabilitätswellen zu ermitteln. Dabei zeigte sich eine gute Übereinstimmung mit den nach linearer Theorie am meisten angefachten primären Instabilitäten der Grenzschicht. Der Vergleich zwischen der experimentell bestimmten Amplitudenentwicklung instationärer Störungen und der nach verschiedenen linearen Theorien vorhergesagten ergab bereits in einem frühen Stadium große Unterschiede. Zwar zeigte sich auch für instationäre Störungen ein stabilisierender Effekt der Oberflächenkrümmung und ein destabilisierender Effekt der Nichtparallelität; das gemessene Amplitudenwachstum blieb allerdings deutlich hinter den Vorhersagen zurück. Mutmaßliche Ursache dafür sind die bereits bei sehr kleinen Amplituden einsetzenden nichtlinearen Wechselwirkungen signifikanter Größe zwischen stationären Querströmungswirbeln und instationären Störungen. Inwieweit bereits existierende nichtlineare Theorien das vorgefundene Störungswachstum wiedergeben können, müssen konkrete Rechnungen zeigen.

Andere typisch nichtlineare Effekte von Störungswechselwirkungen in der Grenzschicht, wie die Grundströmungsverformung und die Sättigung stationärer oder instationärer Störungen, machen sich im vorliegenden Experiment erst in wesentlich späteren Stadien der Störungsentwicklung bemerkbar.

Ein Vergleich der beobachteten Sättigungsamplituden des vorliegenden Experiments mit denen eines in der Oberflächenrauhigkeit vergleichbaren Experimentes im selben Kanal an eine schiebenden Platte mit aufgeprägtem Druckgradient, ergaben bei Vorhandensein einer konvexen Oberflächenkrümmung kleinere stationäre und größere instationäre Sättigungsamplituden. Um eine klare kausale Zuordnung treffen zu können, fehlen allerdings auf nichtlinearen Theorien basierende Strömungsberechnungen bzw. Instabilitätsanalysen.

Der die laminare Grenzschichtentwicklung abschließende Prozeß des Zusammenbrechens konnte sowohl unter alleinigem Einfluß der Querströmungsinstabilität als auch in einer für technische Anwendung ebenso relevanten, wenngleich bisher unzureichend untersuchten Mischform unter Mitwirkung der Tollmien-Schlichting Instabilität beobachtet werden. Dabei zeigten sich qualitative Unterschiede in den durchlaufenen Szenarien. Für den ersten Fall wurde nach dem Sättigen stationärer und instationärer Störungen eine allmähliche Aufweitung des Störspektrums hin zu hohen Frequenzen beobachtet; es fand ein quasi allmählicher Übergang zur Turbulenz statt. In bisherigen Untersuchungen von ebenfalls durch Querströmungsinstabilität dominierten Umschlagsszenarien über ebenen Oberflächen wurden hochfrequente Geschwindigkeitsfluktuationen im unmittelbaren Vorfeld des 
Umschlags gefunden, die als sekundäre Instabilitäten einer primär gestörten Grenzschicht identifiziert werden konnten. Vermutlich resultiert das Fehlen dieser sekundären Instabilität aus den vergleichsweise kleinen stationären Sättigungsamplituden, eine mutmaßliche Folge der konvexen Oberflächenkrümmung.

Im Mischfall wurden hochfrequente Geschwindigkeitsfluktuationen im Vorfeld des dann relativ plötzlich einsetzenden Umschlags gefunden. Die Lage des gehäuften Auftretens dieser hochfrequenten Geschwindigkeitsfluktuationen bezüglich der stationären Querströmungswirbel ist signifikant verschieden von der Lage der bisher bekannten hochfrequenten sekundären Instabilität. Auch an dieser Stelle erhofft man sich, ausgehend von nichtlinearen Grenzschichtrechnungen und Instabilitätsanalysen einen tieferen Einblick in diese Prozesse. Insbesondere ist hier eine (sekundäre) Instabilitätsanalyse der primär gestörten Grundströmung notwendig, um eventuell ein Kriterium zu finden, an dem man die Einleitung des Umschlags aus Instabilitätsanalysen besser als bisher vorhersagen kann.

Zum Abschluss noch ein kurzer Blick auf die noch offenen Fragestellungen und ein Vorschlag für ein mögliches weiteres Vorgehen.

Nachdem durch die Ergebnisse des vorliegenden Experiments die Verhältnisse der natürlichen Störungsentwicklung weitgehend bekannt sind, kann man nun, mit Hilfe gezielter Anregung einzelner separater stationärer und instationärer Störungen, deren individuelle Entwicklung und gegenseitige Wechselwirkungen auch dort beobachten, wo Experimente unter natürlichen Bedingungen ihre größten Defizite haben - im Anfangsbreich der Störungsentwicklung mit seinen sehr kleinen Störungsamplituden, und im Bereich des Zusammenbruchs der laminaren Grenzschichtströmung mit seiner hohen Instationarität. In beiden Bereichen kann man durch bedingte Mittelungen der Meßsignale die Nutzsignalqualität deutlich verbessern. Desweiteren gelingt damit auch die Analyse der für die Ausbildung sekundärer Instabilitäten als wesentlich nachgewiesenen momentanen Grenzschichtprofile. Auch für diese Untersuchungen sind begleitende numerische Instabilitätsuntersuchungen sinnvoll, da man ihre Ergebnisse quantitativ mit der Entwicklung individueller Störungen im Experiment vergleichen kann.

Erst wenn man die gesamte Kette physikalischer Prozesse der Störungsentwicklung in der laminaren Grenzschicht, vom Einkoppeln von Störungen aus der Umgebung bis zum Zusammenbruch, richtig modelliert hat, ist ein weiterer wichtiger Schritt hin zu einem universellen Verfahren zur Strömungsberechnung getan: die Vorhersage der Lage des laminar turbulenten Übergangs. 


\section{Literaturverzeichnis}

[1] Reynolds, O. (1883) An experimental investigation of the circumstances which determine whether the motion of water shall be direct or sinous and of the law of resistance in parallel channels. Trans. Roy. Soc. London 174, S. 935ff oder scientific papers II, S. $51 f f$

[2] Rayleigh, Lord (1880) On the stability of certain fluid motions. scientific papers, 1, S. 474ff; 3, S. 17ff; 4,S. 203ff; 6,S. $197 f f$

[3] Tollmien, W. (1935) Ein allgemeines Kriterium der Instabilität laminarer Geschwindigkeitsverteilungen. Nach. Ges. Wiss. Göttingen, Math. Phys. Kl. Fachgr. 1, S. 79ff

[4] Gray,W.E. (1952) The effect of wing sweep on laminar flow. RAE TM Aero 256

[5] Gray,W.E. (1952) The nature of the boundary layer flow at the nose of a swept wing. RAE TM Aero 255

[6] Gregory,N. Stuart,J.T. Walker,W.S. (1955) On the stability of three dimensional boundary layers with application to the flow due to a rotating disc. Phil. Trans. R. Soc. Lond. A 248, S. 155ff

[7] Poll,D.I.A. (1985) Some observations of the transition process on the windward face of a long yawed cylinder. J. Fluid Mech. 150, S. 329ff

[8] Arnal,D. Coustols,E. Juillen,J.C. (1984) Experimental and theoretical study of transition phenomena on an infinite swept wing. Rech. Aerosp. No. 1984-4

[9] Michel,R. Arnal,D. Coustols,E. Juillen,J.C. (1984) Experimental and theoretical studies of boundary layer transition on a swept infinite wing. In Laminar-Turbulent transition, IUTAM Symp. Novosibirsk, UdSSR, 1984, (ed. V.V. Kozlov), S. 553ff, Springer Berlin Heidelberg

[10] Saric,W.S. Yeates,L.G. (1985) Experiments on the stability of cross flow vortices in swept wing flow. AIAA Pap. 86-0493

[11] Saric,W.S. (1998) Low speed boundary layer transition experiments. AIAA Educational Series, Oxford Press 
[12] Mack, L. (1984) Special Course on Stability and Transition of Laminar Flows. AGARD Report Nr. 709, Paper 3 „Boundary layer linear stability theory“

[13] Arnal,D. Juillen,J.C. (1987) Three dimensional transition studies at ONERA/CERT. AIAA Paper 87-1345

[14] Lerche, Th. (1997) Experimentelle Untersuchung nichtlinearer Strukturbildung im Transitionsprozeß einer instabilen dreidimensionalen Grenzschicht. Dissertation, Univ. Hannover, VDI-Fortschritt-Berichte Reihe 7, Nr. 310

[15] Deyhle,H ,Höhler,G ,Bippes, H. (1993) Experimental investigation of wave propagation in a three dimensional boundary layer flow. AIAA J. 31, 637

[16] Bippes,H. (1991) Experiments on transition in three-dimensional accelerated boundary-layer flows. Proc. RAS Boundary Layer Transition and Control, Cambridge, $U K, I S B N 0903409860$

[17] Herbert,T. Bertolotti, F.P. (1987) Stability analyses of nonparallel boundary layers. Bull. Am. Phys. Soc. 32, S. 2079ff

[18] Bertolotti,F.P. Herbert,T. Spalart,P.R. (1992) Linear and nonlinear stability of the Blasius boundary layer. J. Fluid Mech. 242, S. 441ff

[19] Lerche,Th. ,Bippes,H. (1996) Experimental investigations of crossflow instability under the influence of controlled disturbance excitation. in: Transitional Boundary Layers in Aeronautics, Coll. Royal Netherlands Academy of Arts and Sciences Amsterdam,1995 (eds. R.W.A.M. Henkes und J.L. Van Ingen), S. 137ff, North-Holland Press.

[20] Haynes, T.S. (1996) Nonlinear stability and saturation of crossflow vortices in swept wing boundary layers. PhD. Thesis, Arizona State Univ., Tempe, USA

[21] Collier, F.S.Jr. Malik, M.R. (1989) Curvature Effects on the stability of ThreeDimensional Laminar Boundary Layers. AGARD Conf. Proc. No. 438, S. 5,1

[22] Hein St. (1999), persönliche Mitteilungen

[23] Reibert,M.S. Saric,W.S. (1997) Review of swept wing transition. AIAA Paper 97-1816

[24] Reed, H.L. (1987) Wave interactions in swept wing flows. Phys. Fluids 30, S. 3419

[25] Fischer,T.M. Dallmann,U. (1991) Primary and secondary stability analysis of a threedimensional boundary-layer flow. Phys. Fluids A 3, S. 2378ff

[26] Meier,F. Kleiser,L. (1990) Numerical Simulation of transition due to cross-flow instability. in: Laminar-Turbulent Transition, IUTAM Symp. Toulouse, Frankreich, 1989, (eds. D. Arnal und R. Michel), S. 609ff, Springer. 
[27] Wagner, M. (1992) Numerische Untersuchungen zum laminar-turbulenten Übergang in zwei- und dreidimensionalen Grenzschichten. Dissertation zum Dr.-Ing., Univ. Karlsruhe, 1992 (auch DLR-FB 92-36, 1992)

[28] Wintergerste,T. Kleiser,L. (1996) Direct numerical simulation of transition in a threedimensional boundary layer. in: Transitional Boundary Layers in Aeronautics, Coll. Royal Netherlands Academy of Arts and Sciences Amsterdam, 1995 (eds. R.W.A.M. Henkes und J.L. Van Ingen), S. 145ff, North-Holland Press.

[29] Müller,W. Bestek,H. Fasel,H. (1995) Spatial direct numerical simulation of transition in a three-dimensional boundary layer. IUTAM Symp. Sendai, Japan, 1994 (ed. R. Kobayashi), S. 397ff, Springer.

[30] Malik,M.R. Li,F. Chang,C.-L. (1994) Cross-flow disturbances in three- dimensional boundary layers: Non-linear development, wave interaction, and secondary instability. J.Fluid Mech. 268, S. 1ff

[31] Bertolotti,F.P. (1995) On the birth and evolution of disturbances in three-dimensional boundary layers. In: Proc. IUTAM Symp. On Nonlinear Stability and Transition in Three-Dimensional Boundary Layers, Manchester, Großbritanien, 1994 (eds. P. W. Duck und P. Hall), S. 247ff, Kluwer Academic Publishers.

[32] Reibert,M.S. (1996) Nonlinear stability, saturation and transition in crossflowdominated boundary layers. PhD thesis, Arizona State Univ., Tempe, USA

[33] Kohama,Y. Saric,W.S. Hoos,J.A. (1991) A high-frequency, secondary instability of crossflow vortices, that leads to transition. Proc. R.A.S. Conf. On Boundary Layer Transition and Control, Cambridge, Großbritanien, S. 4,1ff

[34] Deyhle,H. Bippes,H. (1996) Disturbance growth in an unstable three- dimensional boundary layer and its dependence on environmental condition. J.Fluid Mech. 316, S. $73 f f$

[35] Nishioka,M. Asai,M. Iida,S. (1980) An experimental investigation of the secondary instability. IUTAM Symp. on Laminar - Turbulent Transition, Sendai, Japan, 1980.

[36] Fischer,T.M. Hein,St. Dallman,U. (1993) A theoretical approach for describing secondary stability features in three-dimensional boundary layer flows. AIAA Paper 93-0080

[37] Bippes,H. (1997) Basic experiments on transition in three-dimensional boundary layers dominated by crossflow instability.

[38] König, M. (1997) Persönliche Mitteilungen

[39] Eckelmann,H. (1997) Einführung in die Strömungsmeßtechnik Teubner, Stuttgart 
[40] Rosemann,H. (1989) Einfluß der Geometrie von Mehrfachhitzdrahtsonden auf die Ergebnisse in turbulenten Strömungen. Dissertation zum Dr.-Ing., Tech. Univ. Clausthal, 1989 (auch DLR-FB 89-26, 1989)

[41] Betchov,R. Criminale,W.O. (1967) Stability of Parallel Flows. Academic Press, New York

[42] Drazin,P.O. Reid,W.H. (1981) Hydrodynamic Stability. Cambridge University Press, Cambridge

[43] Bieler, H. (1986) Theoretische Untersuchungen über primäre Instabilitäten in dreidimensionalen Grenzschichtströmungen. DFVLR Forschungsbericht, DFVLR-FB 86-54

[44] Hein,St. Fischer,T.M. Dallmann,U. (1992) Untersuchungen zur sekundären Instabilität einer dreidimensionalen Grenzschichtströmung. DLR Institut für Strömungsmechanik, IB 221-92 A23

[45] Grosch,C.E. Salwen,H. (1978), The Continuous Spectrum of the Orr-Sommerfeld Equation. Part 1. The Spectrum and the Eigenfunctions. J. Fluid Mech. 87, S. 33ff

[46] Hein, St. (1998) persönliche Mitteilungen

[47] Deyhle, H. (1993) Einfluß der äußeren Strömungsbedingungen auf den Transitionsprozeß einer dreidimensionalen Grenzschicht. Dissertation, Univ. Hannover, VDIFortschritt-Berichte Reihe 7, Nr. 226

[48] Müller, B. (1990) Experimentelle Untersuchung der Querströmungsinstabilität im linearen und nichtlinearen Bereich des Transitionsgebietes. DLR Forschungsbericht, DLR-FB 90-09, 1990

[49] Bippes, H. (1995) Instabilities developing in the three-dimensional boundary layer on concave and convex curved surface. In Laminar-Turbulent Transition, IUTAM Symp. Sendai Japan, 1994 (ed. R. Kobayashi), S. 315ff. Springer

[50] Bippes, H. (1995) Experiments to the influence of surface curvature on the development of streamwise vortices in three-dimensional boundary-layer flows. Z. Flugwiss. Weltraumforsch. 19, S. 129ff.

[51] Fjørtoft, R. (1950) Application of integral theorems in deriving criteria of stability for laminar flows and the baroclinic circular vortex. Geofys. Publ. Oslo, Schweden, 17, No.6, S. 1ff.

[52] Wu, Y. (1999) persönliche Mitteilungen.

[53] Balachandarm, S.Streett, C.L. Malik, M.R. (1990) Secondary instability in rotating disk flows. AIAA 90-1527 
[54] Stolte, A. (1999) persönliche Mitteilungen.

[55] Crouch, J.D. (1993) Receptivity of three-dimensional boundary layers AIAA Paper 93-0074

[56] Malik, M.R. Balakumar,P. (1993) Linear Stability of Three-Dimensional Boundary Layers: Effect of Curvature an Non-Parallelism AIAA Paper 93-0079

[57] Hein, St. Bertolotti, F.P. Simen, M. Hanifi, A. Henningson, D. (1994) Linear nonlocal instability analysis - the linear NOLOT code. DLR-IB 223-94 A56

[58] Dalheimer, M. K. (1998) IATEX, kurz \& gut. O'Reilly, 1998, ISBN 3-89721-204-8

[59] Lüke, H.D. (1995) Signalübertragung: Grundlagen der digitalen und analogen Nachrichtenübertragungssysteme. Springer-Lehrbuch, 1995, ISBN 3-540-58753-5

[60] Lowes M. Paulik, A. (1995) Programmieren in C - ANSI Standard. B.G. Teubner Stuttgart, 1995, ISBN 3-519-22286-8

[61] Bronstein I.N. Semendjajew K.A. (1979) Taschenbuch der Mathematik. BSB Teubner Leipzig, und Verlag Nauka, Moskau, 1979

[62] Press W.H. Teukolsky, S.A. Vetterling, W.T. Flannery, B.P. (1994) Numerical Recipes in C (The Art of Scientific Computing) Cambridge Univ. Press, 2nd edition, 1994 
Anhang 



\section{Anhang A}

\section{Lineare Stabilitätsanalyse}

Die linerare Stabilitätsanalyse ist ein mathematisches Hilfsmittel zur Transitionsvorhersage in Grenzschichten. Dabei untersucht man die Stabilitätseigenschaften der Grenzschicht gegen diskrete, meist harmonische Störungen. Findet man die Störungen in Raum und/oder Zeit verstärkt, so stören sie mit wachsender Amplitude die sich ausbildende Grenzschicht. Überschreiten die Amplituden dabei einen bestimmten (empirisch bestimmten) Schwellenwert, so nimmt man Transition an.

Es gibt eine große Anzahl verschiedener Verfahren die Stabilitätsanalyse durchzuführen, aber alle gehen zunächst von den Grundgleichungen aus. Ich beschränke mich in dieser Darstellung auf viskose, nichtlineare Grenzschichtströmungen. Für diese lauten die NavierStokes-Gleichungen in kartesischen Koordinaten $(x, y, z)$

$$
\begin{aligned}
\frac{\partial U}{\partial t}+U \frac{\partial U}{\partial x}+V \frac{\partial U}{\partial y}+W \frac{\partial U}{\partial z} & =-\frac{1}{\rho} \frac{\partial p}{\partial x}+\nu \nabla^{2} U \\
\frac{\partial V}{\partial t}+U \frac{\partial V}{\partial x}+V \frac{\partial V}{\partial y}+W \frac{\partial V}{\partial z} & =-\frac{1}{\rho} \frac{\partial p}{\partial y}+\nu \nabla^{2} V \\
\frac{\partial W}{\partial t}+U \frac{\partial W}{\partial x}+V \frac{\partial W}{\partial y}+W \frac{\partial W}{\partial z} & =-\frac{1}{\rho} \frac{\partial p}{\partial z}+\nu \nabla^{2} W
\end{aligned}
$$

Die Kontinuitätsgleichung lautet

$$
\frac{\partial U}{\partial x}+\frac{\partial V}{\partial y}+\frac{\partial W}{\partial z}=0
$$

Man trennt für Geschwindigkeiten und Drucke den mittleren und zeitlich fluktuierenden Teil $Q(x, y, z, t)=q(x, y, z)+q^{\prime}(x, y, z, t) Q=u, v, w, p$, nimmt lokal Parallelströmung $(u=$ $u(y), v=0, w=w(y))$ an und setzt in A.1 und A.5 ein. Man erhält für den fluktuierenden 
Teil

$$
\begin{aligned}
\frac{\partial u^{\prime}}{\partial t}+u \frac{\partial u^{\prime}}{\partial x}+v \frac{\partial u^{\prime}}{\partial y}+w \frac{\partial u^{\prime}}{\partial z} & =-\frac{1}{\rho} \frac{\partial p^{\prime}}{\partial x}+\frac{1}{R e}\left(\frac{\partial^{2} u^{\prime}}{\partial x^{2}}+\frac{\partial^{2} u^{\prime}}{\partial y^{2}}+\frac{\partial^{2} u^{\prime}}{\partial z^{2}}\right) \\
\frac{\partial v^{\prime}}{\partial t}+u \frac{\partial v^{\prime}}{\partial x}+v \frac{\partial v^{\prime}}{\partial y}+w \frac{\partial v^{\prime}}{\partial z} & =-\frac{1}{\rho} \frac{\partial p^{\prime}}{\partial y}+\frac{1}{R e}\left(\frac{\partial^{2} v^{\prime}}{\partial x^{2}}+\frac{\partial^{2} v^{\prime}}{\partial y^{2}}+\frac{\partial^{2} v^{\prime}}{\partial z^{2}}\right) \\
\frac{\partial w^{\prime}}{\partial t}+u \frac{\partial w^{\prime}}{\partial x}+v \frac{\partial w^{\prime}}{\partial y}+w \frac{\partial w^{\prime}}{\partial z} & =-\frac{1}{\rho} \frac{\partial p^{\prime}}{\partial z}+\frac{1}{R e}\left(\frac{\partial^{2} w^{\prime}}{\partial x^{2}}+\frac{\partial^{2} w^{\prime}}{\partial y^{2}}+\frac{\partial^{2} w^{\prime}}{\partial z^{2}}\right)
\end{aligned}
$$

unter Vernachläßigung der fluktierender Größen quadratischer Ordnung. Durch Normierung aller Geschwindigkeiten mit der Geschwindigkeit am Grenzschichtrand $\left(U_{e}\right)$, aller Drucke mit dem Staudruck am Grenzschichtrand $\left(\rho_{e} * u_{e}^{2}\right)$ und aller Längen mit einer Grenzschichtdicke (z.B. $L=\delta_{99 \%}$ ) genügt ab hier die Betrachtung dimensionsloser Größen. Die Reynoldszahl wurde mit $R e=u_{e} * L / \nu$ gebildet.

Wir betrachten harmonischer Störungen der Grenzschicht der Form

$$
\left(u^{\prime}, v^{\prime}, w^{\prime}, p^{\prime}\right)=[f(y), g(y), h(y), \Pi(y)] \exp i(\alpha x+\beta z-\omega t),
$$

mit $\left(\alpha=2 \pi L / \lambda_{x}, \beta=2 \pi L / \lambda_{z}, \omega=2 \pi f L / u_{e} ; f, \lambda_{x}, \lambda_{z}, \omega \epsilon C\right)$. also ebener Wellen bestimmter Richtung und komplexer Amplitude. Setzen wir diesen Ansatz in Gleichung A.6 ein, erhalten wir

$$
\begin{aligned}
i(\alpha u+\beta w-\omega) f+u^{\prime} g & =-i \alpha \Pi+\frac{1}{R e}\left[f^{\prime \prime}-\left(\alpha^{2}+\beta^{2}\right) f\right] \\
i(\alpha u+\beta w-\omega) g & =-\Pi^{\prime}+\frac{1}{R e}\left[g^{\prime \prime}-\left(\alpha^{2}+\beta^{2}\right) g\right] \\
i(\alpha u+\beta w-\omega) h+w^{\prime} g & =-i \beta \Pi+\frac{1}{R e}\left[h^{\prime \prime}-\left(\alpha^{2}+\beta^{2}\right) h\right] \\
i(\alpha f+\beta h)+g^{\prime} & =0,
\end{aligned}
$$

mit ${ }^{\prime}=d / d y$ und $"=d^{2} / d y^{2}$. Wir eleminieren aus dieser Gleichung $\Pi,(\alpha f+\beta h)$ und $\left(\alpha f^{\prime}+\beta h^{\prime}\right)$ und erhalten eine homogene, lineare Differentialgleichung vierter Ordnung,

$$
\begin{aligned}
& g^{\prime \prime \prime \prime}-2\left(\alpha^{2}+\beta^{2}\right) g^{\prime \prime}+\left(\alpha^{2}+\beta^{2}\right)^{2} g \\
= & i \operatorname{Re}\left\{(\alpha u+\beta w-\omega)\left[g^{\prime \prime}-\left(\alpha^{2}+\beta^{2}\right) g\right]-\left(\alpha u^{\prime \prime}+\beta w^{\prime \prime}\right) g\right\},
\end{aligned}
$$

die Orr-Sommerfeld-Gleichung. Es gelten die Randbedingungen am Grenzschichtrand

$$
\begin{aligned}
y=0 & : g(0)==g^{\prime}(0)==0 \text { (Haftbedingung) } \\
y \rightarrow \infty & : g(\infty)==g^{\prime}(\infty)==0 \text { (endliche Energie in der Störung). }
\end{aligned}
$$

Für die weiteren Betrachtungen ist es sinnvoll, in Gleichung

$$
\begin{array}{ll}
Z_{1}=\alpha f+\beta h & Z_{2}=Z_{1}^{\prime} \\
Z_{3}=g & Z_{4}=P i \\
Z_{5}=\alpha h-\beta f & Z_{6}=Z_{5}^{\prime}
\end{array}
$$


zu ersetzen. Man erhält

$$
\begin{aligned}
Z_{1}^{\prime}= & Z_{2} \\
Z_{2}^{\prime}= & {\left[\alpha^{2}+\beta^{2}+i \operatorname{Re}(\alpha u+\beta w-\omega)\right] Z_{1}+\left(\alpha u^{\prime}+\beta w^{\prime}\right) \operatorname{Re} Z_{3} } \\
& +i\left(\alpha^{2}+\beta^{2}\right) \operatorname{Re} Z_{4} \\
Z_{3}^{\prime}= & -i Z_{1} \\
Z_{4}^{\prime}= & -\frac{i}{\operatorname{Re}} Z_{2}-\left[i(\alpha u+\beta w-\omega)+\frac{\alpha^{2}+\beta^{2}}{\operatorname{Re}] Z_{3}}\right. \\
Z_{5}^{\prime}= & Z_{6} \\
Z_{6}^{\prime}= & \left(\alpha w^{\prime}-\beta u^{\prime}\right) \operatorname{Re} Z_{3}+\left[\alpha^{2}+\beta^{2}+i \operatorname{Re}(\alpha u+\beta w-\omega)\right] Z_{5},
\end{aligned}
$$

mit den Randbedingungen

$$
\begin{aligned}
y=0: & Z_{1}(0)=Z_{3}(0)=0 \\
& Z_{1}(y) \rightarrow 0 \\
y \rightarrow \infty: & Z_{3}(y) \rightarrow 0 \\
& Z_{5}(y) \rightarrow 0
\end{aligned}
$$

Glücklicherweise entkoppeln die letzten beiden Gleichungen aus System A.19. Es ist praktisch, das verbleibende System als Matrix zu schreiben.

$$
\vec{Z}^{\prime}=A \vec{Z}
$$

mit $\vec{Z}=\left(Z_{1}, Z_{2}, Z_{3}, Z_{4}\right), \vec{Z}^{\prime}=\left(d Z_{1} / d y, d Z_{2} / d y, d Z_{3} / d y, d Z_{4} / d y\right)$ und

$$
A=\left(\begin{array}{cccc}
0 & 1 & 0 & 0 \\
a & 0 & b & c \\
-i & 0 & 0 & 0 \\
0 & -i / R e & d & 0
\end{array}\right)
$$

mit

$$
\begin{aligned}
a & =\alpha^{2}+\beta^{2}+i \operatorname{Re}(\alpha u+\beta w-w) \\
b & =\left(\alpha u^{\prime}+\beta w^{\prime}\right) \operatorname{Re} \\
c & =i \operatorname{Re}\left(\alpha^{2}+\beta^{2}\right) \\
d & =-\left[i(\alpha u+\beta w-\omega)+\frac{\alpha^{2}+\beta^{2}}{\operatorname{Re}}\right]
\end{aligned}
$$

Zur Lösung des hier hergeleiteten Differentialgleichungssystems bieten sich 2 Verfahren an. 
- zeitliche Theorie: $\alpha, \beta \epsilon R ; \omega \epsilon C$

- räumliche Theorie: $\alpha, \beta \epsilon C ; \omega \epsilon R$

Die zeitliche Theorie gibt das Störungswachstum mit fortlaufender Zeit an, wogegen die räumliche Theorie das räumliche Anwachsen angibt. Beide Verfahren sind für konvektive Instabilitäten ineinander überführbar. Konvektive Instabilitäten weisen einen von Null verschiedenen Betrag der Gruppengeschwindigkeit der Störungen aus.

Die Stabilitätsgleichungen löst man numerisch. Dazu existieren mehrere Verfahren. Insbesondere Spektralverfahren tun dies schnell und genau. Alternativ kann man auch shooting-Verfahren nutzen, die den Vorteil haben, leicht mit numerischen Standardmethoden implementierbar zu sein.

Dazu schaut man die Stabilitätsgleichungen am Grenzschichtrand an und findet die Koeffizienten von A konstant. Dort existieren zwei leicht zu findende analytische Lösungen (Eigenwertproblem). Diese Lösungen integriert man numerisch zur Wand hin und versucht die noch freien Parameter mittel eines Newton- Verfahrens so zu wählen, daß man an der Wand die Haftbedingungen erfüllt.

Als Lösungen ergeben sich Paare $(\alpha, \beta, \omega)$, je nach Verfahren mit reelem $\alpha, \beta$ und komplexem $\omega$ oder umgekehrt. Der Imaginärteil gibt dann die zeitliche oder räumliche Anfachung zu Störungen der Wellenzahl $(\alpha, \beta)$ und der Frequenz (Rew) an. 
Anhang B

Profilkoordinaten HQ26 


\begin{tabular}{|c|c|c|c|c|c|c|c|c|}
\hline Nr. & $\mathrm{x} / \mathrm{c}$ & $\mathrm{z} / \mathrm{c}$ & Nr. & $\mathrm{x} / \mathrm{c}$ & $\mathrm{z} / \mathrm{c}$ & Nr. & $\mathrm{x} / \mathrm{c}$ & $\mathrm{z} / \mathrm{c}$ \\
\hline 1 & 0,99998 & 0,0005 & 22 & 0,67049 & 0,05741 & 43 & 0,20918 & 0,10939 \\
\hline 2 & 0,99907 & 0,00051 & 23 & 0,64549 & 0,06371 & 44 & 0,19131 & 0,10571 \\
\hline 3 & 0,99634 & 0,00051 & 24 & 0,62045 & 0,07013 & 45 & 0,17402 & 0,10166 \\
\hline 4 & 0,9918 & 0,00054 & 25 & 0,59547 & 0,0766 & 46 & 0,15733 & 0,09728 \\
\hline 5 & 0,98548 & 0,0007 & 26 & 0,57068 & 0,08305 & 47 & 0,1413 & 0,09257 \\
\hline 6 & 0,97737 & 0,00103 & 27 & 0,54619 & 0,08941 & 48 & 0,12597 & 0,08759 \\
\hline 7 & 0,96751 & 0,00158 & 28 & 0,5221 & 0,0956 & 49 & 0,11137 & 0,08235 \\
\hline 8 & 0,95593 & 0,00245 & 29 & 0,49855 & 0,10148 & 50 & 0,09754 & 0,07691 \\
\hline 9 & 0,94272 & 0,00368 & 30 & 0,47566 & 0,10688 & 51 & 0,08452 & 0,07127 \\
\hline 10 & 0,92798 & 0,00534 & 31 & 0,45341 & 0,11148 & 52 & 0,07233 & 0,06549 \\
\hline 11 & 0,91182 & 0,00747 & 32 & 0,43158 & 0,11515 & 53 & 0,06102 & 0,05959 \\
\hline 12 & 0,89436 & 0,01005 & 33 & 0,40999 & 0,11794 & 54 & 0,05059 & 0,0536 \\
\hline 13 & 0,87571 & 0,01307 & 34 & 0,38861 & 0,11993 & 55 & 0,04109 & 0,04758 \\
\hline 14 & 0,85595 & 0,01652 & 35 & 0,36746 & 0,12119 & 56 & 0,03253 & 0,04155 \\
\hline 15 & 0,83519 & 0,02039 & 36 & 0,34653 & 0,12175 & 57 & 0,02493 & 0,03554 \\
\hline 16 & 0,81352 & 0,02467 & 37 & 0,32586 & 0,12167 & 58 & 0,01831 & 0,02964 \\
\hline 17 & 0,79106 & 0,02933 & 38 & 0,30548 & 0,12096 & 59 & 0,01269 & 0,02386 \\
\hline 18 & 0,7679 & 0,03435 & 39 & 0,28543 & 0,11968 & 60 & 0,00808 & 0,01825 \\
\hline 19 & 0,74416 & 0,03971 & 40 & 0,26573 & 0,11784 & 61 & 0,00449 & 0,0129 \\
\hline 20 & 0,71994 & 0,04537 & 41 & 0,24644 & 0,11548 & 62 & 0,00193 & 0,00787 \\
\hline \multirow[t]{2}{*}{21} & 0,69534 & 0,05129 & 42 & 0,22757 & 0,11266 & 63 & 0,00043 & 0,00327 \\
\hline & & & & & & 64 & 0 & 0 \\
\hline
\end{tabular}

Tabelle B.1: Profilkoordinaten Oberseite, $c=0,65 \mathrm{~m}$ 


\begin{tabular}{||c|c|c||c|c|c||c|c|c||}
\hline Nr. & $\mathrm{x} / \mathrm{c}$ & $\mathrm{z} / \mathrm{c}$ & $\mathrm{Nr}$. & $\mathrm{x} / \mathrm{c}$ & $\mathrm{z} / \mathrm{c}$ & $\mathrm{Nr}$. & $\mathrm{x} / \mathrm{c}$ & $\mathrm{z} / \mathrm{c}$ \\
\hline 65 & $2 \mathrm{e}-07$ & $-0,00077$ & 84 & 0,25792 & $-0,02666$ & 103 & 0,75138 & $-0,01926$ \\
\hline 66 & 0,00099 & $-0,00393$ & 85 & 0,28217 & $-0,02677$ & 104 & 0,77429 & $-0,0184$ \\
\hline 67 & 0,00372 & $-0,00647$ & 86 & 0,30705 & $-0,0268$ & 105 & 0,79637 & $-0,01749$ \\
\hline 68 & 0,00819 & $-0,00888$ & 87 & 0,33251 & $-0,02677$ & 106 & 0,81757 & $-0,01654$ \\
\hline 69 & 0,0142 & $-0,01113$ & 88 & 0,35845 & $-0,02666$ & 107 & 0,83782 & $-0,01555$ \\
\hline 70 & 0,02169 & $-0,0132$ & 89 & 0,38481 & $-0,0265$ & 108 & 0,85706 & $-0,01452$ \\
\hline 71 & 0,03061 & $-0,01512$ & 90 & 0,4115 & $-0,0263$ & 109 & 0,87524 & $-0,01343$ \\
\hline 72 & 0,04091 & $-0,01689$ & 91 & 0,43842 & $-0,02604$ & 110 & 0,8923 & $-0,01228$ \\
\hline 73 & 0,05255 & $-0,01848$ & 92 & 0,46552 & $-0,02573$ & 111 & 0,90822 & $-0,01104$ \\
\hline 74 & 0,06552 & $-0,01989$ & 93 & 0,49269 & $-0,02538$ & 112 & 0,92297 & $-0,00975$ \\
\hline 75 & 0,07978 & $-0,02115$ & 94 & 0,51987 & $-0,02498$ & 113 & 0,93654 & $-0,00839$ \\
\hline 76 & 0,09528 & $-0,02227$ & 95 & 0,54695 & $-0,02453$ & 114 & 0,9489 & $-0,00699$ \\
\hline 77 & 0,11198 & $-0,02323$ & 96 & 0,57388 & $-0,02405$ & 115 & 0,96004 & $-0,00558$ \\
\hline 78 & 0,12983 & $-0,02405$ & 97 & 0,60057 & $-0,0235$ & 116 & 0,97 & $-0,00417$ \\
\hline 79 & 0,14878 & $-0,02475$ & 98 & 0,62694 & $-0,02291$ & 117 & 0,97876 & $-0,00294$ \\
\hline 80 & 0,16878 & $-0,02534$ & 99 & 0,65291 & $-0,02227$ & 116 & 0,98616 & $-0,00196$ \\
\hline 81 & 0,18976 & $-0,02581$ & 100 & 0,67842 & $-0,02159$ & 119 & 0,99209 & $-0,00125$ \\
\hline 82 & 0,21165 & $-0,0262$ & 101 & 0,70338 & $-0,02085$ & 120 & 0,99643 & $-0,0008$ \\
\hline 83 & 0,2344 & $-0,02647$ & 102 & 0,72773 & $-0,02007$ & 121 & 0,9991 & $-0,00057$ \\
\hline & & & & & & 122 & 1 & $-0,0005$ \\
\hline
\end{tabular}

Tabelle B.2: Profilkoordinaten Unterseite, $\mathrm{c}=0,65 \mathrm{~m}$ 


\section{Anhang C}

\section{Druckverteilung}

Die hier angegebene Druckverteilung diente zur Grundströmungsrekonstruktion für die numerischen Untersuchungen zum vorliegenden Experiment.

- $U_{\infty}=28,07 \mathrm{~m} / \mathrm{s}$

- $p_{0}=469 \mathrm{~Pa}$
- $\operatorname{Re}=1,198 \cdot 10^{6}$

- Anstellwinkel: $-3,53^{\circ}$ 


\begin{tabular}{||c|c||c|c||}
\hline $10000 \cdot \mathrm{x} / \mathrm{c}$ & $p / p_{0}$ & $10000 \cdot \mathrm{x} / \mathrm{c}$ & $p / p_{0}$ \\
\hline 9820 & 0,035409 & 4182 & $-0,18419$ \\
\hline 9626 & 0,015581 & 3609 & $-0,2038$ \\
\hline 9419 & $-0,0050995$ & 3072 & $-0,23451$ \\
\hline 9220 & $-0,033455$ & 2620 & $-0,25988$ \\
\hline 9003 & $-0,043476$ & 2171 & $-0,30465$ \\
\hline 8769 & $-0,05371$ & 1780 & $-0,35326$ \\
\hline 8549 & $-0,057547$ & 1479 & $-0,4266$ \\
\hline 8308 & $-0,066288$ & 1160 & $-0,48992$ \\
\hline 7923 & $-0,076522$ & 931 & $-0,55324$ \\
\hline 7512 & $-0,087822$ & 783 & $-0,64481$ \\
\hline 7040 & $-0,096989$ & 585 & $-0,73638$ \\
\hline 6585 & $-0,10808$ & 414 & $-0,89437$ \\
\hline 6034 & $-0,12364$ & 274 & $-1,0796$ \\
\hline 5415 & $-0,13195$ & 157 & $-1,464$ \\
\hline 4803 & $-0,15669$ & 51 & $-2,2674$ \\
\hline
\end{tabular}

Tabelle C.1: Druckverteilung Unterseite 


\begin{tabular}{||c|c||c|c||}
\hline $10000 \cdot \mathrm{x} / \mathrm{c}$ & $p / p_{0}$ & $10000 \cdot \mathrm{x} / \mathrm{c}$ & $p / p_{0}$ \\
\hline 0 & $-1,8299$ & 4271 & $-0,39014$ \\
\hline 22 & 0,10044 & 4443 & $-0,3684$ \\
\hline 74 & 0,44625 & 4622 & $-0,34345$ \\
\hline 97 & 0,4923 & 4777 & $-0,33215$ \\
\hline 251 & 0,47226 & 4919 & $-0,32512$ \\
\hline 403 & 0,38016 & 5082 & $-0,30358$ \\
\hline 571 & 0,30554 & 5242 & $-0,22216$ \\
\hline 763 & 0,20661 & 5397 & $-0,16208$ \\
\hline 934 & 0,12581 & 5555 & $-0,14085$ \\
\hline 1155 & 0,0401 & 5786 & $-0,11852$ \\
\hline 1454 & $-0,052217$ & 6111 & $-0,085754$ \\
\hline 1786 & $-0,1456$ & 6502 & $-0,054242$ \\
\hline 2166 & $-0,20252$ & 7039 & $-0,016378$ \\
\hline 2617 & $-0,32853$ & 7514 & 0,012639 \\
\hline 3003 & $-0,39782$ & 7954 & 0,047007 \\
\hline 3322 & $-0,41338$ & 8362 & 0,059224 \\
\hline 3532 & $-0,41679$ & 8759 & 0,074617 \\
\hline 3772 & $-0,42447$ & 9098 & 0,08662 \\
\hline 3935 & $-0,42937$ & 9399 & 0,092142 \\
\hline 4109 & $-0,41232$ & 9663 & 0,085064 \\
\hline 4271 & $-0,39014$ & & \\
\hline & & & \\
\hline
\end{tabular}

Tabelle C.2: Druckverteilung Oberseite 


\section{Danksagung}

Die vorliegende Arbeit entstand während meiner Tätigkeit als Doktorand am Institut für Strömungsmechanik des Deutschen Zentrums für Luft- und Raumfahrt e.V. (DLR) in Göttingen. Sie war Teil des Graduiertenkollegs „Strömungsinstabilitäten und Turbulenz" der Universität Göttingen und wurde von diesem mit einem Stipendium unterstützt.

Mein besonderer Dank gehört Herrn Dr. H. Bippes, der das Thema der Arbeit anregte und sie betreute. Sein Vertrauen in meine Arbeit ermöglichte mir eine selbständige Tätigkeit und die vielen anregenden Diskussionen mit ihm trugen maßgeblich zur Qualität der vorliegenden Arbeit bei.

Ich danke Herrn Professor Dr. H. Eckelmann, dem Hauptreferenten der Arbeit und Sprecher des Graduiertenkollegs, und Herrn Prof. Dr. D. Ronneberger, dem Korreferenten. Sie begleiteten die Arbeit mit Interesse und zahlreichen Anregungen.

Mit fruchtbaren Diskussionen begleiteten die Mitglieder der Abteilung Transition und Turbulenz, unter Leitung von Herrn Dr. U. Dallmann, das Vorankommen der Arbeit. Insbesondere gilt hier mein Dank Herrn Dipl.-Ing. Stefan Hein für die gute Zusammenarbeit bei den begleitenden Rechnungen zum Experiment und Herrn H. Mattner für die Unterstützung bei der Durchführung der Experimente. Seine große Erfahrung bei der Durchführung von Experimenten und sein hohes persönliches Engagement trugen wesentlich zum guten Gelingen der Experimente bei.

Ich danke den Mitarbeitern des Niedergeschwindigkeitswindkanals Braunschweig unter Leitung von Herrn Dr. Otto für die gute Zusammenarbeit.

Meinen Eltern, Petra und Bernd Pöthke, gilt ein besonderer Dank für ihren Beistand, ihr Vertrauen und die Unterstützung während der gesamten Zeit meiner Ausbildung. Frau Dr. A. Beige danke ich für die gute Zusammenarbeit während der Zeit unseres Studiums.

Göttingen, im September 1999. 
